Documents de travail du Département des Affaires économiques de l'OCDE No. 1455

\title{
France: Améliorer l'efficience du système de santé
} Antoine Goujard 
Organisation de Coopération et de Développement Économiques

Organisation for Economic Co-operation and Development

02-Jan-2018

DEPARTEMENT DES AFFAIRES ECONOMIQUES

Français - Or. Anglais

FRANCE: AMÉLIORER L'EFFICIENCE DU SYSTÈME DE SANTÉ

DÉPARTEMENT DES AFFAIRES ÉCONOMIQUES DOCUMENTS DE TRAVAIL No. 1455

\section{Par Antoine Goujard}

Les documents de travail de l'OCDE ne doivent pas être présentés comme exprimant les vues officielles de l'OCDE ou de ses pays membres. Les opinions exprimées et les arguments employés sont ceux des auteur(s).

Accord pour publication donné par Álvaro Pereira, Directeur, Branche des études nationales, Département des Affaires économiques.

Les Documents de travail du Département des Affaires économiques sont disponibles à www.oecd.org/eco/workingpapers

JT03425123

Ce document, ainsi que les données et cartes qu'il peut comprendre, sont sans préjudice du statut de tout territoire, de la souverain eté s'exerçant sur ce dernier, du tracé des frontières et limites internationales, et du nom de tout territoire, ville ou région. 
Les documents de travail de l'OCDE ne doivent pas être présentés comme exprimant les vues officielles de l'OCDE ou de ses pays membres. Les opinions exprimées et les arguments employés sont ceux des auteur(s).

Les documents de travail exposent des résultats préliminaires ou des travaux de recherche en cours menés par l'auteur/les auteurs et sont publiés pour stimuler le débat sur un large éventail de questions sur lesquelles l'OCDE travaille.

Les commentaires sur les documents de travail sont les bienvenus et peuvent être adressés au Département des Affaires économiques, OCDE, 2 rue André-Pascal, 75775 Paris Cedex 16, France ou à l'adresse mél. suivante : eco.contact@oecd.org.

Les Documents de travail du Département des Affaires économiques sont disponibles à www.oecd.org/eco/workingpapers.

Les données statistiques concernant Israël sont fournies par et sous la responsabilité des autorités israéliennes compétentes. L'utilisation de ces données par l'OCDE est sans préjudice du statut des hauteurs du Golan, de Jérusalem Est et des colonies de peuplement israéliennes en Cisjordanie aux termes du droit international.

\section{(C) OCDE (2018)}

Vous êtes autorisés à copier, télécharger ou imprimer du contenu OCDE pour votre utilisation personnelle. Vous pouvez inclure des extraits des publications, des bases de données et produits multimédia de l'OCDE dans vos documents, présentations, blogs, sites Internet et matériel d'enseignement, sous réserve de faire mention de la source OCDE et du copyright. Les demandes pour usage commercial ou de traduction devront être adressées à rights@oecd.org. 


\section{ABSTRACT/RÉSUMÉ \\ France: Améliorer l'efficience du système de santé}

Le système de santé français offre des soins de haute qualité. Les résultats moyens sur le plan de la santé sont bons, le public est très satisfait du système de santé et les restes à charge sont en moyenne faibles. Comme dans d'autres pays de l'OCDE, le progrès technologique accroît l'espérance et la qualité de vie, alors que la population vieillissante nécessite des services toujours plus nombreux et diversifiés. Les principales difficultés consistent à inciter les professionnels de la santé à adopter un comportement efficient, à enrayer la hausse des dépenses pharmaceutiques, à renforcer le rôle des assureurs en tant qu'agents acheteurs et à assurer la maîtrise des coûts. Une information de bonne qualité et des mécanismes de financement appropriés renforceraient les incitations à l'efficience. Les différences de couverture selon les maladies et les groupes sociaux montrent la nécessité de prêter davantage attention à la coordination entre l'assurance maladie obligatoire et l'assurance complémentaire. Les réformes en cours visant à améliorer la coordination entre les professionnels de santé et à renforcer le rôle de la prévention vont dans le bon sens. Cependant, le développement de mécanismes de rémunération à la capitation qui permettent d'avoir moins d'incitations à multiplier les actes et d'encourager les professionnels de santé à s'occuper plus longtemps des patients, tout comme les paiements en fonction des résultats dans les soins primaires doivent être renforcés afin de faire face à la prévalence croissante des maladies chroniques et de réduire la demande induite par l'offre et les disparités sociales en terme d'accès aux soins.

Ce Document de travail se rapporte à l'Étude économique de l'OCDE de la France 2017

(http://www.oecd.org/fr/economie/etude-economique-france.htm)

Classification JEL : I1 1; I12; I13; I15; I18

Mots clefs: France, système de santé, politique de santé, coordination du système de santé, disparités de santé, vieillissement, hôpital, soins primaires de santé, dépenses pharmaceutiques, génériques, prévention, assurances médicales, tarification à l'acte, démographie médicale, professions médicales.

$* * * * * * * * * *$

\section{France: Improving the efficiency of the health-care system}

France's health-care system offers high-quality care. Average health outcomes are good, public satisfaction with the health-care system is high, and average household out-of-pocket expenditures are low. As in other OECD countries, technology is expanding possibilities for life extension and quality, and spending is rising steadily, while an ageing population requires substantially more and different services. The main challenges are to promote prevention and cost-efficient behaviour by care providers, tackle the high spending on pharmaceuticals, strengthen the role of health insurers as purchasing agents and secure cost containment. Good-quality information and appropriate financing schemes would ensure stronger efficiency incentives. Disparities of coverage across social groups and health services suggest paying greater attention to co-ordination between statutory and complementary insurance provision. Ongoing reforms to improve prevention and co-ordination among care providers are steps in the right direction. However, progress in the development of capitation-based payment schemes, which can reduce the incentives to increase the number of medical acts and encourage health professionals to spend more time with their patients, and performance-based payment schemes in primary care need to be stepped up to respond to the increasing prevalence of chronic diseases and curb supplier-induced demand and social disparities in access to care.

This Working Paper relates to the 2017 OECD Economic Survey of France

(www.oecd.org/eco/surveys/economic-survey-france.htm).

JEL classification: I11; I12; I13; I15; I18

Keywords: France, health care system, health policy, healthcare coordination, health disparities, ageing, hospital, primary healthcare, pharmaceutical expenditures, generics, prevention, health insurance, fee-for-services, medical demography, health practitioners. 


\section{TABLE DES MATIÈRES}

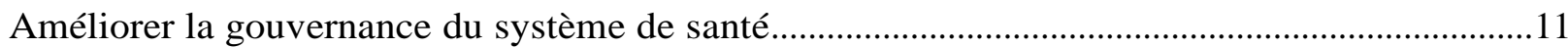

Améliorer le financement des dépenses de santé......................................................................11

Réduire les différences de couverture et renforcer les incitations visant les professionnels

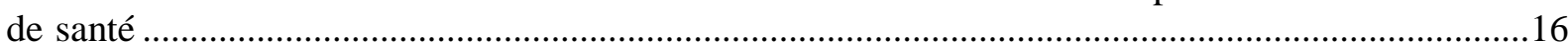

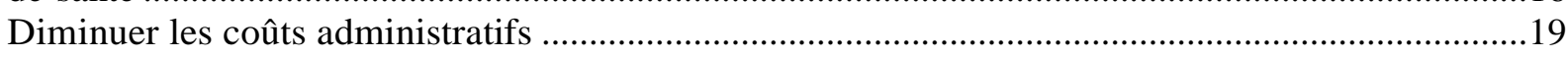

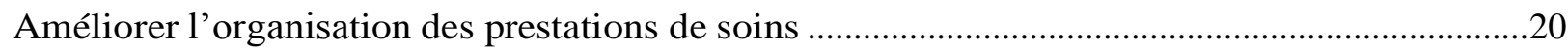

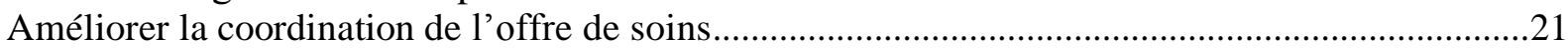

Adapter le réseau de soins primaires et son système de rémunération .........................................25

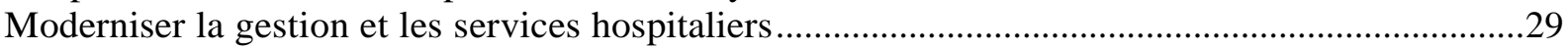

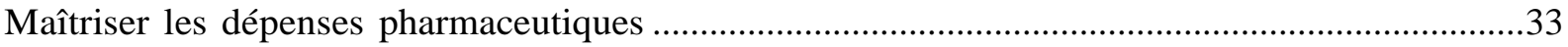

Investir dans la prévention et adopter des styles de vie plus sains ................................................37

Améliorer la prévention et lutter contre les comportements à risque ..............................................37

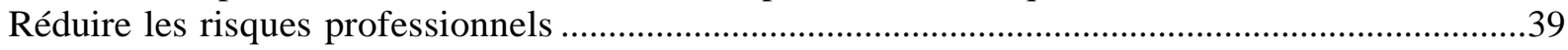

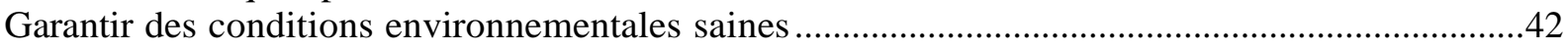

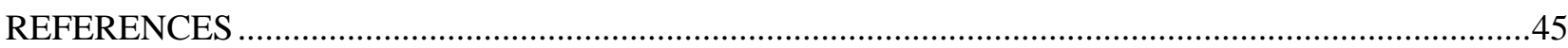

\section{Tableau}

1. Hausse prévue des dépenses publiques de santé en France, 2010-60 .........................................7

2. Structure du financement des différents produits et services médicaux, 2006-15 ........................16

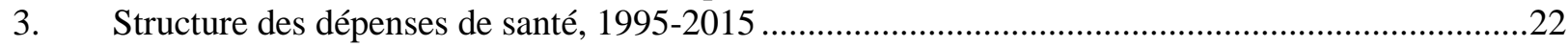

\section{Figures}

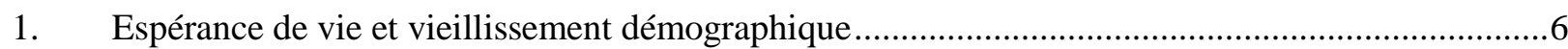

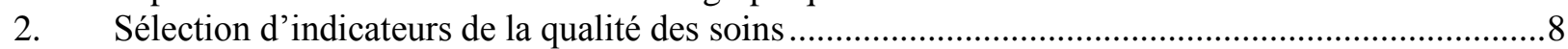

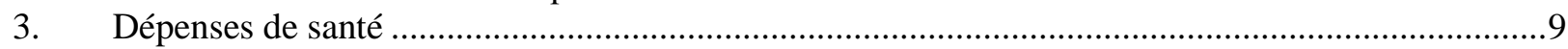

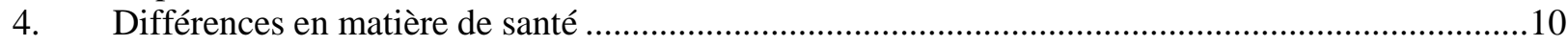

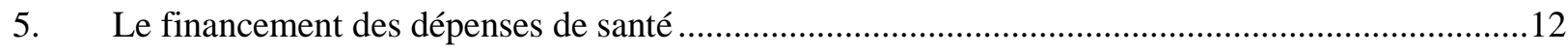

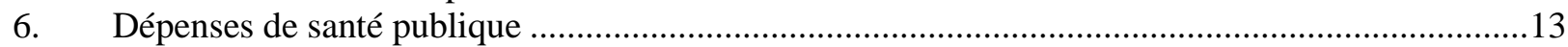

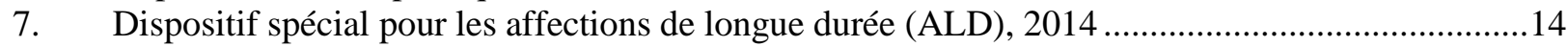

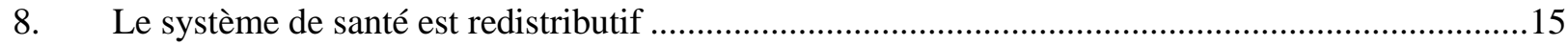

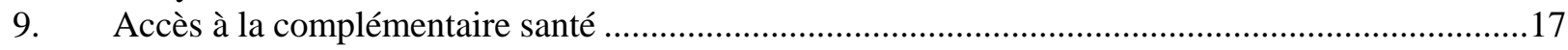

10. Accès aux régimes complémentaires d'assurance maladie par groupes d'âge .............................19

11. Les dépenses administratives au titre de la santé sont élevées....................................................20

12. Sélection d'indicateurs de prévention et de coordination entre les prestataires de soins ..................23

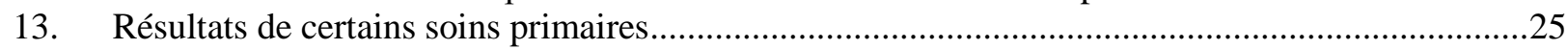

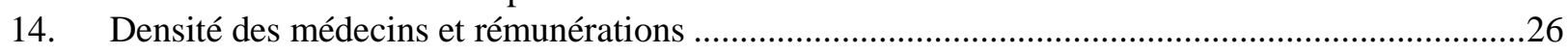

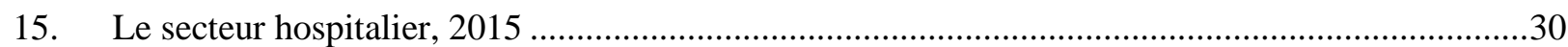

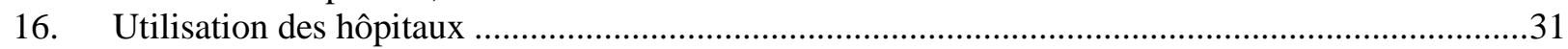

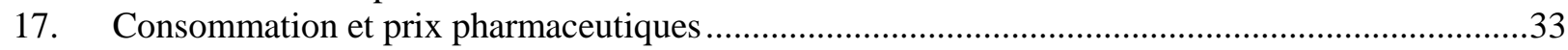

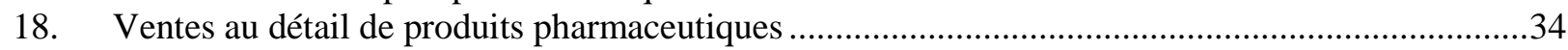

19. Consommation de génériques ..............................................................................................

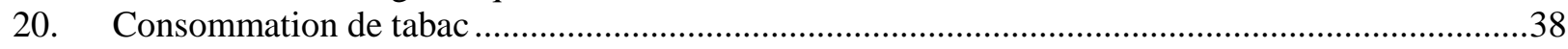

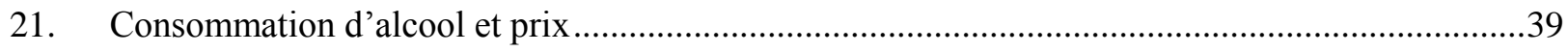

22. Prévalence de l'obésité et régime alimentaire ..............................................................................40 


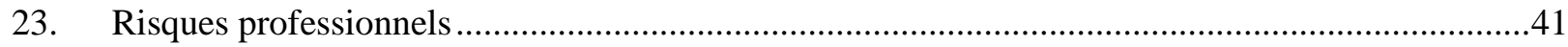

24. Congés maladie et jours d'absence liés aux accidents du travail et maladies professionnelles ......42

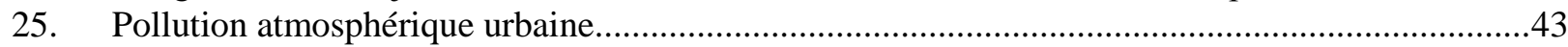

\section{Encadrés}

Encadré 1. Demande future de soins médicaux et de longue durée. 7

Encadré 2. Prévision des dépenses de santé publique et des objectifs annuels de dépenses (ONDAM)...12 Encadré 3. Paiement des hôpitaux et système des groupes homogènes de séjours (GHS) .........................30

Recommandations pour améliorer l'efficience du système de santé .......................................................44 


\title{
France : Améliorer l'efficience du système de santé
}

\author{
Par Antoine Goujard ${ }^{1}$
}

Les résultats de la France en termes de santé sont bons. D'après différentes mesures, l'espérance de vie de la population française, des femmes notamment, est parmi les plus élevées de l'OCDE (graphique 1, parties A et B), et le pays affiche l'une des meilleures espérances de vie en bonne santé de l'Union européenne (OMS, 2016). En outre, ces indicateurs s'améliorent régulièrement (Blanpain, 2016 et partie C). Toutefois, le système français va être soumis à des pressions croissantes du fait de l'accélération du vieillissement démographique prévue à compter du milieu des années 2020, et de la forte hausse de la part des plus de 80 ans - principaux bénéficiaires, par tête, des soins de santé et des soins de longue durée (parties C et D; Albouy et al., 2009). L'évolution des prix et le progrès technique risquent également de tirer les dépenses de santé vers le haut (Fall et al., 2014). Les simulations macro- et microéconomiques mettent en évidence des hausses considérables des dépenses de santé jusqu'en

\section{Graphique 1. Espérance de vie et vieillissement démographique}

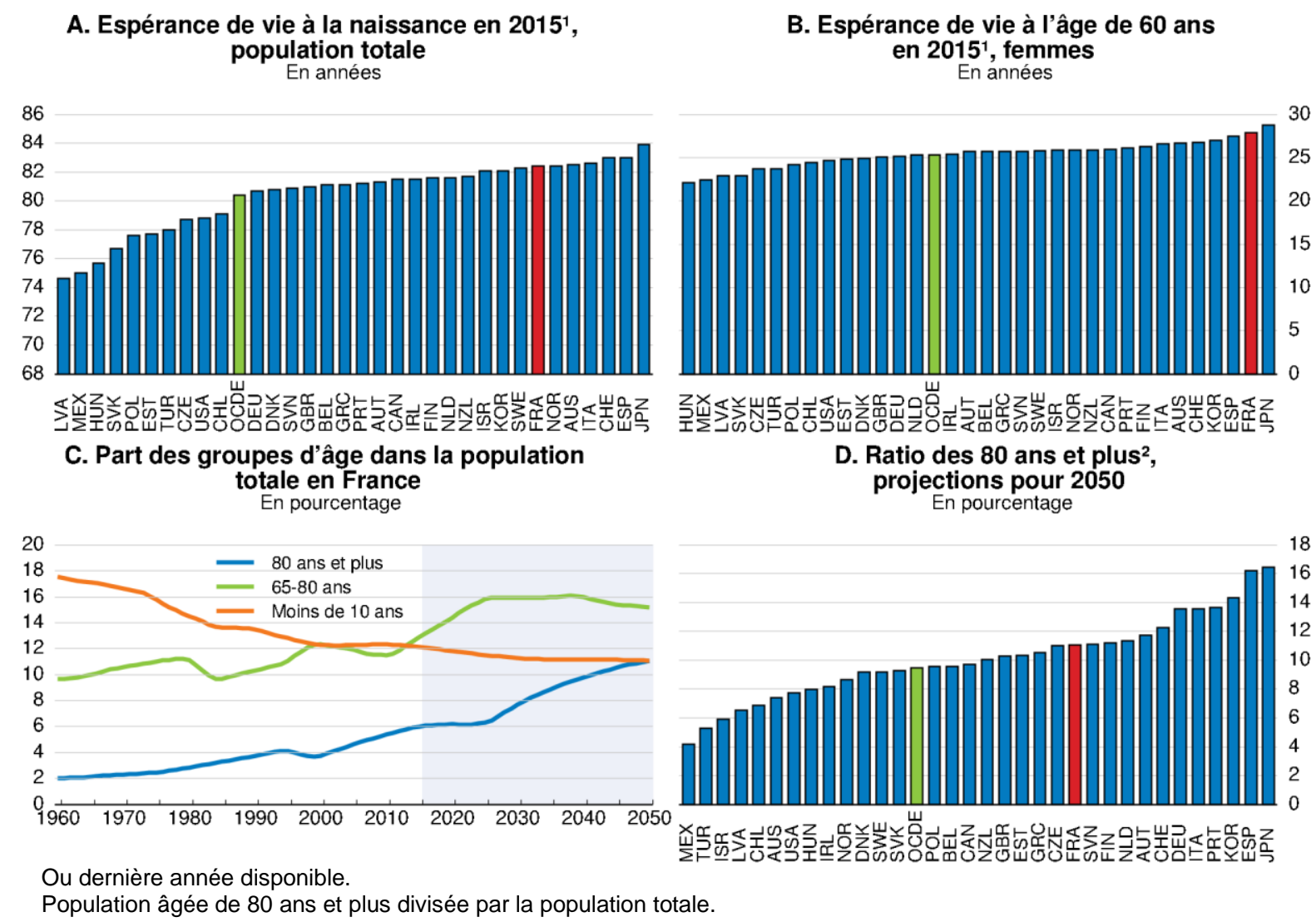

Source : OCDE (2017), Base de données des Statistiques de l'OCDE sur la santé ; OCDE (2017), Données historiques et projections de la population (1950-2050).

1. L'auteur est Antoine Goujard du Département des Affaires économiques de l'OCDE (antoine.goujard@oecd.org). L'auteur tient à remercier de nombreux collègues pour leurs commentaires, notamment Agnès Couffinhal et Valérie Paris (toutes les deux de la Direction de l'Emploi, du travail et des affaires sociales de l'OCDE) et Peter Jarrett, Nicola Brandt, Pierre Guérin, Falilou Fall, Robert Ford, Peter Hoeller, Jens Hoj et Alvaro Pereira (tous du Département des Affaires économiques de l'OCDE). Le rapport a aussi bénéficié des commentaires des experts du gouvernement français. L'auteur remercie également Patrizio Sicari pour son aide statistique et Claude-Annie Manga-Collard et Dacil Kurzweg pour l'édition (tous les trois sont au Département des Affaires économiques de l'OCDE). 
2060, bien que les estimations de ces dépenses supplémentaires varient fortement (encadré 1). Dans le même temps, l'accroissement de l'espérance de vie en bonne santé permettrait à ceux qui le souhaitent de travailler plus longtemps. Donner aux personnes âgées la possibilité et les incitations à travailler plus longtemps renforcerait leur bien-être matériel et la croissance à long terme (OCDE, 2014a).

\section{Encadré 1. Demande future de soins médicaux et de longue durée}

Comme le montrent les larges différences qui caractérisent les scénarios de dépenses présentés dans le tableau 1, il est très difficile de prévoir quels seront les besoins futurs en matière de santé et de soins de longue durée. Outre l'évolution de la démographie et de la situation économique, les dépenses de santé dépendront des éléments suivants :

- Le degré de vieillissement en bonne santé de la population, c'est-à-dire la mesure selon laquelle les gains d'espérance de vie se feront en bonne santé. Cette question dépendra étroitement de la nature du progrès médical, du développement des pratiques de prévention et des changements de modes de vie. Les projections de l'OCDE partent de l'hypothèse d'un vieillissement entièrement en bonne santé, alors que la Commission européenne (dans son scénario « de référence ») part de l'hypothèse d'un vieillissement en bonne santé seulement pour moitié.

- L'incidence du progrès technologique sur le coût des services de santé, tant du point de vue de la baisse des prix des services existants qu'en termes de création de services nouveaux et potentiellement chers. C'est ce dernier facteur qui prédomine depuis les dernières décennies, et qui contribue à l'augmentation des dépenses.

- L'élasticité de la demande de soins par rapport à la hausse des revenus (de combien les dépenses de santé augmenteront-elles si les revenus croissent de $1 \%$ ). Les études publiées divergent sur cette question et les projections de l'OCDE partent de l'hypothèse d'une élasticité plus faible (0.8) que la Commission européenne (1.05 en moyenne sur la période de référence).

- La tendance des rémunérations dans le secteur de la santé, comme dans d'autres secteurs de services, a augmenté aussi vite que dans le reste de l'économie malgré des gains de productivité inférieurs, avec à la clé une hausse relative du prix des services de santé (ce qu'on appelle « l'effet Baumol »).

- Des changements de société, par exemple liés à la volonté de s'occuper de ses parents dans le cadre familial.

- Des réformes, par exemple du suivi des affections de longue durée et de la rémunération des professionnels de santé, ce qui pourrait limiter la croissance des dépenses conformément au scénario de maîtrise des coûts de l'OCDE.

Tableau 1. Hausse prévue des dépenses publiques de santé en France, 2010-60

\begin{tabular}{llcc}
\hline & En pourcentage du PIB & $\begin{array}{c}\text { Soins } \\
\text { médicaux }\end{array}$ & $\begin{array}{c}\text { Soins de longue } \\
\text { durée }\end{array}$ \\
\hline OCDE, 2013 & Scénario de maîtrise des coûts & 2.2 & 0.6 \\
& Scénario de pression des coûts & 6.1 & 1.0 \\
\hline Commission européenne, 2015 & Scénario de référence & 0.9 & 0.8 \\
& Scénario des risques & 1.6 & 2.7 \\
\hline DG-Trésor - PROMEDE, 2013 & Scénario de référence & 2.0 (Dépenses publiques) \\
& Scénario de référence & 2.5 (Dépenses publiques et privées) \\
\hline
\end{tabular}

1. Pour la Commission européenne, hausse entre 2013 et 2060. Pour Promede, hausse entre 2011 et 2060.

Source : de la Maisonneuve, C. et J.O. Martins (2013), «Dépenses publiques de soins de santé et de soins de longue durée : Une nouvelle série de projections ", Documents d'orientation du Département des affaires économiques de l'OCDE, n 6 , Éditions OCDE, Paris ; Commission européenne (2015), Ageing Report 2015 ; Geay, C. et G. de Lagasnerie (2013), « Projection des dépenses de santé à l'horizon 2060, le modèle PROMEDE ", Documents de travail de la DG Trésor, $\mathrm{n}^{\circ} 8$.

Le système de santé dispense des soins de haute qualité, mais les gains d'efficience potentiels sont significatifs. Les décès qui pourraient être évités si le système de santé proposait un accès plus opportun et plus efficace à des services de haute qualité sont peu nombreux par rapport aux autres pays (graphique 2, partie A). La qualité des soins hospitaliers, mesurée par le taux de survie des patients hospitalisés pour un AVC ou une crise cardiaque, est supérieure à la moyenne de l'OCDE (partie B). Par conséquent, la qualité des soins perçue par le public est élevée (Commission européenne, 2014). Le 
faible taux d'hospitalisations évitables pour asthme et bronchopneumopathie chronique obstructive (BPCO) indique également qu'une prévention et des interventions de soins primaires adaptées sont en place (partie C). Toutefois, la France est en retard concernant certaines pratiques de prévention: l'hospitalisation évitable pour le diabète est élevée et les taux de vaccination pour les populations à risque sont en déclin (OECD/EU, 2016). Dans le même temps, le volume global d'antibiotiques prescrits est l'un des plus importants de l'OCDE (partie D), et le recours aux urgences hospitalières a augmenté de manière excessive : des soins primaires efficaces et accessibles auraient permis d'éviter près de $20 \%$ de ces visites (OCDE, 2016a).

\section{Graphique 2. Sélection d'indicateurs de la qualité des soins}

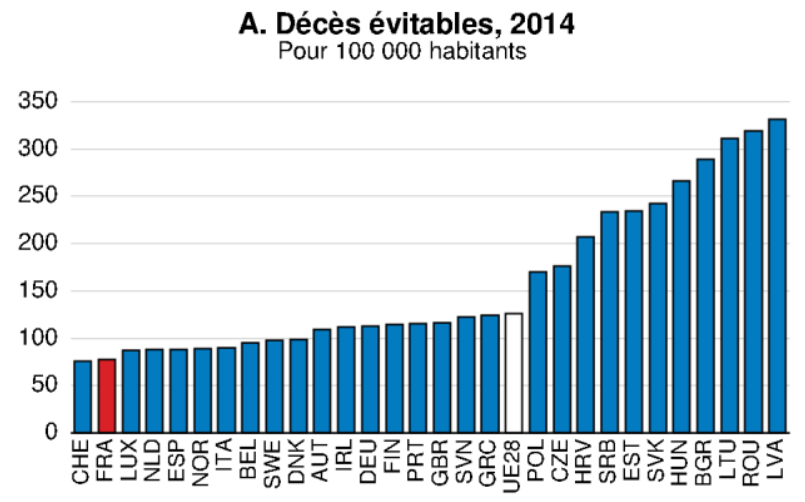

C. Admissions à l'hôpital pour asthme et BPCO, 2013'1 Taux standardisés par âge et sexe pour 100000 adultes

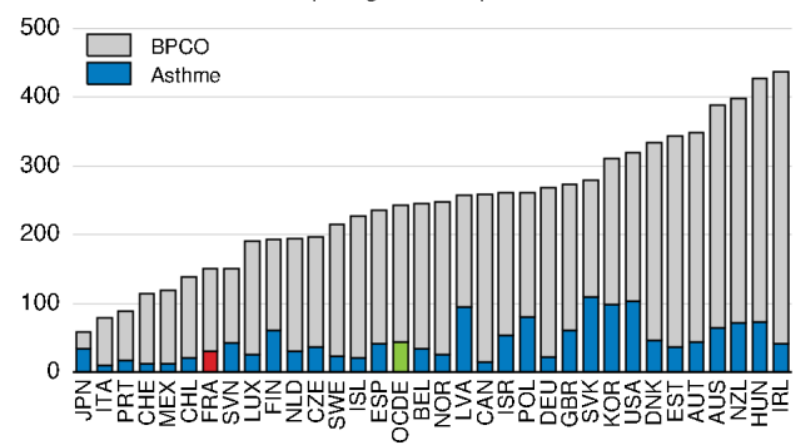

B. Mortalité liée aux infarctus aigus du myocarde, $2013^{1}$ Pour 1000 admissions $^{2}$

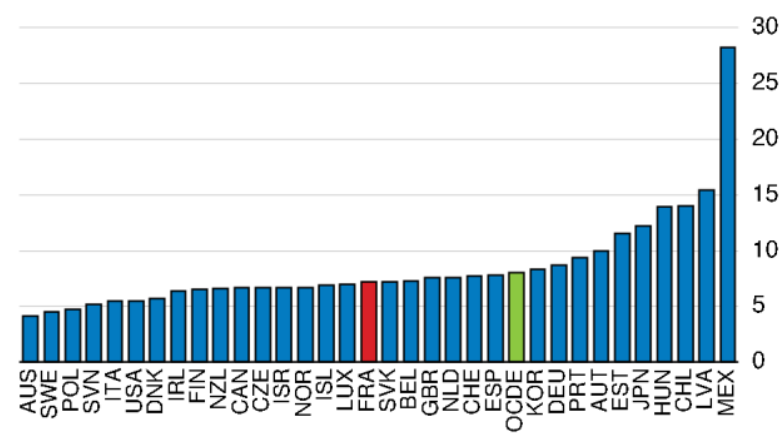

D. Volume total d'antibiotiques prescrits, $2013^{1}$ Doses quotidiennes définies pour 1000 habitants, par jour ${ }^{3}$

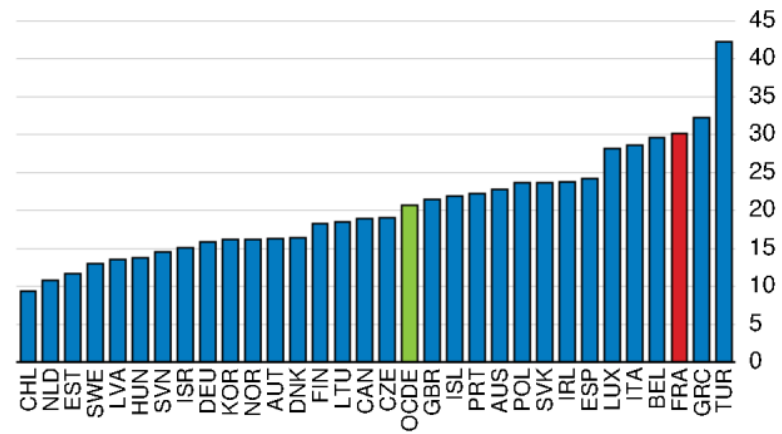

1. Ou dernière année disponible.

2. Mortalité dans les 30 jours suivant une hospitalisation pour infarctus aigu du myocarde. Taux standardisés par âge et sexe d'adultes âgés de 45 ans et plus, fondés sur les données relatives aux hospitalisations.

3. Dans les hôpitaux et les soins primaires. La dose quotidienne définie correspond à la dose moyenne supposée, par jour, d'un médicament utilisé dans son indication principale chez l'adulte (p. ex. 3 grammes pour l'aspirine par voie orale). Elle est constante d'un pays à l'autre.

Source: Eurostat (2017), Amenable and Preventable Deaths Statistics ; OCDE (2015), Panorama de la santé 2015 : Les indicateurs de l'OCDE, Éditions OCDE, Paris ; OCDE (2017), Base de données des Statistiques de l'OCDE sur la santé.

Les dépenses publiques et privées au titre des soins de santé sont relativement élevées. Ces dépenses ont augmenté régulièrement par rapport au PIB au cours des vingt dernières années, comme dans d'autres pays européens, pour s'établir à $11 \%$ du PIB en 2015 (graphique 3, parties A et B). Les dépenses au titre des soins curatifs et de réadaptation hospitaliers et des biens médicaux, notamment des produits pharmaceutiques, sont supérieures à la moyenne de l'OCDE (parties C et D). Les autorités ont mis en œuvre plusieurs mesures pour améliorer le contrôle des dépenses de santé et leur efficience. Toutefois, jusqu'en 2012, les limitations visaient principalement les dépenses publiques, ce qui avait probablement restreint la prise en charge des dépenses de santé des groupes les plus vulnérables qui n'ont pas accès aux assurances complémentaires privées, et avait réduit le contrôle des dépenses de santé globales à moyen terme (Askenazy et al., 2013 ; Bozio et Dormont, 2016). 
Graphique 3. Dépenses de santé

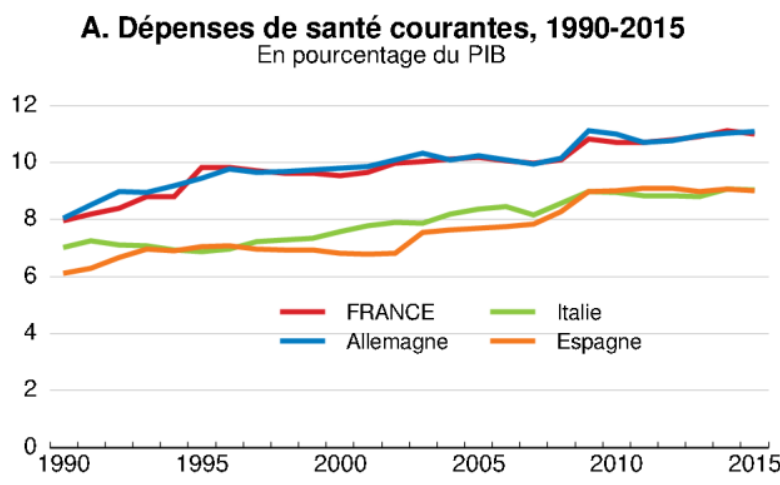

\section{Soins curatifs et de réadaptation hospitaliers, $2015^{2}$ En pourcentage du PIB}

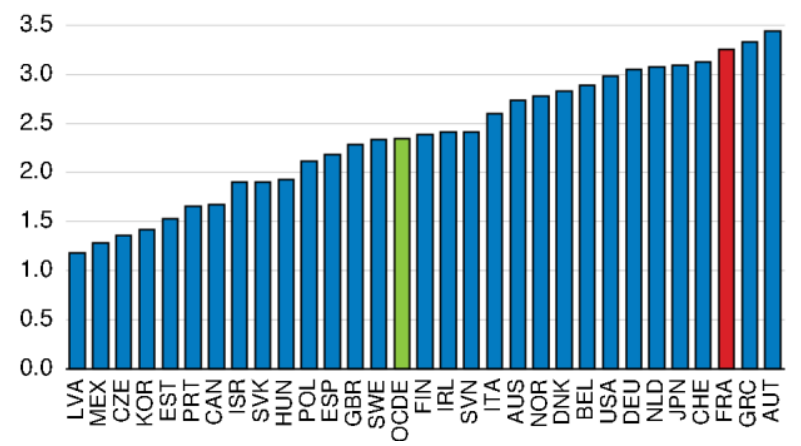

B. Dépenses de santé courantes, $2015^{1}$ En pourcentage du PIB

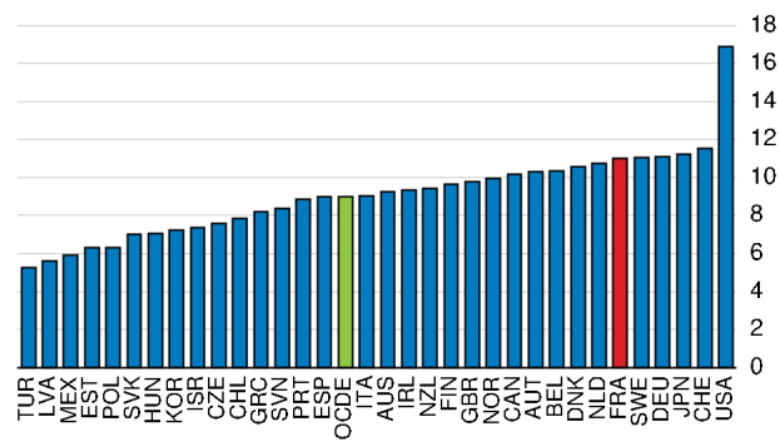

D. Biens médicaux, $2015^{2}$

En pourcentage du PIB

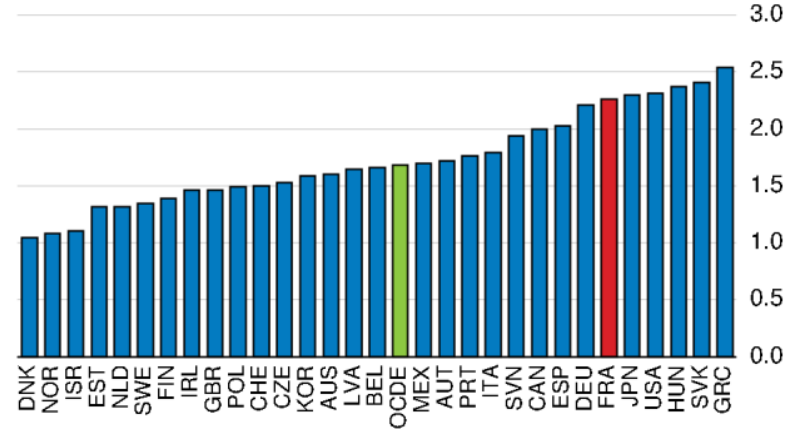

1. Les valeurs indiquées pour certains pays sont des estimations.

2. Ou dernière année disponible.

Source : OCDE (2017), Base de données des Statistiques de l'OCDE sur la santé.

Les disparités sociales et géographiques en termes de résultats de santé sont significatives, malgré de bons résultats agrégés. Les différences d'espérance de vie en fonction du niveau d'instruction ont diminué de façon constante au cours des 20 dernières années, et sont inférieures à la moyenne de l'OCDE (graphique 4, parties A et B). Toutefois, les différences d'espérance de vie en fonction de la catégorie socioéconomique n'ont pas convergé (partie C), et l'état de santé autoévalué des groupes peu qualifiés n'est pas bon (Pisarik et al., 2017). La différence entre le taux de mortalité des travailleurs manuels et celui des travailleurs non manuels semble importante par rapport aux autres pays (Kunst et al., 2001), et les disparités sur le plan de la santé sont significatives dès le plus jeune âge (Chardon et al., 2015). Les hommes français affichent un taux particulièrement élevé de décès prématurés par accident, ou dus à des habitudes néfastes pour la santé, comme le tabagisme et la consommation d'alcool, qui sont les causes les plus fréquentes de mortalité évitable. Il existe également d'importantes disparités géographiques : la différence d'espérance de vie entre les personnes vivant dans les départements affichant les taux de chômage les plus élevés et celles vivant dans les départements affichant les taux de chômage les plus bas a augmenté de six mois depuis le milieu des années 2000 (partie D).

Les disparités entre les groupes socioéconomiques et géographiques s'expliquent en partie par des différences de modes de vie et par la conception du système de santé. Les différences d'accès aux services de soins d'une région ou d'un quartier à l'autre, les restes à charge élevés pour certains ménages, l'hétérogénéité des pratiques médicales, notamment entre les hôpitaux, et le peu de place accordée à la prévention jouent également un rôle. Les délais d'attente pour obtenir un rendez-vous avec un spécialiste sont particulièrement longs dans certaines régions (Vergier, 2016). De plus, l'accès aux soins primaires est moins développé dans les quartiers les plus défavorisés (ONPV, 2016) et la qualité des soins 
hospitaliers demeure hétérogène (Gobillon et Milcent, 2013 et 2016). Le niveau relativement bas des dépenses de prévention peut entraîner une dégradation du bien-être des populations défavorisées et une hausse des coûts budgétaires à plus long terme.

\section{Graphique 4. Différences en matière de santé}

A. Différence d'espérance de vie à 35 ans entre les diplômés de l'enseignement secondaire et les non-diplômés En années

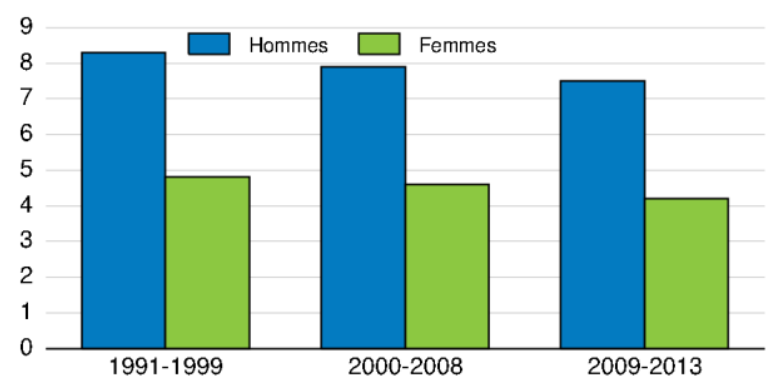

C. Différence d'espérance de vie à 35 ans entre travailleurs hautement qualifiés et travailleurs de la production ${ }^{2}$

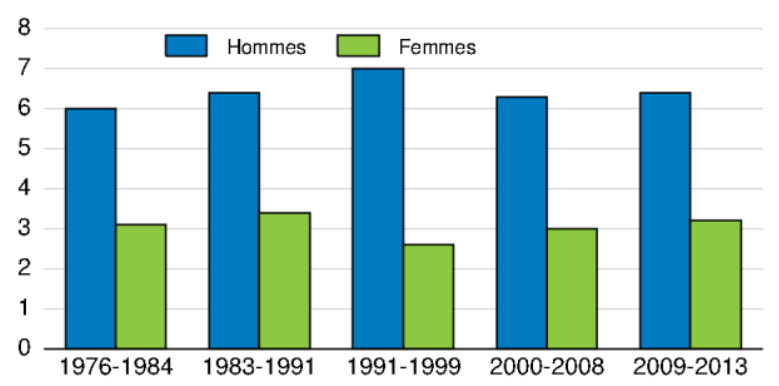

B. Différence d'espérance de vie à 25 ans entre les diplômés du secondaire et les personnes peu instruites, 2011' En années

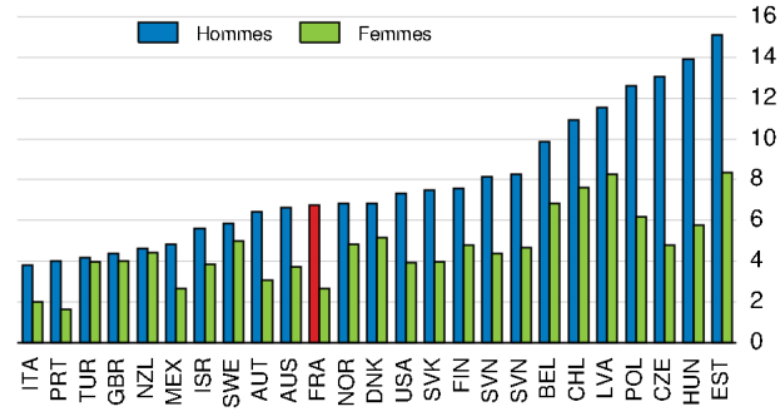

D. Différence d'espérance de vie à la naissance entre les populations vivant dans des régions de fort/faible chômage ${ }^{3}$ En années

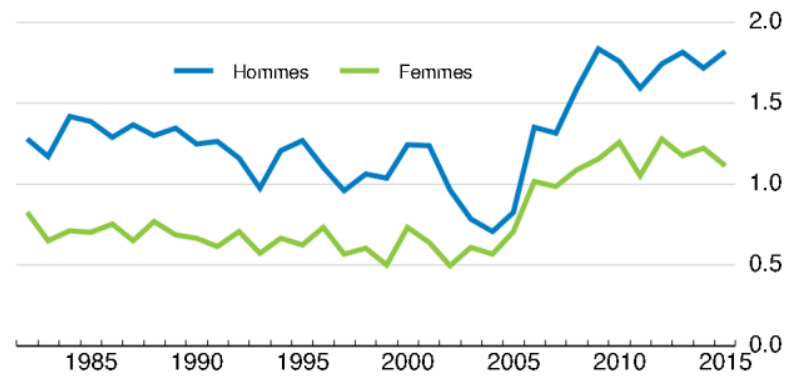

1. Les personnes peu instruites sont des personnes non diplômées ou titulaires d'un diplôme de l'enseignement primaire ou du premier cycle de l'enseignement secondaire.

2. Les travailleurs hautement qualifiés incluent les travailleurs non manuels et les travailleurs hautement qualifiés, comme les cadres, les chercheurs et les spécialistes des différents secteurs. Les travailleurs de la production regroupent les ouvriers qualifiés et non qualifiés.

3. Les régions correspondent aux départements de France métropolitaine et sont pondérées en fonction de leur population. Les régions où le chômage est élevé (faible) regroupent un tiers de la population.

Source: Blanpain, N. (2016), «Les hommes cadres vivent toujours 6 ans de plus que les hommes ouvriers », Insee Première, no 1584 ; Murtin, F., J. Mackenbach, D. Jasilionis et M. Mira d'Ercole (2017), « Inequalities in longevity by education in OECD countries: Insights from new OECD estimates », OECD Statistics Working Papers, n 2017/02, Éditions OCDE, Paris ; INSEE (2017), Banque de données macro- économiques (BDM) ; et calculs de l'OCDE.

Pour atteindre à long terme les objectifs multiples de soins de santé accessibles, de grande qualité et d'un coût abordable, il faudra compléter le contrôle budgétaire global par des incitations à rendre l'offre de soins de santé plus efficace, tout en faisant face à la hausse de la population âgée. Ce chapitre examine le cadre réglementaire du système de santé, avant de se concentrer sur des aspects spécifiques. Les principaux résultats sont les suivants :

- Le système de santé français obtient de bons résultats consolidés sur le plan de la santé, au prix d'importantes disparités sociales et d'un financement contraint. La France est également confrontée au vieillissement démographique et à la hausse des maladies chroniques. Le renforcement de la prévention et des soins primaires est donc prioritaire. Au-delà du système de santé, il est essentiel de réformer l'organisation des soins de longue durée (Bozio et al., 2016 ; OCDE/UE, 2016). 
- Le système de santé est complexe et des financements et services assurés par les secteurs public et privé y interviennent. Malgré un cadre budgétaire sain, le manque de coordination entre l'assurance maladie obligatoire et les organismes complémentaires limite le contrôle des dépenses et crée des disparités de couverture et d'accès aux soins.

- De nouveaux progrès sont nécessaires pour renforcer le contrôle des coûts par les prestataires de soins. La consommation de produits pharmaceutiques en général et d'antibiotiques en particulier est élevée, et certains mécanismes de rémunération et réglementations pourraient encourager davantage des soins appropriés et le regroupement des prestataires de soins. La poursuite des efforts d'amélioration de la coordination des prestataires de soins et de la prévention favoriserait des modes de vie plus sains et améliorerait les résultats à moyen terme sur le plan de la santé. Une gestion plus efficiente des ressources humaines et des achats d'équipements dans les hôpitaux engendrerait également des économies.

\section{Améliorer la gouvernance du système de santé}

Le système d'assurance français est fondé sur l'assurance universelle obligatoire et sur des systèmes d'assurance complémentaire facultatifs qui garantissent globalement un bon accès aux soins. Le reste à charge des ménages est relativement limité grâce à des dispositifs financés par les pouvoirs publics, qui couvrent les affections de longue durée et les ménages les plus pauvres. Toutefois, les dépenses privées ne sont pas plafonnées, et les différences entre les groupes de population demeurent importantes, notamment pour les chômeurs et les travailleurs âgés, tandis qu'un meilleur partage des remboursements de l'assurance maladie obligatoire et des organismes complémentaires pourrait inciter à plus d'efficacité.

\section{Améliorer le financement des dépenses de santé}

Le système d'assurance sociale et les organismes complémentaires d'assurance maladie jouent un rôle important dans le financement des dépenses de santé (graphique 5, parties A à C). L'assurance maladie obligatoire couvre l'ensemble de la population résidente grâce à un certain nombre de régimes. Le régime principal est la Caisse nationale d'assurance maladie des travailleurs salariés (CNAMTS). Elle couvre $85 \%$ de la population, principalement les salariés et les chômeurs, et est complétée par d'autres régimes spécifiques dont les principaux sont le régime social agricole et le régime social des indépendants. La CNAMTS joue un rôle central dans la rémunération et la gestion des professionnels de santé, puisqu'elle est en charge de tous les versements et de la collecte des prélèvements auprès des autres régimes obligatoires depuis 2016. En outre, $96 \%$ de la population dispose d'une complémentaire santé, par le biais des contrats collectifs d'entreprise, des assurances individuelles, ou dans environ $6 \%$ des cas par le biais d'un dispositif public (partie D).

La croissance des dépenses publiques a légèrement diminué depuis que le cadre budgétaire a été renforcé. En 1996, les autorités ont mis en place des objectifs annuels de dépenses dans le cadre de l'Objectif national des dépenses d'assurance maladie (ONDAM). L'assurance maladie obligatoire et les professionnels de santé s'appuient sur l'ONDAM pour fixer leurs objectifs en termes de dépenses et d'économies. Le suivi de ces objectifs s'est régulièrement amélioré, comme recommandé par l'OCDE (2011a), et la croissance effective des dépenses suit l'ONDAM de près depuis 2009 (encadré 2 ; graphique 6, partie A). Globalement, le cadre budgétaire semble sain (OCDE, 2015a), bien que la définition des dépenses tendancielles qui déterminent les économies à effectuer demeure insuffisamment documentée par sous-objectif selon la Cour des comptes (2015) et que l'ONDAM n'intègre pas les dépenses d'indemnités journalières de maternité et de paternité qui sont payées par l'assurance maladie et dont le montant élevé justifie un suivi rigoureux. 
Graphique 5. Le financement des dépenses de santé

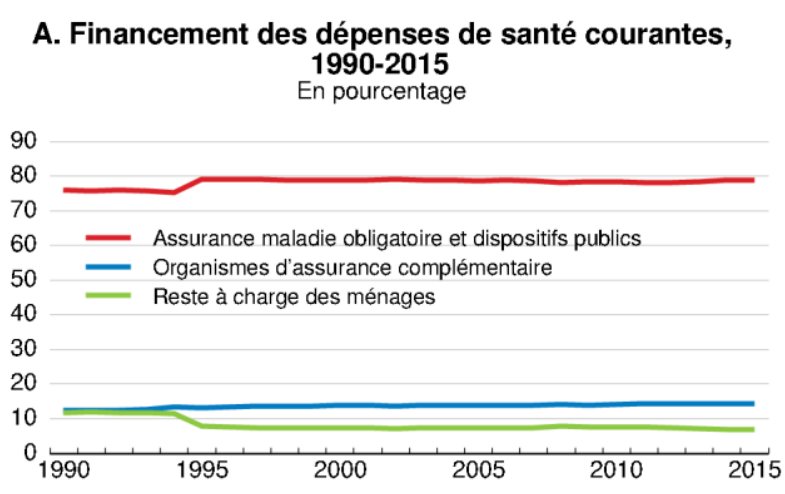
C. Dépenses de santé publique, 20151
En pourcentage du PIB

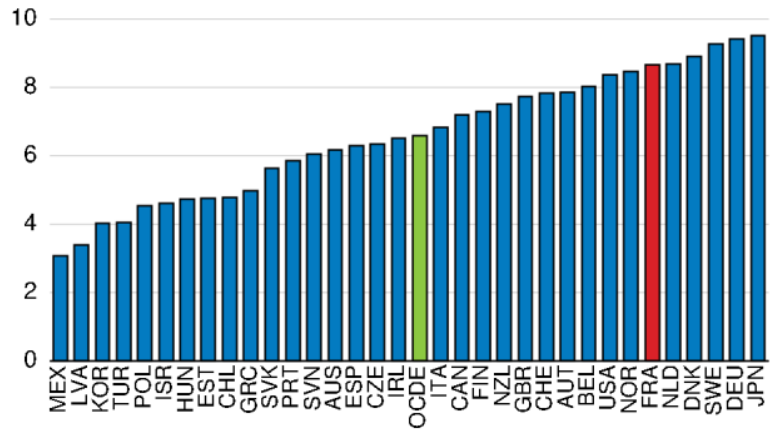

B. Financement des dépenses de santé courantes, 20151 En pourcentage

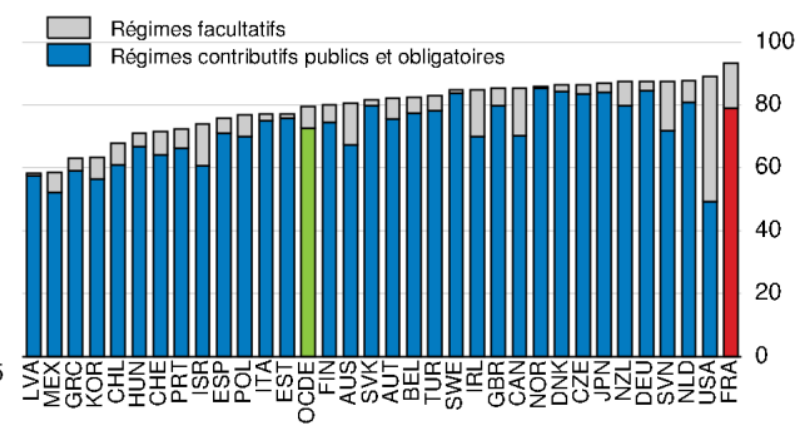

D. Couverture d'assurance maladie privée, 20141 En pourcentage de la population

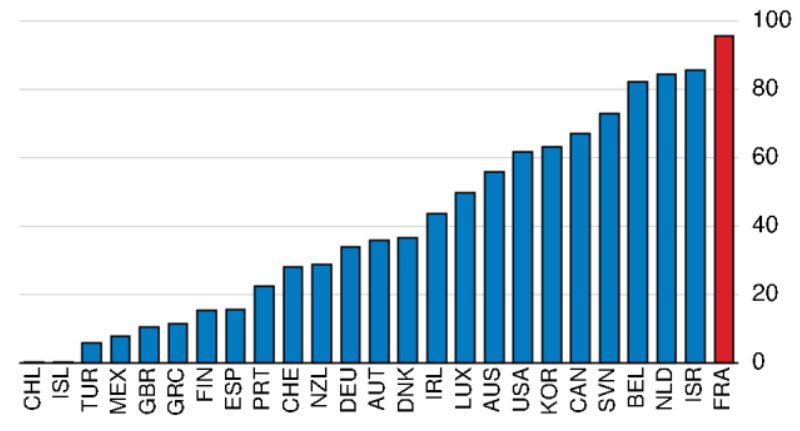

1. Ou dernière année disponible.

Source : OCDE (2017), Base de données des Statistiques de l'OCDE sur la santé.

\section{Encadré 2. Prévision des dépenses de santé publique et des objectifs annuels de dépenses (ONDAM)}

Le parlement vote les lois de financement de la sécurité sociale (LFSS) et l'Objectif national des dépenses d'assurance maladie (ONDAM) tous les ans. L'ONDAM porte sur les dépenses du système d'assurance maladie obligatoire et de l'État, soit environ $78 \%$ du total. Depuis 2014, et jusqu'en 2017, il était ventilé en six catégories : dépenses de soins de ville, dépenses hospitalières relatives à la tarification à l'activité (T2A), autres dépenses des établissements de santé, contribution aux dépenses des établissements et services pour personnes âgées, contribution aux dépenses des établissements et services pour personnes handicapées, et dépenses relatives aux autres modes de prise en charge. Depuis 2017, les deux catégories de dépenses hospitalières relatives à la tarification à l'activité (T2A) et autres dépenses des établissements de santé sont regroupées en une seule: dépenses des établissements de santé. Les objectifs définis par l'ONDAM demeurent indicatifs, sauf (en partie) pour les hôpitaux, puisque de nombreux acteurs prennent des décisions décentralisées en matière de dépenses.

Une procédure de suivi et d'adaptation à l'évolution des dépenses tout au long de l'année a été mise en place pour remédier au dépassement régulier de l'ONDAM au début des années 2000. Depuis 2004, un comité d'alerte avertit le parlement, le gouvernement et les caisses d'assurance maladie lorsque les dépenses d'assurance maladie risquent de dépasser l'ONDAM d'un montant égal à un pourcentage de l'objectif (seuil d'alerte). Ce pourcentage est fixé à $0.5 \%$ de l'objectif global depuis 2013. En cas de déclenchement de la procédure d'alerte, les caisses d'assurance maladie et le gouvernement proposent des mesures de redressement.

Une réserve de précaution sur les dotations peut être constituée au début de chaque année, et les professionnels de santé sont de plus en plus impliqués dans la gestion des dépenses de santé depuis 2010 . La réserve de précaution (au moins $0.3 \%$ de l'ONDAM convenu dans la loi de programmation des finances publiques 2014-2019) est versée aux établissements concernés (hôpitaux, FIR) en fonction de l'atteinte de l'objectif à la fin de l'année. Un comité de pilotage se réunit chaque mois pour encourager la coopération entre les ministères de la Santé et du Budget et la Caisse d'assurance maladie, et éviter les avertissements du comité d'alerte.

Source : OCDE (2015a), Fiscal Sustainability of Health Systems, Bridging Health and Finance Perspectives, Éditions OCDE, Paris ; PLFSS (2015), Projet de loi de financement de la sécurité sociale pour 2016, ministère des Finances et des Comptes publics 
Le gouvernement espère maintenir le taux de croissance de l'ONDAM à un niveau historiquement bas sur la période 2017-20. Cette restriction des dépenses permettrait de combler le déficit de la CNAMTS en 2019 (graphique 6), sous réserve de la concrétisation de la hausse significative attendue de l'emploi et des salaires, qui se traduirait par une hausse des recettes (Le Gouvernement, 2016 ; HCFI-PS, 2017a). La loi de modernisation de notre système de santé, en date de 2016, prévoit plusieurs moyens de réduire les dépenses : développement des soins ambulatoires, amélioration de l'efficience des dépenses hospitalières, poursuite des efforts pour abaisser le coût des médicaments et promouvoir les médicaments génériques, renforcement des paiements fondés sur des incitations, et renforcement de l'usage efficace et approprié des soins primaires et de l'hospitalisation. Cette approche est bienvenue, dans la mesure où elle pourrait pérenniser les restrictions des dépenses.

\section{Graphique 6. Dépenses de santé publique}

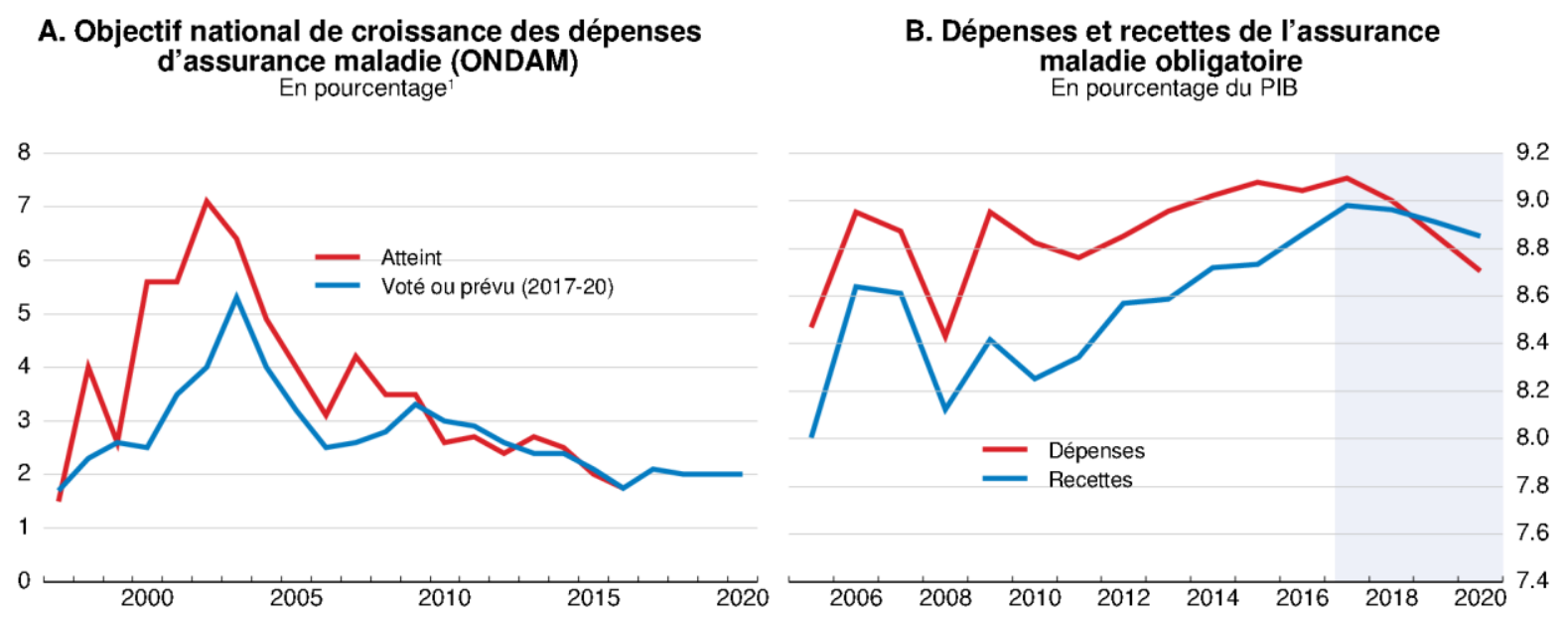

1. Valeurs nominales.

Source: Commission des comptes de la sécurité sociale (2016), Les comptes de la sécurité sociale : Résultats 2015 et prévisions 2016, juin ; Loi de financement de la sécurité sociale (LFSS) 2017.

Les dépenses de santé des ménages atteints d'affections de longue durée sont associées à des risques considérables sur le plan du budget et de l'équité à moyen terme. Le régime d'assurance maladie obligatoire prend entièrement en charge toutes les dépenses engendrées par une maladie chronique figurant sur une liste d'affections de longue durée (ALD). La prévalence des ALD est particulièrement élevée chez les plus de 70 ans (graphique 7). Les dépenses des patients avec une ALD pour leur maladie chronique et leurs autres soins de santé représentaient $4.4 \%$ du PIB en 2014 (PLFSS, 2016). Pourtant, l'assurance maladie couvre à $100 \%$ uniquement la partie de ces dépenses qui est directement liée à la maladie chronique des patients ALD. Cela représente seulement autour de $14 \%$ du total de leurs dépenses de santé (Caby et Eidelman, 2015). Pour cette raison, le reste à charge est généralement important pour les patients atteints d'une affection de longue durée. Les bénéficiaires en ALD représentaient un tiers des ménages affichant le reste à charge le plus élevé (tranche supérieure de $5 \%$ ) en 2010 (HCAAM, 2013).

Le financement de l'assurance maladie obligatoire repose à la fois sur des cotisations et des impôts. Les cotisations assises sur les salaires (prélevées principalement auprès des employeurs) ont progressivement diminué (graphique 8, partie A). Une taxe plus générale, la CSG (Contribution sociale généralisée), a gagné en importance et est prélevée sur les sources de revenu, comme les salaires, les revenus 
des actifs financiers et des investissements, les retraites, les prestations de chômage, les prestations d'invalidité et les gains au jeu. Une vingtaine de taxes affectées à des dépenses spécifiques sur des bases relativement étroites, comme les taxes sur la consommation de produits nocifs, tels que le tabac et l'alcool, complètent le financement des soins de santé (OCDE, 2015a). Cette diversité des prélèvements limite la transparence pour les entreprises et les ménages (HCFI-PS, 2015), et accroît la charge administrative pesant sur les entreprises, qui ont affaire à différentes institutions de collecte des taxes et cotisations sociales, même si des procédures électroniques simplifiées sont en place, comme la déclaration sociale nominative (DSN) généralisée en janvier 2017 pour accroître la transparence et limiter la charge administrative (voir plus bas). Des mesures de simplification permettant notamment de diminuer le coin fiscalo-social pourraient intervenir (comme la suppression des cotisations salariales maladie). Au-delà, une poursuite de la simplification est envisageable : elle pourrait tendre à unifier les diverses taxes comportementales d'une part, et à accroître, d'autre part le financement basé sur l'impôt à très large assiette qu'est la CSG au détriment des cotisations assises sur le seul facteur travail. Cela contribuerait notamment à réduire les disparités entre les salariés, les chômeurs et les travailleurs indépendants en matière d'assurance maladie (HCFI-PS, 2017b).

Graphique 7. Dispositif spécial pour les affections de longue durée (ALD), 2014

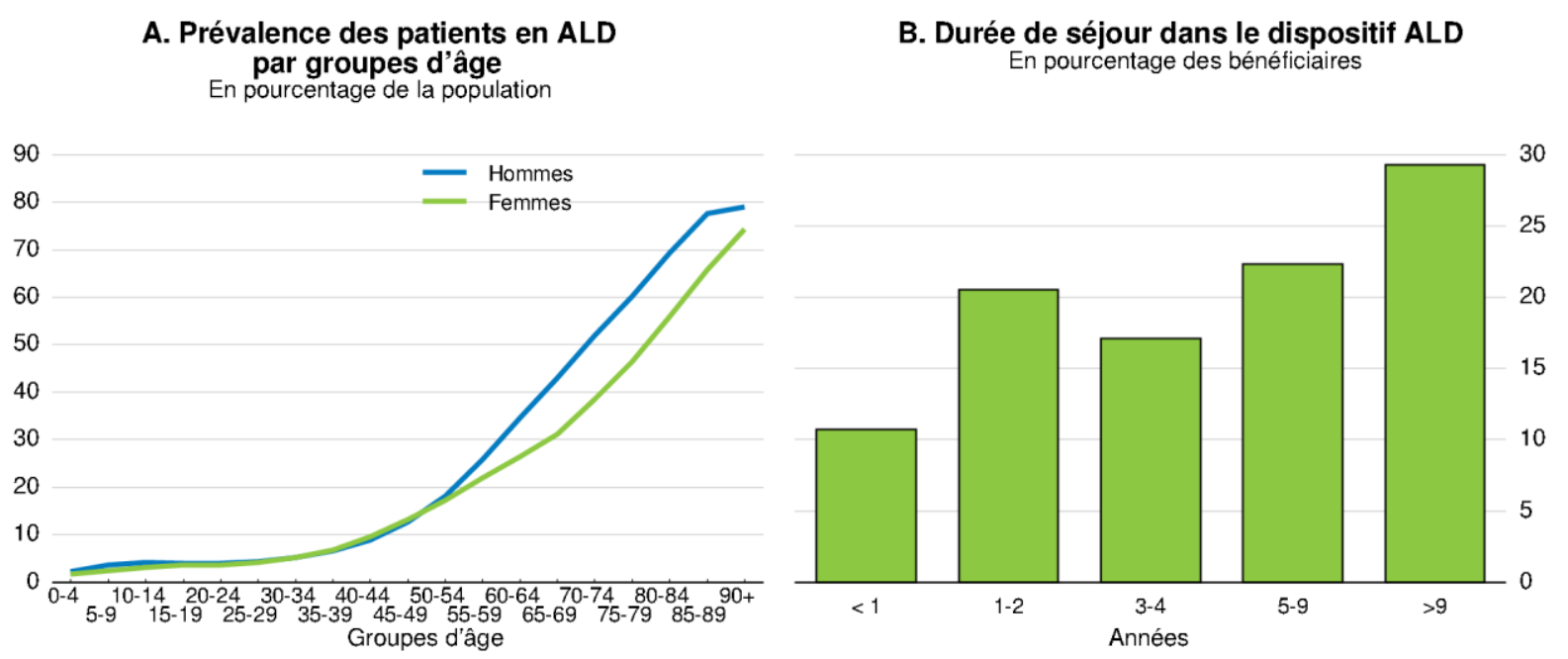

Source: Païta, M., S. Rivière, S. Tala et A. Fagot-Campagna (2016), "Les bénéficiaires du dispositif des affections de longue durée en 2014 et les évolutions depuis 2005 ", Points de repère, no 46 ; Sécurité sociale (2016), Répartition des personnes en ALD au 31 décembre 2014 selon l'ancienneté de leur ALD.

Les organismes complémentaires d'assurance maladie financent le reste à charge après remboursement par les régimes d'assurance obligatoire. Pour certains types de soins, étant donné les taux de remboursement relativement faibles de l'assurance obligatoire, les assurances complémentaires sont essentielles pour l'accès aux soins. Ils couvrent tout ou partie du reste à charge après intervention de l'assurance obligatoire, ce qui n'est pas le cas dans plusieurs autres pays européens (Askenazy et al., 2013 ; Paris et al., 2016). Certains organismes complémentaires sont des entreprises privées à but lucratif, alors que d'autres sont des organisations à but non lucratif (mutuelles et caisses d'assurance). Dans le cadre actuel, les organismes complémentaires couvrent généralement le reste à charge pour de nombreux services et ils ont peu d'impact sur les l'organisation des soins. Ils ont établi des réseaux encore peu développés de professionnels de santé, qui couvrent uniquement des dépenses spécifiques peu remboursées par le régime obligatoire, comme les soins optiques, dentaires et les audioprothèses (HCAAM, 2013). En effet, ils n’ont pas le droit de passer des accords avec les médecins généralistes (Pierron, 2016). 


\section{Graphique 8. Le système de santé est redistributif}

Par déciles de revenu disponible de I (décile inférieur) à $X$ (décile supérieur)

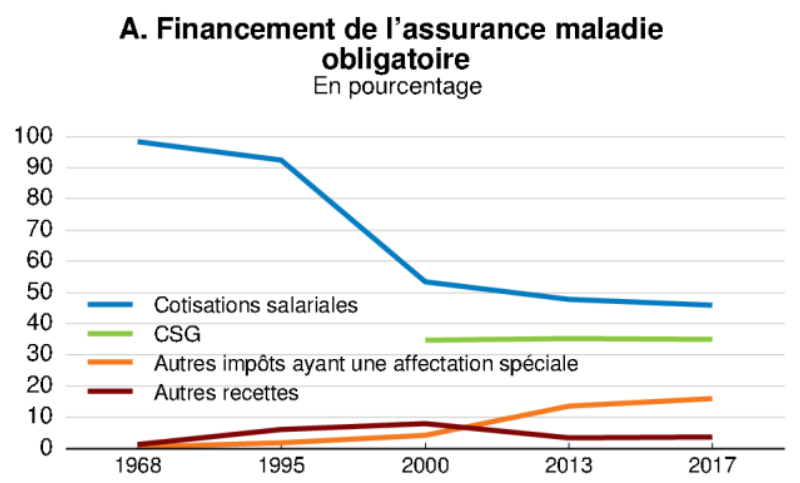

C. Remboursements par décile de revenu, 2012 En pourcentage du revenu disponible

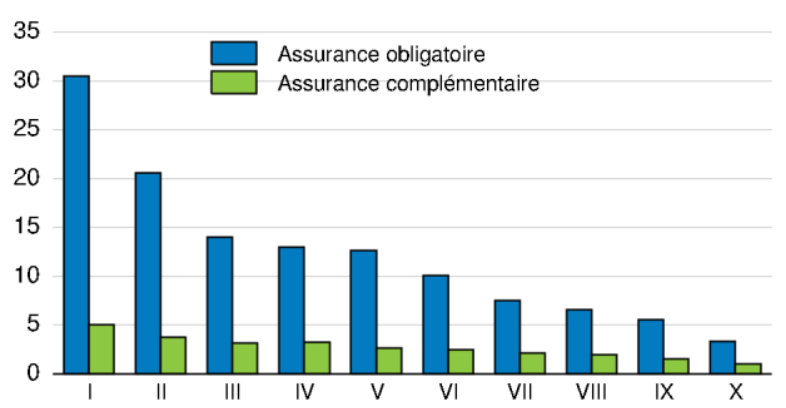

B. Financement de l'assurance maladie par décile de revenu, 2012 En pourcentage du revenu disponible

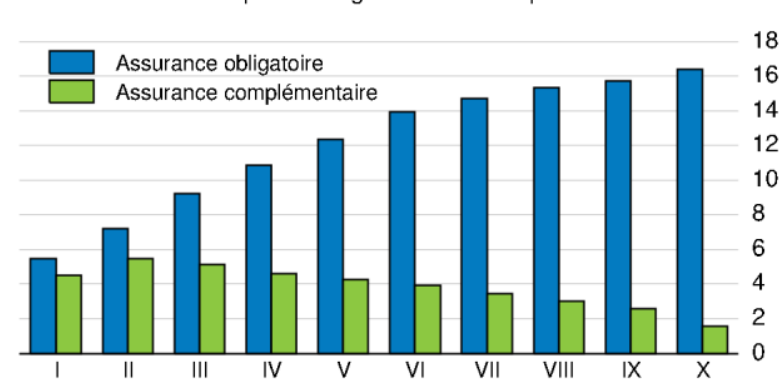

D. Reste à charge par décile de revenu, 2012 En pourcentage du revenu disponible

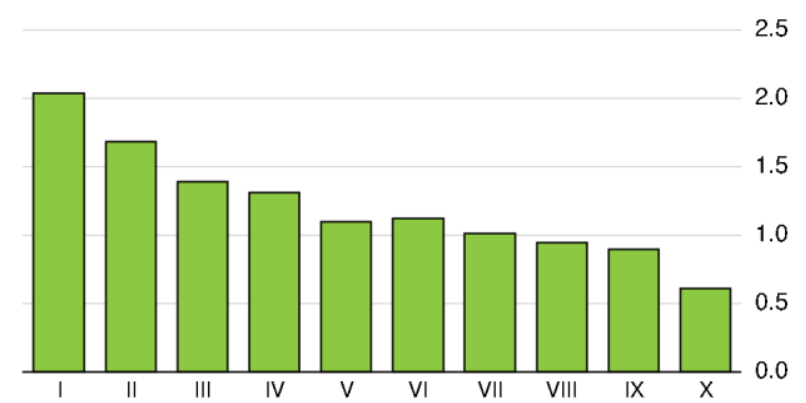

Source : Drees (2016), La complémentaire santé : acteurs, bénéficiaires, garanties - Édition 2016 et Les dépenses de santé en 2015 - Résultats des comptes de la santé ; Commission des comptes de la Sécurité sociale (2014, 2016 et 2017), Les comptes de la Sécurité sociale - Résultats 2014 ; Les comptes de la Sécurité sociale - Résultats 2015 et État des lieux actualisé du financement de la protection sociale - janvier 2017.

Les assurances complémentaires et les autorités ont pour objectif de rendre plus efficient le parcours de soins, grâce à des incitations financières et au rôle renforcé du médecin traitant. Comme recommandé par l'OCDE (OECD, 2000 ; Imai et al., 2000), les autorités ont mis en place différentes formes de tickets modérateurs, pratiques de non- remboursement, franchises et tarifs forfaitaires afin de limiter la croissance des dépenses de santé publique. Par exemple, les patients doivent payer 1 euro par consultation médicale (non ré-assurable par les contrats de complémentaire solidaires et responsables, témoignant d'une politique de coordination entre assurances obligatoire et complémentaires), et les taux de remboursement de certains services médicaux dépendent du parcours de soins emprunté. En particulier, le régime d'assurance maladie obligatoire rembourse une part moindre des soins dentaires et optiques et des consultations des spécialistes pratiquant des dépassements d'honoraires. Un forfait hospitalier est également appliqué.

La couverture des assurances obligatoires et complémentaires varie considérablement d'un prestataire et d'un traitement à l'autre. En particulier, concernant les soins primaires, le financement est principalement assuré par l'assurance maladie obligatoire (64\% en 2015) (tableau 2). Les organismes complémentaires prennent en charge, sauf rares exceptions, l'ensemble des tickets modérateurs depuis la réforme des contrats responsables intervenue en 2014. Si la multiplication des niveaux de tickets modérateurs peut nuire à la lisibilité du système pour les ménages, la prise en charge systématique des tickets modérateurs par les contrats responsables permet de limiter le renoncement aux soins. En outre, la pratique obligatoire du tiers payant pour les populations fragiles (CMU-C, ACS et AT-MP, mais aussi ALD et personnes prises en charge au titre de la maternité depuis 2017), facilite également l'accès aux soins. 
Tableau 2. Structure du financement des différents produits et services médicaux, 2006-15

\begin{tabular}{|c|c|c|c|c|c|c|c|c|c|}
\hline & \multirow{2}{*}{$\begin{array}{r}\begin{array}{c}\text { Part des } \\
\text { dépenses }\end{array} \\
2015\end{array}$} & \multicolumn{2}{|c|}{$\begin{array}{c}\text { Assurance } \\
\text { maladie } \\
\text { obligatoire }\end{array}$} & \multicolumn{2}{|c|}{ CMU-C et État ${ }^{1}$} & \multicolumn{2}{|c|}{$\begin{array}{c}\text { Organismes } \\
\text { complémentaires }\end{array}$} & \multicolumn{2}{|c|}{ Ménages } \\
\hline & & 2006 & 2015 & 2006 & 2015 & 2006 & 2015 & 2006 & 2015 \\
\hline Soins hospitaliers & 55.2 & 92.0 & 91.3 & 1.1 & 1.2 & 4.4 & 5.2 & 2.4 & 2.3 \\
\hline Hôpitaux publics & 42.9 & 92.5 & 92.0 & 1.4 & 1.4 & 4.1 & 4.8 & 2.0 & 1.8 \\
\hline Soins primaires & 44.8 & 63.9 & 64.2 & 1.6 & 1.6 & 20.0 & 20.4 & 14.5 & 13.8 \\
\hline Services médicaux & 21.9 & 63.4 & 64.7 & 1.9 & 2.0 & 21.0 & 21.7 & 13.8 & 11.7 \\
\hline Soins dentaires & 2.4 & 35.7 & 33.4 & 3.0 & 3.6 & 34.7 & 40.3 & 26.6 & 22.7 \\
\hline Médicaments & 16.0 & 68.5 & 68.8 & 1.5 & 1.5 & 16.5 & 12.8 & 13.6 & 17 \\
\hline Autres biens médicaux 2 & 4.1 & 41.7 & 43.1 & 0.8 & 0.8 & 32.6 & 39.0 & 24.9 & 17.3 \\
\hline Transports de malades & 2.8 & 92.7 & 93.1 & 0.8 & 0.9 & 3.9 & 3.8 & 2.6 & 2.2 \\
\hline Dépenses de santé & 100.0 & 76.8 & 76.8 & 1.4 & 1.4 & 12.8 & 13.3 & 9.0 & 8.4 \\
\hline
\end{tabular}

1. La CMU-C et l'aide médicale de l'État sont des régimes publics visant à compléter l'assurance maladie obligatoire et les régimes complémentaires pour les ménages les plus pauvres et les immigrés.

2. Optique, prothèses, orthèses, véhicules pour handicapés physiques, et autres petits matériels.

Source: Drees (2016), Les dépenses de santé en 2015 - Résultats des comptes de la santé, Drees.

Le système de santé engendre une redistribution considérable entre les groupes de revenu, principalement par le biais de son financement. Il réduit les inégalités en termes de revenu disponible des ménages, comme le montre le coefficient de Gini, de 0.3 à 0.25 d'après les simulations du ministère des Finances (Duval et Lardellier, 2012). Les primes d'assurance complémentaire ne sont que faiblement décroissantes du revenu (graphique 8, partie B). Dans le même temps, les remboursements des assurances obligatoires et complémentaires sont également progressifs, même si la part du reste à charge dans le revenu disponible des ménages à bas revenu est plus élevée que celle des autres ménages (parties $\mathbf{C}$ et $\mathbf{D}$ ).

\section{Réduire les différences de couverture et renforcer les incitations visant les professionnels de santé}

Trois dispositifs principaux visent à aider les ménages pauvres et les immigrés à accéder aux soins de santé. L'Aide médicale d'Etat (AME), financée directement par les pouvoirs publics, prend en charge un large panier de soins pour les résidents en situation irrégulière. Par ailleurs, deux autres dispositifs facilitent l'accès aux assurances complémentaires. Les ménages les plus défavorisés $(7.6 \%$ de la population en 2014) ont droit à la couverture maladie universelle complémentaire (CMU-C). Ce dispositif offre une couverture complémentaire gratuite aux bas revenus, et son plafond de revenu a été considérablement relevé en 2013. Les autres groupes socio-économiques défavorisés peuvent bénéficier de l'aide à l'acquisition d'une complémentaire santé (ACS) pour financer leurs primes d'assurance complémentaire. Toutefois, le dispositif ACS est très peu utilisé. En 2014, le taux de recours est d'environ 30 à $43 \%$ (Bruant-Bisson et Daudé, 2016) et, même parmi ses bénéficiaires, $20 \%$ ne s'en servaient pas pour souscrire un contrat d'assurance complémentaire (graphique 9, partie A).

L'accès aux organismes complémentaires est inégal. Les chômeurs, les primo-arrivants sur le marché du travail et les travailleurs âgés ont plus difficilement accès à l'assurance complémentaire (graphique 9, partie B). Ils ont également davantage recours aux contrats individuels qu'aux contrats collectifs d'entreprise, plus généreux (parties $\mathbf{C}$ et $\mathbf{D}$ ). Dans le même temps, des avantages fiscaux importants ont contribué au développement des contrats collectifs d'entreprise offrant une meilleure couverture avec des remboursements plafonnés à un niveau élevé récemment (Bruant-Bisson et Daudé, 2016). Et les contrats collectifs d'entreprise ont été rendus obligatoires pour les salariés du secteur privé en 2016, ce qui a permis des extensions de la couverture des soins. La séparation des contrats individuels et des contrats collectifs limite cependant la mutualisation des risques entre les salariés et les ménages plus à risque. En effet, la généralisation des contrats collectifs pour les salariés contribue à une concentration des ménages à risque plus élevé dans les contrats individuels. Il est possible que la portabilité des droits limite 
ce phénomène à long terme, même si elle engendre actuellement une augmentation du coût du contrat importante pour l'assuré lors du passage à la retraite.

\section{Graphique 9. Accès à la complémentaire santé}
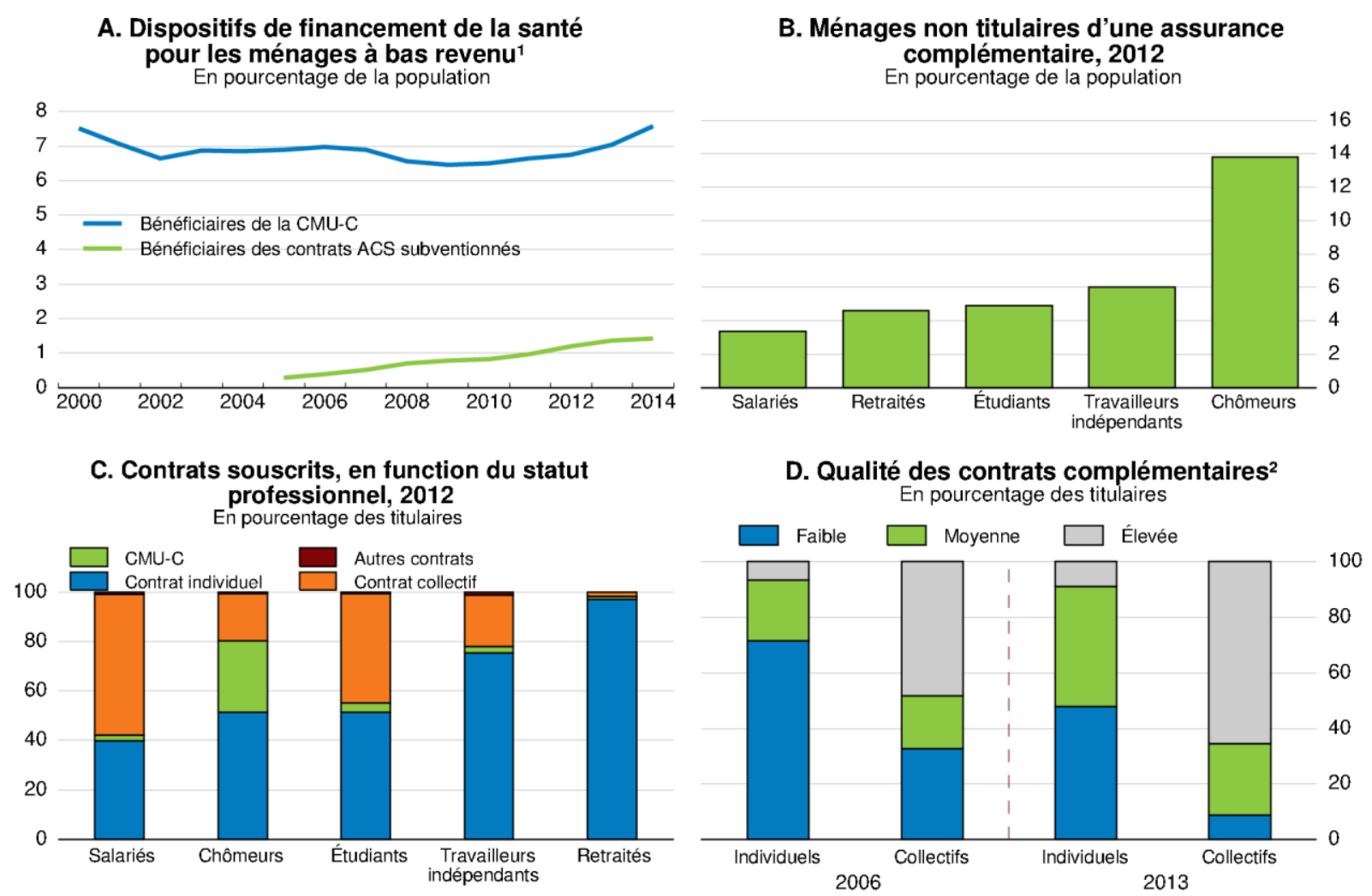

1. Une extrapolation rétrospective en 2000-2005 est appliquée aux séries des bénéficiaires de la CMU-C 2001 sur la base des données Eco- santé.

2. Le ministère de la Santé évalue la qualité des contrats selon le remboursement d'un panier de biens donnés ; un regroupement en cinq classes homogènes a été adopté, de $\mathrm{A}$ (remboursements les plus élevés) à $\mathrm{E}$. Les contrats offrant la moins bonne couverture correspondent aux classes $\mathrm{D}$ et $\mathrm{E}$, ceux offrant une couverture moyenne à la classe $\mathrm{C}$, et ceux offrant la meilleure couverture aux classes A et B.

Source: Drees (2016), La complémentaire santé : acteurs, bénéficiaires, garanties - Édition 2016, Drees ; bases de données Eco Santé et calculs de l'OCDE.

Les autorités ont pris certaines mesures pour améliorer l'accès aux assurances complémentaires pour les retraités et les ménages pauvres. Elles ont récemment élargi l'accès des ménages pauvres aux dispositifs d'aide pour favoriser l'accès aux soins (CMU-C et ACS). La réforme de l'ACS a conduit à la sélection d'un nombre limité de contrats d'assurance complémentaires qui proposent des remboursements standardisés. Elle a également supprimé les paiements directs pour les ménages remplissant les conditions requises. Depuis 2008, les droits à la portabilité de la complémentaire santé de l'ancien employeur ont été progressivement accrus pour les nouveaux retraités et des personnes qui ont perdu leur travail. Des incitations fiscales encouragent l'élaboration de contrats d'assurance complémentaire collectifs solidaires et responsables qui ne discriminent pas en fonction de l'âge, et garantissent des remboursements minimum et maximum. Dans le même temps, la discrimination selon les risques de santé n'est autorisée ni pour les contrats collectifs ni individuels.

Ces mesures ont conduit à une hausse de la part de la population titulaire d'une assurance complémentaire, mais elles sont ambiguës pour ce qui est des populations les plus vulnérables. Par exemple, les allègements d'impôt sur le revenu des sociétés et des personnes physiques pour les contrats 
collectifs offrant une bonne couverture ont encouragé les dépassements d'honoraires, en dépit des plafonds pour les remboursements. Les dépassements d'honoraires augmentent les restes à charge des personnes moins bien couvertes. La suppression progressive des allègements d'impôt sur les contrats collectifs permettrait d'économiser 2.35 milliards d'euros $(0.1 \%$ du PIB en 2014) (Bruant-Bisson et Daudé, 2016), mais elle pourrait augmenter le coût du travail. De nouvelles réformes permettraient également d'améliorer l'accès à l'assurance complémentaire pour les ménages à bas revenu et leur santé à moyen terme (CNAMTS, 2016a), notamment en promouvant l'utilisation des données administratives existantes sur les revenus et les autres dépenses sociales pour déterminer et contrôler l'accès à l'ACS. En effet, les expérimentations menées par le passé ont montré que les ménages remplissant les critères d'accès, principalement les chômeurs de longue durée et les retraités, pouvaient être difficiles à joindre par le biais des campagnes d'information (Guthmuller et al., 2012). La soutenabilité du dispositif ACS pourrait être améliorée en partie en en revoyant les critères d'accès (Cour des comptes, 2015).

Les organismes complémentaires limitent la participation au coût pour les dépenses sur lesquelles les patients exercent un certain contrôle, comme certains médicaments et les consultations de médecins pratiquant des honoraires élevés. Ceci peut stimuler la croissance des dépenses et les prix, et exclure les groupes les plus vulnérables. En effet, les remboursements conjoints de l'assurance maladie obligatoire et des organismes complémentaires pour un même panier de soins limitent les incitations à améliorer l'efficience des dépenses de santé, les ménages ayant des difficultés à faire la distinction entre les différents taux de remboursement, certains contrats complémentaires prenant aussi en charge l'intégralité des tickets modérateurs (tableau 2). L'amélioration de la couverture des organismes complémentaires semble avoir entraîné un accroissement de la demande de spécialistes avec des honoraires élevés (Dormont et Péron, 2016). De même, la récente diminution des taux de remboursement des médicaments et d'indemnisation des arrêts maladie n'a pas réduit leur utilisation, ce qui suggère que ces dépenses de santé des ménages ne sont pas sensibles au prix en France (Davezies et Toulemon, 2015 ; Pollack, 2015).

Pour augmenter l'efficacité de la participation des patients aux coûts, la Haute Autorité de santé (HAS), en charge de l'évaluation des biens et procédures médicaux, est chargée de définir une liste de médicaments, dispositifs et procédures médicaux qui seraient remboursés par l'assurance maladie obligatoire et leur taux de remboursements. Ceci devrait renforcer les liens entre les taux de remboursements et l'évaluation de l'efficacité clinique et économique des nouveaux médicaments (voir ci-dessous). Dans un premier temps, l'assurance maladie obligatoire pourrait arrêter de rembourser les médicaments autorisés dont l'efficacité est incertaine. Une réforme plus ambitieuse du système d'assurance maladie pourrait séparer les produits et les services médicaux couverts par l'assurance obligatoire d'un côté et par les assurances complémentaire de l'autre (Askenazy et al., 2013). Ceci faciliterait la négociation avec les professionnels de santé. Les contrats d'assurance complémentaire se spécialiseraient ainsi dans des biens et services médicaux «optionnels », comme les soins optiques, certains soins dentaires et les audioprothèses. Un désengagement de l'Assurance maladie obligatoire de secteurs où son financement est minoritaire, tels le dentaire, l'optique et l'audioprothèse, poserait toutefois des questions délicates : risques d'anti-sélection et d'augmentation des prix et perte de contrôle sur les dépenses concernées.

Les autorités devraient davantage durcir les normes relatives aux «contrats responsables » des assurances complémentaires et en améliorer la lisibilité pour renforcer la concurrence. En effet, les assurances complémentaires utilisent potentiellement des moyens indirects (différentes gammes de contrats) pour parvenir à une segmentation de leur clientèle par groupes de risques en dépit de l'interdiction de sélection selon les risques de santé. Cet encadrement renforcé réduirait les incitations à la consommation des soins des individus en meilleure santé ainsi que les dépassements d'honoraires, notamment via un plafond plus bas pour les remboursements. Les seniors et les ménages plus pauvres en bénéficieraient. En effet, les compagnies d'assurance ont favorisé les populations en meilleure santé en proposant des contrats spécifiques et en appliquant différentes structures de primes: par 
conséquent, les coûts de santé des ménages augmentent considérablement avec l'âge (graphique 10 ; Drees, 2016a), même dans le cas des mutuelles à but non lucratif (Leduc et Montaut, 2016). Un système avec des termes contractuels plus règlementés limiterait les possibilités pour les assurances complémentaires de sélectionner leurs clients en fonction de leurs risques de santé. Ils seraient ainsi en concurrence sur le montant des primes pour les différents groupes de populations et de risques. Dans un tel système les assureurs devraient conclure des contrats avec des professionnels de santé et organiser des parcours de soins plus efficaces. Ceci demanderait une vraie mutualisation des risques, par exemple via un système de péréquation des risques entre assureurs pour assurer la couverture de tous et limiter la hausse potentielle des primes d'assurance tout en prenant en compte le libre choix des prestations.

\section{Graphique 10. Accès aux régimes complémentaires d'assurance maladie par groupes d'âge}

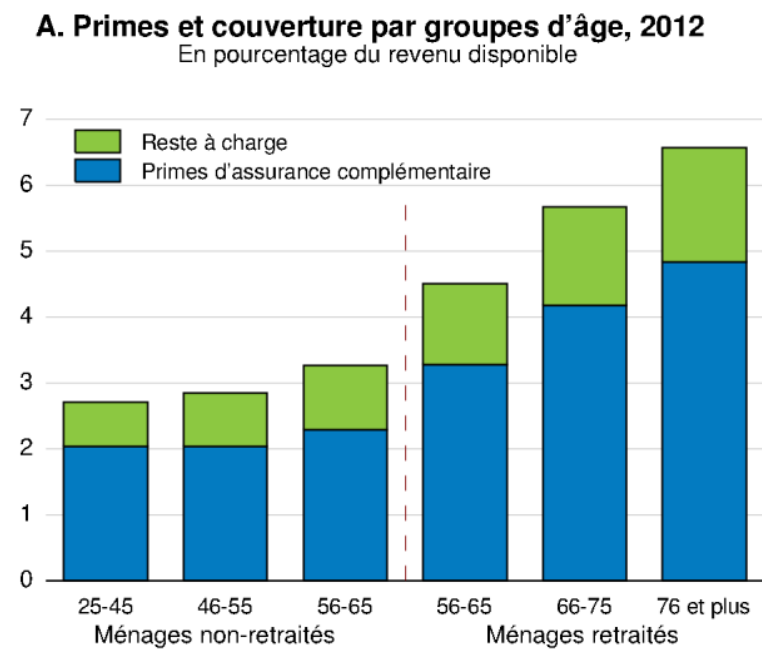

B. Qualité des contrats complémentaires, 2012
En pourcentage

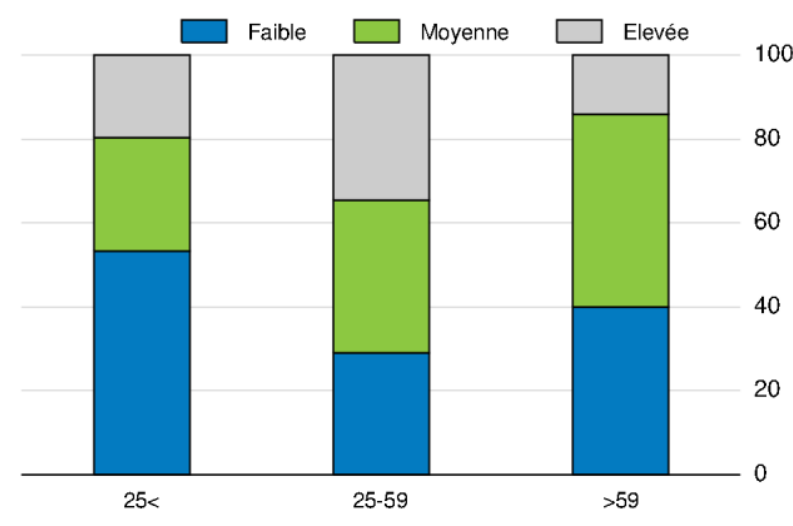

1. Le ministère de la Santé évalue la qualité des contrats selon le remboursement d'un panier de biens donnés ; un regroupement en cinq classes homogènes a été adopté, de $A$ (remboursements les plus élevés) à $E$. Les contrats offrant la moins bonne couverture correspondent aux classes $\mathrm{D}$ et $\mathrm{E}$, ceux offrant une couverture moyenne à la classe $\mathrm{C}$, et ceux offrant la meilleure couverture aux classes $\mathrm{A}$ et $\mathrm{B}$.

Source : Drees (2016), La complémentaire santé : acteurs, bénéficiaires, garanties - édition 2016.

Dans le contexte d'une réforme ambitieuse de l'assurance de santé complémentaire la limitation du reste à charge des ménages après remboursement par l'assurance maladie obligatoire pourrait améliorer à la fois l'équité et l'efficience. Ce reste à charge pourrait être plafonné en fonction du revenu des ménages, comme dans d'autres pays de l'OCDE (Paris et al., 2010) et remplacerait le dispositif ALD. Contrairement à l'ALD, le plafond ne dépendrait pas des affections ni de la situation professionnelle du patient, ce qui lui offrirait une meilleure protection contre les risques de santé et limiterait les effets potentiellement négatifs des bas revenus sur l'accès aux soins. Toutefois, un plafond bas menacerait l'attractivité des polices d'assurance complémentaire et nécessiterait une vaste réforme du système.

\section{Diminuer les coûts administratifs}

Les remboursements conjoints des dépenses de santé compliquent la gestion des remboursements et l'organisation du financement du système de santé. Les coûts de gestion de soins sont plus élevés en France que dans la plupart des autres pays de l'OCDE (graphique 11). Les coûts administratifs des assurances obligatoires et complémentaires sont à peu près équivalents, mais les assurances complémentaires financent une part bien moindre des dépenses de santé totales. Leurs coûts comparativement élevés s'expliquent en partie par les frais de marketing et de recherche, les frais de gestion - $5.4 \%$ des prestations versées - étant proches des $3.5 \%$ de la CNAMTS (Auvigné et al., 2013). 
Graphique 11. Les dépenses administratives au titre de la santé sont élevées

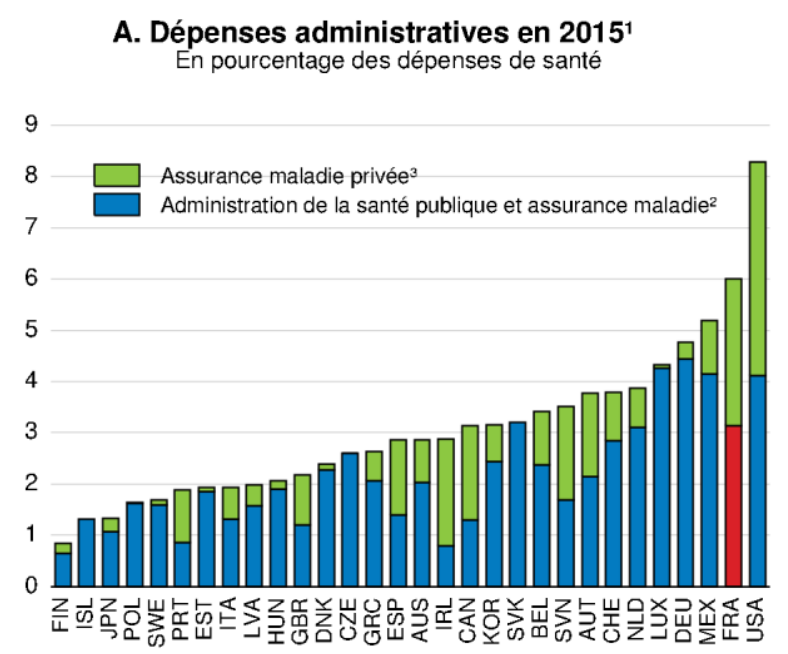

\section{B. Dépenses administratives, 2000-15 En pourcentage des dépenses de santé}

1. Ou année la plus proche.

2. Y compris l'assurance maladie obligatoire gérée par les assureurs privés.

3. Fait principalement référence aux régimes d'assurance maladie volontaire privée.

Source : OCDE (2017), Base de données des Statistiques de l'OCDE sur la santé.

L'harmonisation de la collecte des prélèvements et l'amélioration de la gestion des organismes locaux d'assurance maladie obligatoire permettraient de faire quelques économies. En ce sens, la protection universelle maladie a été renforcée en 2016 pour simplifier et harmoniser la gestion administrative. L'organisation de l'assurance maladie obligatoire demeure fragmentée sur le plan local, bien que la rémunération des prestataires par les différents régimes obligatoires ait été harmonisée en 2016 (Auvigné et al., 2013 ; Cour des comptes, 2016a). Certains régimes obligatoires disposent de leur propre réseau de collecte et peuvent associer la gestion de l'assurance maladie et d'autres dépenses de sécurité sociale (p.ex. risques professionnels, retraites et certaines prestations familiales). Les caisses locales de la CNAMTS remboursent les services médicaux, étudient les demandes des patients et s'occupent d'autres tâches administratives. Toutefois, elles sont parfois en charge de populations et de territoires très vastes, et affichent des performances variables (Auvigné et al., 2013). La réduction du nombre de régimes spéciaux, outre la convergence des différentes structures administratives vers de meilleures pratiques, permettrait de réduire la complexité et d'améliorer l'efficience et la coordination, notamment en ce qui concerne la collecte des prélèvements (Cour des comptes, 2015).

\section{Améliorer l'organisation des prestations de soins}

Une nouvelle approche centrée sur la réponse aux besoins des malades pour l'organisation de l'offre de soins permettrait de mieux gérer les pressions liées au vieillissement démographique et à la hausse de la prévalence des maladies chroniques, tout en réduisant les inégalités d'accès aux soins. Les données internationales récentes de l'OCDE montrent que certains aspects du côté de l'offre, comme la rémunération des professionnels de santé et les réglementations, et du côté de la demande, comme le contrôle de l'accès aux soins et la participation au coût, peuvent jouer un rôle essentiel dans la régulation des dépenses de santé (de la Maisonneuve et al., 2016). Des médecins généralistes et spécialistes bien formés, répartis de façon homogène à travers le pays, sont également essentiels pour garantir une offre adéquate de soins de ville, et la coordination avec les hôpitaux et les autres prestataires de soins primaires (OCDE, 2016b). 


\section{Améliorer la coordination de l'offre de soins}

La France dispose d'un système de prestations assuré par le public et privé dans lequel les patients sont libres de choisir le prestataire. La gestion de l'offre de soins est partiellement décentralisée, et les organismes complémentaires, les hôpitaux et les professionnels des soins primaires sont incités à coordonner les parcours de soins des patients au niveau local, via plusieurs mesures telles que le développement des maisons et centres de santé, des plateformes territoriales d'appui, des projets régionaux de soins et le dispositif Paerpa qui vise à améliorer la prise en charge coordonnée des personnes âgées en perte d'autonomie. Les résultats sont mitigés sur le plan de l'organisation malgré le déploiement de programmes d'actions nationaux. Les dépenses hospitalières et de soins primaires augmentent rapidement (tableau 3), alors que les dépenses au titre de la prévention institutionnelle accusent un retard, notamment par rapport aux dépenses de santé totales (graphique 12, partie A). En outre, les hospitalisations pour asthme et les bronchopneumopathies chroniques obstructives sont relativement peu fréquentes, mais celles liées à un diabète qui pourrait être parfaitement traité en ville sont supérieures à la moyenne de l'OCDE (partie B), et le recours aux urgences a augmenté durant les vingt dernières années, reflétant pour partie l'organisation insuffisante de la permanence des soins ambulatoires et une utilisation dévoyée du système de soins hospitaliers par les malades (partie C). En 2013, la part des bénéficiaires de soins de longue durée âgés de 65 ans et plus recevant des soins à domicile était inférieure à la moyenne de l'OCDE (OCDE, 2015b).

Les autorités envisagent plusieurs réformes concernant l'offre de soins de santé. Elles avaient prévu de doubler la part des soins de longue durée à domicile d'ici à 2018. Toutefois, ils restent moins développés que dans la plupart des autres pays comparables, et les mesures prises par le passé n'ont pas permis d'accroître le niveau des soins à domicile ni de parvenir à une offre géographiquement équilibrée (Cour des comptes, 2016b). De même, certaines formes de chirurgie ambulatoire demeurant relativement moins développées que dans d'autres pays de l'OCDE (graphique 12, partie D), le gouvernement envisage d'accroître la part de la chirurgie ambulatoire pour progresser de $50 \%$ du total des interventions en 2015 à plus de deux tiers en 2020, ce qui nécessitera un réseau de médecins généralistes et de personnel infirmier bien formés, une coordination formelle entre les prestataires de soins primaires et les hôpitaux (mise en place en 2016 par la loi de modernisation de notre système de santé), et l'adaptation des rémunérations et des réglementations existantes (Bert et al., 2014 ; ATIH, 2016).

La coordination de l'offre de soins au niveau régional a progressé. En 2010 les Agences régionales de santé (ARS) ont rassemblé des entités autrefois distinctes pour les hôpitaux et la médecine en ville. Les ARS sont chargées de s'assurer que les acteurs régionaux répondent aux besoins de la population en améliorant la coordination entre les secteurs hospitaliers et des soins primaires et les services sociaux et de santé, tout en respectant l'ONDAM. Les ARS ont une certaine influence sur l'organisation des services hospitaliers, notamment sur le nombre de patients qu'ils peuvent accueillir. Le gouvernement envisage de soutenir des parcours de soins plus intégrés impliquant les hôpitaux et les acteurs de soins primaires. La loi sur la santé de 2016 a donné un cadre pour le développement des équipes de soins primaires et envisage de renforcer les communautés professionnelles territoriales de santé en charge de la coordination de ces soins sous la surveillance des ARS, via la constitution de plateformes territoriales d'appui. Toutefois, l'influence 
Tableau 3. Structure des dépenses de santé, 1995-2015

\begin{tabular}{|c|c|c|c|c|c|c|c|c|}
\hline & 1995 & 2000 & 2007 & 2010 & 2012 & 2013 & 2014 & 2015 \\
\hline \multicolumn{9}{|l|}{ A. Dépenses en part du PIB } \\
\hline 1-Soins hospitaliers & 3.9 & 3.6 & 3.7 & 4.0 & 4.0 & 4.1 & 4.1 & 4.2 \\
\hline Hôpitaux publics & 2.9 & 2.8 & 2.9 & 3.1 & 3.1 & 3.1 & 3.2 & 3.2 \\
\hline Hôpitaux privés & 1.0 & 0.8 & 0.9 & 0.9 & 0.9 & 0.9 & 0.9 & 1.0 \\
\hline 2-Soins primaires & 3.9 & 4.1 & 4.4 & 4.7 & 4.7 & 4.7 & 4.7 & 4.8 \\
\hline Services primaires & 2.1 & 2.0 & 2.1 & 2.2 & 2.2 & 2.3 & 2.3 & 2.3 \\
\hline Transports de malades & 0.1 & 0.1 & 0.2 & 0.2 & 0.2 & 0.2 & 0.2 & 0.2 \\
\hline Produits pharmaceutiques & 1.4 & 1.6 & 1.7 & 1.7 & 1.7 & 1.6 & 1.6 & 1.6 \\
\hline Autres biens médicaux ${ }^{2}$ & 0.3 & 0.4 & 0.5 & 0.6 & 0.6 & 0.6 & 0.6 & 0.7 \\
\hline 3-Soins de longue durée & . & 0.5 & 0.7 & 0.9 & 0.9 & 0.9 & 0.9 & 0.9 \\
\hline 4-Indemnités de maladie & . & 0.6 & 0.6 & 0.6 & 0.6 & 0.6 & 0.6 & 0.6 \\
\hline 5-Autres dépenses pour les patients & . & 0.0 & 0.0 & 0.0 & 0.0 & 0.0 & 0.0 & 0.0 \\
\hline 6-Prévention & . & 0.3 & 0.3 & 0.3 & 0.3 & 0.3 & 0.3 & 0.3 \\
\hline 7-Dépenses pour le système de santé & . & 0.5 & 0.5 & 0.6 & 0.6 & 0.6 & 0.6 & 0.6 \\
\hline Recherche & . & 0.4 & 0.4 & 0.4 & 0.4 & 0.4 & 0.4 & 0.3 \\
\hline 8-Coûts de gestion du système de santé & 0.7 & 0.7 & 0.6 & 0.7 & 0.7 & 0.7 & 0.7 & 0.7 \\
\hline Consommation de services et de biens $(1+2)$ & 7.9 & 7.7 & 8.2 & 8.7 & 8.7 & 8.7 & 8.8 & 8.9 \\
\hline Dépenses de santé actuelles $(1+\ldots+8)^{1}$ & . & 10.2 & 10.9 & 11.8 & 11.8 & 11.9 & 12.1 & 12.0 \\
\hline $\begin{array}{l}\text { Dépenses de santé actuelles - définition de } \\
\text { |'OCDE }\end{array}$ & 9.8 & 9.5 & 10.0 & 10.7 & 10.8 & 10.9 & 11.1 & 11.0 \\
\hline \multicolumn{9}{|c|}{ B. Dépenses, taux de croissance réels annualisés } \\
\hline 1-Soins hospitaliers & . & 0.5 & 1.7 & 2.9 & 2.6 & 1.9 & 2.3 & 2.1 \\
\hline Hôpitaux publics & . & 1.1 & 1.4 & 2.9 & 2.7 & 2.0 & 2.1 & 2.0 \\
\hline Hôpitaux privés & . & -1.3 & 2.4 & 3.0 & 2.2 & 1.7 & 2.9 & 2.2 \\
\hline 2-Soins primaires & . & & & 2.8 & 2.9 & 2.4 & 3.8 & 2.8 \\
\hline Services primaires & . & 2.5 & 3.6 & 3.4 & 3.2 & 3.3 & 1.5 & 2.7 \\
\hline Transports de malades & . & 3.1 & 5.9 & 4.0 & 2.9 & 2.7 & 6.6 & 3.6 \\
\hline Produits pharmaceutiques & . & 5.0 & 6.9 & 3.9 & 4.5 & 3.6 & 3.9 & 4.1 \\
\hline Autres biens médicaux & . & 8.1 & 6.7 & 2.9 & 2.6 & 1.9 & 2.3 & 2.1 \\
\hline 3-Soins de longue durée 3 & . & & 5.7 & 6.2 & 2.6 & 3.0 & 1.9 & 1.4 \\
\hline 4-Indemnités de maladie 3 & . & & 1.9 & 3.4 & -0.5 & -0.4 & 3.6 & 1.6 \\
\hline 5-Autres dépenses pour les patients ${ }^{3}$ & . & & . & 5.9 & 10.9 & 6.0 & 0.0 & -2.5 \\
\hline 6-Prévention ${ }^{3}$ & . & & 2.1 & 0.8 & -1.0 & 1.7 & -0.8 & -1.6 \\
\hline 7-Dépenses pour le système de santé ${ }^{3}$ & . & & 2.6 & 1.6 & 1.1 & 2.0 & -1.3 & 0.1 \\
\hline Recherche $^{3}$ & 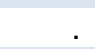 & & & -0.5 & -0.5 & 0.1 & -0.3 & -0.6 \\
\hline 8-Coûts de gestion du système de santé ${ }^{3}$ & 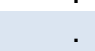 & 2.4 & 1.6 & 2.4 & 1.6 & 2.9 & 0.3 & -1.9 \\
\hline Consommation de services et de biens $(1+2)$ & $\cdot$ & 2.3 & 3.6 & 2.8 & 2.8 & 2.2 & 3.1 & 2.5 \\
\hline Dépenses de santé actuelles $(1+\ldots+8)^{1,3}$ & 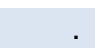 & & 2.7 & 2.1 & 1.4 & 1.3 & 1.9 & 0.9 \\
\hline $\begin{array}{l}\text { Dépenses de santé actuelles - définition de } \\
\text { l'OCDE }^{3}\end{array}$ & . & 2.3 & 2.5 & 2.1 & 1.6 & 1.6 & 2.0 & 0.6 \\
\hline
\end{tabular}

1. Dépenses de santé actuelles, définition nationale.

2. Optique, prothèses, orthèses, véhicules pour handicapés physiques, et autres petits matériels.

3. Déflaté par le déflateur du PIB.

Source : Calculs réalisés par l'OCDE à partir des Comptes nationaux de la santé (bases 2000, 2005 et 2010) ; Le Garrec, M.-A., M. Koubi et A. Fenina (2013), “ 60 années de dépenses de santé », Études et résultats, $\mathrm{n}^{\circ} 831$, Drees ; Base de données de l'OCDE sur les comptes nationaux; OCDE (2017), Base de données des Statistiques de l'OCDE sur la santé.

des ARS sur les soins primaires restera limitée, puisque la plupart des rémunérations et réglementations relèvent de décisions nationales. Il serait utile de leur donner plus d'autonomie pour passer des accords avec les professionnels de santé en vue de la coordination des parcours de soins. Elles devraient pouvoir piloter l'organisation des soins primaires dans leur région et pourraient être financées en fonction des besoins des populations régionales, qui feraient l'objet d'estimations régulières. Elles auraient alors plus de latitude pour coordonner les soins primaires, les hôpitaux et les services médicosociaux, notamment si elles 
étaient autorisées à reporter pour leur propre usage les économies réalisées chaque année. Par exemple, les ARS pourraient financer les services médicosociaux par la vente groupée de biens et services pour des réseaux des professionnels de santé, et développer la rémunération des structures communes pour encourager la coopération, bien que nombre de ces mesures soient actuellement définies à l'échelle nationale.

\section{Graphique 12. Sélection d'indicateurs de prévention et de coordination entre les prestataires de soins}

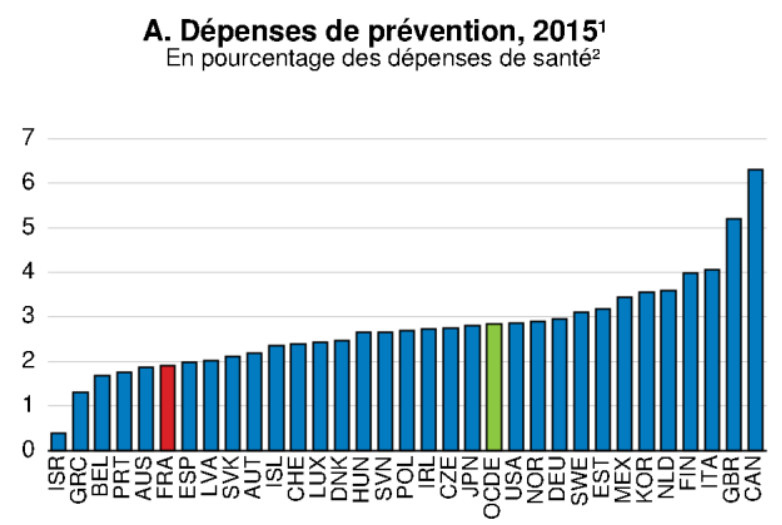

C. Utilisation des urgences hospitalières Indice, 1996=100

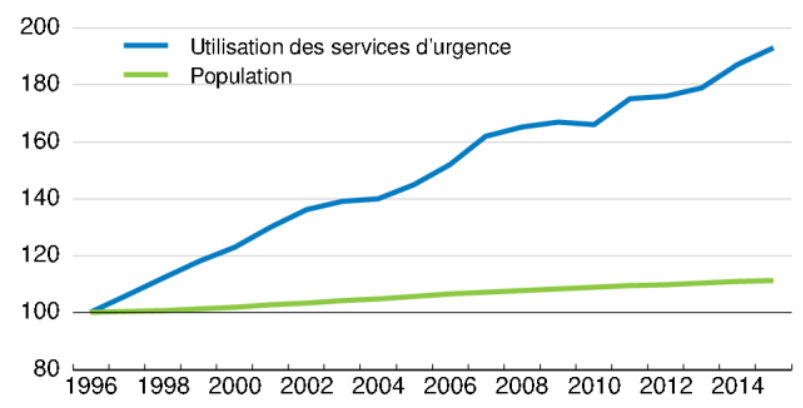

1. Ou année la plus proche.

2. Le champ retenu des dépenses institutionnelles diffère cependant de l'ensemble des dépenses de prévention en France, notamment lors des consultations ordinaires, qui représente selon la Drees 9.3 milliards d'euros en 2014 , soit $3.9 \%$ des dépenses de santé contre $2.0 \%$ en 2014 selon les données de l'OCDE.

3.

Source: OCDE (2017), Base de données des Statistiques de l'OCDE sur la santé ; Drees (2017), Les établissements de santé édition 2017 ; OCDE (2015), Panorama de la santé 2015 : Les indicateurs de l'OCDE, Éditions OCDE, Paris.

Le partage d'informations électroniques sur la santé, actuellement développé via le dossier médical partagé (DMP) ou d'autres dispositifs comme l'incitation des maisons de santé à disposer de systèmes d'information partagés entre maisons de santé, peut être renforcé. La mise en œuvre d'un système d'information dématérialisé en santé et de dossiers médicaux électroniques partagés progresse lentement. Les hôpitaux développent actuellement leurs systèmes informatiques (DGOS, 2016), et si le dossier pharmaceutique (DP) est répandu dans les pharmacies, la mise en œuvre des dossiers médicaux partagés (DMP) destinés à favoriser la prévention, la qualité, la continuité et la prise en charge coordonnée des patients prévue pour 2014 a pris du retard, avec seulement 600000 dossiers fin 2015. Plus généralement, il y a quelques années du moins, la France se plaçait derrière de nombreux pays européens en ce qui concerne l'adoption des dossiers médicaux électroniques personnels et l'échange d'informations entre professionnels (Commission européenne, 2013a), et elle n'est que peu apte à adopter des technologies électroniques (OCDE, 2017a). Un numéro de téléphone unique a été mis en place pour coordonner les services en dehors des horaires normaux depuis 2015, mais les progrès effectués ne sont que partiels. La stratégie nationale e-santé 2016-20 prévoit de donner la priorité à l'investissement dans les outils de 
coordination des prestataires de soins et au développement de solutions informatiques intégrées qui simplifieraient les procédures pour les patients et l'application des directives médicales pour les professionnels de santé.

Une meilleure utilisation des données disponibles sur la santé et le développement des dossiers électroniques pourraient améliorer la qualité des services et réduire les coûts administratifs. Un recours accru aux rapports de performances inciterait les prestataires à se concentrer sur des aspects de la qualité qui donneraient lieu à de véritables améliorations des résultats des patients. Les données administratives disponibles pourraient être davantage utilisées pour mener des évaluations médicoéconomiques ex post supplémentaires, notamment par des institutions indépendantes et des chercheurs, ou pour améliorer la détection des abus et des fraudes. A plus long terme, les dossiers électroniques utilisés dans les soins primaires pourraient inclure des informations utiles sur les pathologies et les prises en charge, afin d'améliorer les évaluations dont celles concernant les pratiques. Il serait également nécessaire de former les experts publics et indépendants, en exploitation des données notamment, l'exploitation des données à des fins administratives étant limitée à ce jour (Cour des comptes, 2016c). En outre, la HAS, qui développe des recommandations de bonnes pratiques de soins, se concentre sur les essais cliniques et le développement d'évaluations ex post des stratégies thérapeutiques, et des analyses complémentaires des données pourraient contribuer à adapter ses recommandations. De telles informations peuvent considérablement améliorer la formation continue des médecins et garantir l'indépendance vis-à-vis de l'industrie pharmaceutique mais les contraintes budgétaires de la HAS ne lui ont pas permis de mettre en place les structures nécessaires à ce travail.

La réforme des soins primaires et des hôpitaux contribuerait à régler les défauts de coordination. Par exemple, les urgences sont souvent utilisées pour des soins de routine, qui pourraient être pris en charge par un médecin généraliste. Elles sont perçues comme des "guichets uniques » accessibles, $55 \%$ des patients ayant signalé des difficultés d'accès aux médecins généralistes en dehors des horaires normaux en 2011 (Berchet, 2015 ; Berchet et Nader, 2016). Un numéro d'appel unique national a été mis en place en 2015 pour coordonner les services de santé en dehors des heures de travail. De nombreux progrès demeurent possibles. La mise en place d'une garde en dehors des horaires normaux dans la médecine de ville, dans le cadre de nouvelles formes d'organisation - comme les maisons médicales de garde - par exemple, et une sensibilisation accrue du public et des médecins sont essentielles. Toutefois, il faudra également inciter les hôpitaux à restreindre l'activité de leurs services d'urgences ou à en réduire la taille, puisque les tarifs des urgences sont déterminés par leur fréquentation passée et actuelle, indépendamment des pathologies prises en charge (Cour des comptes, 2014a). Au minimum, la participation financière des assurés devrait être modulée en fonction des soins.

La formation continue des professionnels des soins primaires et hospitaliers, qui améliore également les pratiques de travail, s'est renforcée dans le cadre de la loi de modernisation du système de santé de 2016. Depuis 2009, la formation continue est obligatoire pour les professionnels de santé (tous les trois ans), mais aucune véritable sanction n'est prévue en cas de non-respect de cette obligation. La formation s'inscrit dans le cadre d'un dispositif simple visant tous les professionnels de santé. Toutefois, la qualité des programmes de formation est insuffisamment contrôlée, le financement est limité à moins de $0.01 \%$ du PIB (Drees, 2016b), et le taux d'utilisation peut être amélioré : seul un quart de l'ensemble du personnel médical a entrepris une formation en 2014 (Deumie et al., 2014). Une formation commune complémentaire tout au long de la carrière permettrait une meilleure coordination des prestataires de soins et pourrait les aider à s'adapter aux meilleures pratiques en matière de parcours de soins et de prescriptions (voir plus bas). 


\section{Adapter le réseau de soins primaires et son système de rémunération}

La rémunération à l'acte demeure la principale source de revenus des prestataires de soins de ville, bien que la part du paiement à la performance et de la capitation tendent à s'accroître. A l'instar de la faiblesse du reste à charge, cela peut se refléter dans la consommation très élevée par habitant de médicaments sur ordonnance en France et la faible confiance du public dans certaines mesures préventives comme les vaccins (Larson et al., 2016). Cela peut aussi augmenter les prescriptions et la consommation de médicaments, et les dépenses au titre des soins primaires (graphique 13, parties A et B), même si la baisse de la densité médicale face à une demande croissante de soins peut limiter ces effets (Albouy et Deprez, 2008). La rémunération à l'acte n'est vraisemblablement pas adaptée pour favoriser une prise en charge coordonnée des maladies chroniques et des patients nécessitant des soins proactifs et coordonnés mettant l'accent sur les aspects préventifs (OCDE, 2016c). Par ailleurs, les taux d'immunisation des enfants contre la rougeole sont relativement bas (partie C), et l'immunisation contre la grippe a diminué chez les personnes âgées et demeure bien inférieure à $40 \%$ dans certaines populations à risque, malgré un objectif officiel de $75 \%$ (partie D ; PLFSS, 2015).

\section{Graphique 13. Résultats de certains soins primaires}

\section{A. Dépenses medicales en volume} Indice, $2005=100$

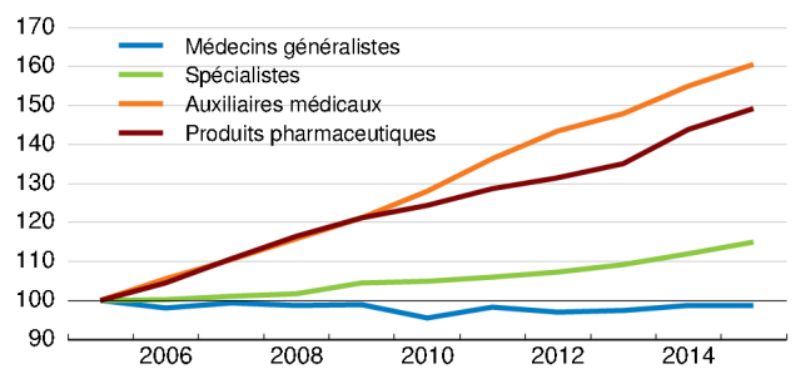

C. Immunisation contre la rougeole, 2013 En pourcentage des enfants

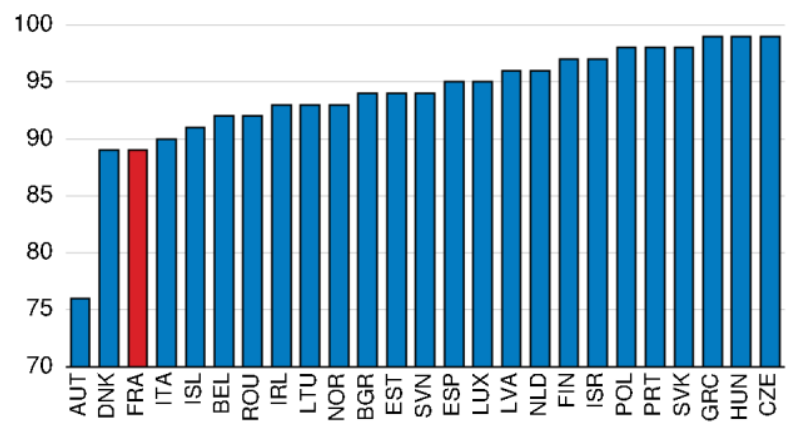

B. Consommation d'antibiotiques de seconde intention, $2013^{1}$ Doses quotidiennes définies pour 1000 habitants par jour

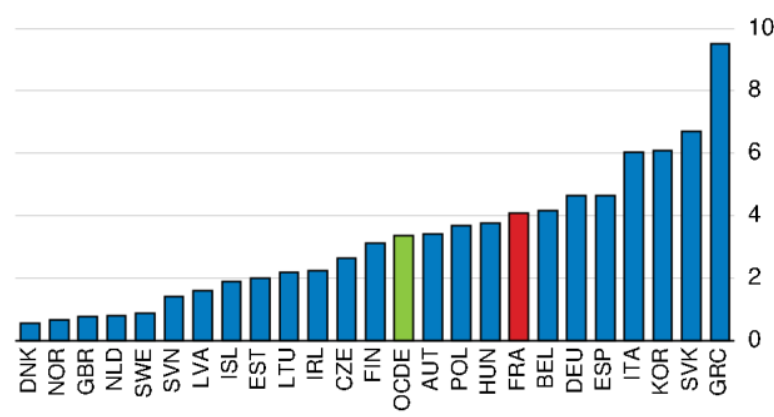

D. Immunisation contre la grippe En pourcentage de la population âgée de 65 ans et plus

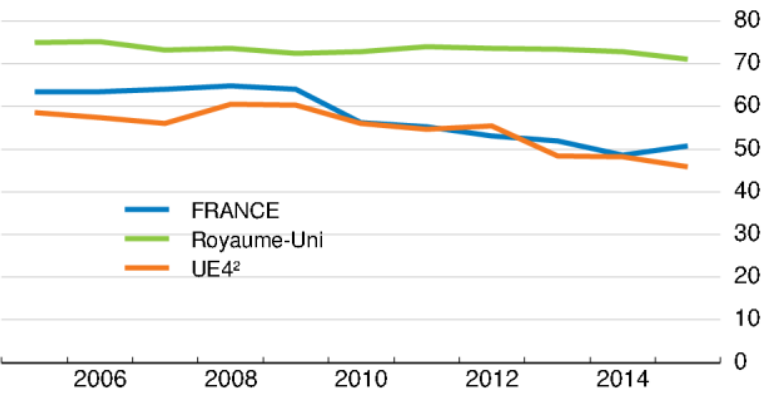

1. Ou dernière année disponible.

2. UE4 correspond à la moyenne de l'Allemagne, du Danemark, de l'Espagne et de l'Italie.

Source : OCDE (2017), Base de données des Statistiques de l'OCDE sur la santé ; Drees (2016), Portrait des professionnels de santé - Édition 2016 ; Organisation mondiale de la santé, Europe (2017), base de données CISID (http ://data.euro.who.int/CISID) ; Drees (2017), L'état de santé de la population en France - Édition 2017.

Les médecins généralistes sont souvent le premier interlocuteur dans le système de santé. Les autorités ont renforcé leur rôle de filtre en 2004 en incitant tous les patients à s'inscrire auprès d'un médecin traitant, tel que recommandé par l'OCDE (OCDE, 2000 ; Imai et al., 2000). Les patients participent davantage au coût s'ils ne déclarent pas un médecin traitant ou s'ils consultent un spécialiste sans avoir vu d'abord un généraliste. En outre, le taux de remboursement légal des consultations « hors du 
parcours de soins coordonné »a été progressivement réduit pour atteindre $30 \%$ en 2009 (sauf pour les patients consultant des gynécologues, des ophtalmologues et les jeunes en psychiatrie). Suite à cette réforme, l'accès direct aux spécialistes a rapidement diminué, passant à moins de $9 \%$ du total des consultations des spécialistes en 2014.

Il existe d'importantes disparités locales en termes de densités des professionnels de santé, et l'offre de soins primaires pourrait être mieux adaptée aux besoins locaux. Selon les prévisions, le nombre de médecins généralistes par habitant devrait passer temporairement sous la moyenne actuelle de l'OCDE vers 2025, en raison de leur pyramide des âges défavorable et des restrictions historiquement strictes du nombre d'étudiants en médecine (graphique 14, parties A et B ; Drees, 2016b ; Bachelet et Anguis, 2017). Cela pourrait éventuellement aggraver les pénuries locales (hors développement du cumul emploiretraite ou hausse des flux de médecins étrangers), les médecins âgés étant majoritaires dans les zones rurales et les quartiers pauvres, où l'accès est déjà limité (partie C).

\section{Graphique 14. Densité des médecins et rémunérations}

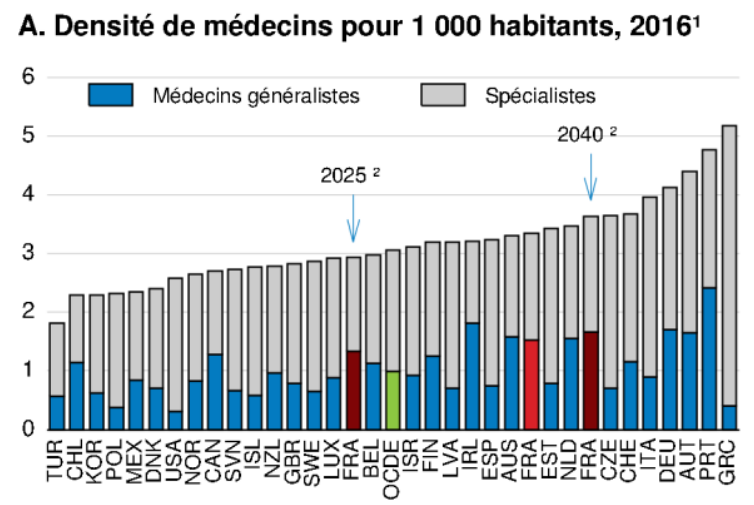

\section{B. Structure démographique des médecins, \%}

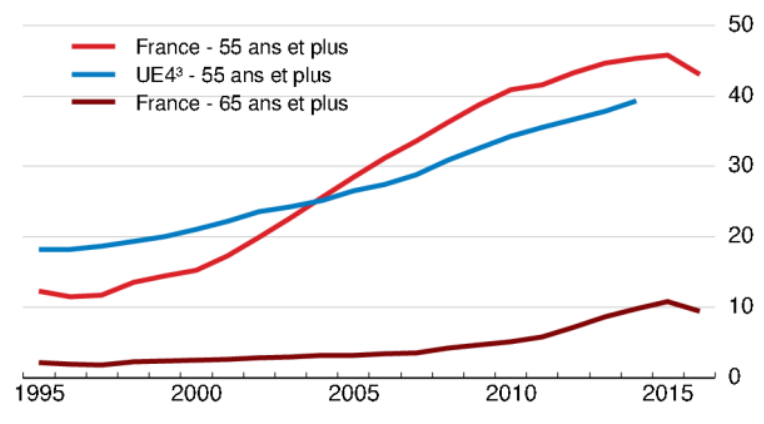

C. Disparités géographiques en termes d'accès aux médecins

Par rapport au niveau moyen d'accessibilité, 2013-14

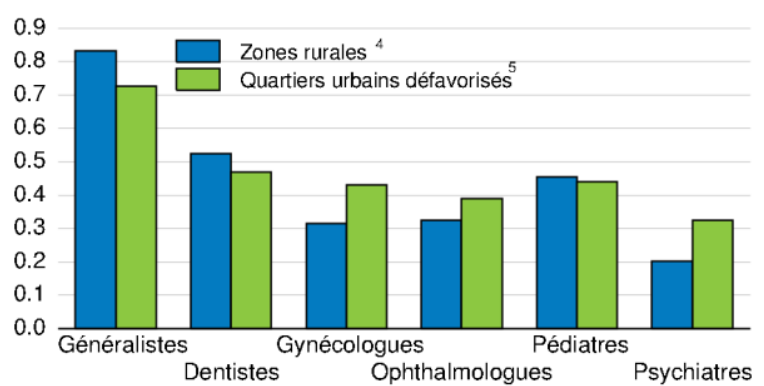

\section{Rémunérations des médecins généralistes} autres qu'à l'acte

En pourcentage des dépenses déclarées à la CNAMTS

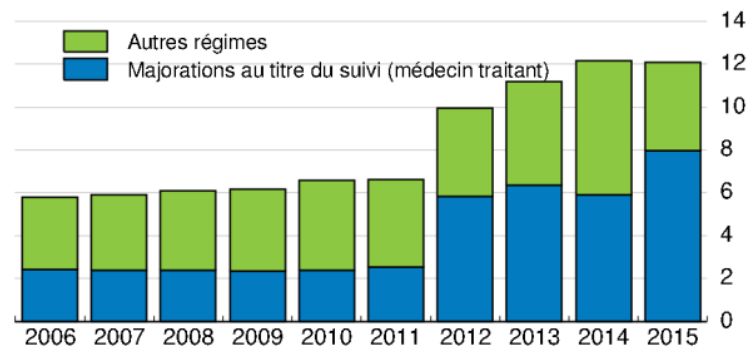

1. Ou dernière année disponible.

2. Projection de la Drees (2016), scénario central.

3. UE4 correspond à la moyenne de l'Allemagne, du Danemark, de l'Espagne et de l'Italie.

4. Ratio de l'indice d'accessibilité dans les zones rurales sur le même indice à l'échelle de la France. Les zones rurales sont pondérées en fonction de leur population en 2011.

5. Ratio de la densité par habitant dans les Quartiers prioritaires de la ville sur la densité en France métropolitaine.

Source: OCDE (2017), Base de données des Statistiques de l'OCDE sur la santé ; Drees (2016), Portrait des professionnels de santé - Édition 2016 ; Calculs de l'OCDE, d'après ONPV (2016), "L'offre de soins dans les quartiers prioritaires de la politique de la ville, en 2014 ", Rapport annuel de l'Observatoire national de la politique de la ville 2015 ; Vergier, N. (2016), "Accessibilité aux professionnels de santé libéraux: des disparités géographiques variables selon les conditions tarifaires ", Etudes et Résultats, $n^{\circ} 970$, Drees ; Brutel, C. et D. Levy (2011), “Le nouveau zonage en aires urbaines de 2010 », Insee Première, $\mathrm{n}^{\circ} 1374$; Drees (2016), Les dépenses de santé en 2015 - Résultats des comptes de la santé. 
Les disparités géographiques sont notamment prononcées pour les spécialistes et le personnel paramédical indépendant, puisque la plupart des spécialistes sont libres de fixer leurs tarifs et ont tendance à se concentrer dans les zones urbaines plus aisées où les ménages peuvent se permettre ces frais supplémentaires (Vergier, 2016; ONPV, 2016). En effet, les ménages des quartiers défavorisés avaient deux fois moins de chances d'être titulaires d'une assurance complémentaire que la population générale en 2012 (ONZUS, 2015), malgré les dispositifs CMU-C et ACS, et cela aurait pu effectivement limiter leur capacité à payer les dépassements d'honoraires (qui sont par ailleurs interdits envers ces patients). En outre, les dispositifs CMU-C et ACS limitent les honoraires des médecins et exonèrent les ménages du ticket modérateur, et les résultats de nombreux tests de discrimination indiquent que les ménages bénéficiaires de la CMU se voient fréquemment refuser des rendez-vous médicaux, notamment par les spécialistes pratiquant des dépassements d'honoraires (Baudis, 2014 ; Desprès et al., 2009).

Les autorités ont fortement augmenté le nombre de places de formation de médecins généralistes, et ont mis en place des incitations supplémentaires relatives à leur installation dans des régions moins bien desservies en médecins. La France, comme d'autres pays de l'OCDE, ne restreint pas la liberté d'installation des médecins (Ono et al., 2014). Depuis 2010, les étudiants en médecine peuvent recevoir des subventions mensuelles dans le cadre d'un contrat d'engagement de service public (CESP) s'ils s'engagent à exercer pendant au moins deux ans dans des régions en sous-densité. En outre, depuis 2012, les autorités garantissent un revenu annuel aux médecins généralistes récemment diplômés pendant les deux premières années suivant l'installation de leur cabinet dans des régions rurales et en sous- densité. La convention médicale de 2016 renforce ces dispositifs en mettant en place par exemple un contrat d'aide à l'installation des médecins. En outre, chaque étudiant en médecine doit consacrer au moins un de ses six semestres de formation dans un cabinet de médecine générale à compter de 2017.

Ces mesures vont dans le bon sens. Toutefois, le CESP n'a attribué des bourses qu'à seulement 236 étudiants, dont 172 en médecine générale, parmi les 7700 nouveaux étudiants en médecine en 2016 (Anguis, 2017), et les prévisions démographiques et la planification des capacités de formation s'effectuent principalement au niveau national, à l'aide de quotas relatifs au nombre d'étudiants admis dans les programmes de médecine, pharmacie, odontologie, maïeutique (et kinésithérapie pour partie) ou d'autres domaines tels que les soins infirmiers ou l'orthophonie (numerus clausus). En France, les universités publiques assurent l'éducation et la formation des professionels de santé qui ont ensuite une liberté d'installation. La définition centralisée du numerus clausus régional applicable aux médecins et les épreuves classantes nationales qui permettent d'attribuer une spécialité et une faculté à chaque étudiant sont coordonnés avec les internats pour mieux prendre en compte les besoins locaux. En effet, la localisation des universités conditionne en partie les premières installations des médecins généralistes (ONDP, 2014 ; Golfouse et Pheng, 2015). Ceci pourrait influer sur leur installation future. Les numerus clausus régionaux devraient être plus étroitement liés à la capacité d'enseignement des universités régionales et aux besoins prévus, tandis que la formation initiale devrait inclure davantage de formations et stages communs pour les futurs médecins généralistes, infirmiers et pharmaciens, afin d'améliorer la coordination à moyen terme. Ils pourraient être complétés par une hausse des paiements à la capitation dans les régions moins bien desservies, ce qui pourrait compenser leur moindre potentiel de dépassements d'honoraires, et les inégalités sur le plan de la santé de la population et relatives à leurs propres conditions de travail.

Les médecins conventionnés secteur 1 sont soumis à des tarifs réglementés, et les autres, conventionnés secteur 2, peuvent pratiquer des dépassements d'honoraires. Pour ceux qui ne pratiquent que peu de dépassements, ils ont la possibilité de signer un contrat avec l'assurance maladie afin de bénéficier d'une rémunération supplémentaire sur la base d'un paiement à la performance. La poursuite du développement des paiements à la performance peut encourager les gains d'efficience et des traitements plus ciblés. Toutefois, ils ne représentent encore qu'une petite part des revenus des médecins généralistes libéraux (graphique 14, partie D). Le ministère de la Santé prévoit des incitations complémentaires pour améliorer l'accès aux soins de santé, limiter les dépassements d'honoraires et 
encourager l'informatisation des cabinets médicaux, mais les dispositifs de rémunération aux résultats pourraient également être étendus. Des paiements forfaitaires pourraient également être mis en place pour les infirmiers, les sages-femmes et les kinésithérapeutes, les prescriptions et les dépenses ambulatoires augmentant rapidement dans ces activités de soins, croissance tirée pour large partie par des prescriptions émanant de prescripteurs hospitaliers (graphique 13, partie A). En effet, ces prescriptions font l'objet de restrictions moindres que d'autres dépenses médicales. L'établissement de paiements forfaitaires par patient spécifiques pour le suivi des maladies chroniques ou pour les services à long terme serait utile.

Le plafonnement des dépassements d'honoraires des spécialistes permettrait également de faire des économies et améliorerait l'accès aux soins pour l'ensemble des groupes de revenu. Cela pourrait également en partie affecter l'offre de nouveaux spécialistes, et un tel changement nécessiterait de procéder avec précaution. Quelque $45 \%$ des spécialistes sont autorisés à pratiquer des dépassements d'honoraires, sauf si les patients bénéficient de la CMU-C et de l'ACS. Les dépassements d'honoraires ont augmenté rapidement, et représentaient $18 \%$ de la rémunération de l'ensemble des spécialistes en 2015 (soit $0.1 \%$ du PIB), contre moins de $1 \%$ pour les majorations et la rémunération aux résultats (Drees, 2016c). Les autorités ont proposé à certains spécialistes des contrats facultatifs leur garantissant notamment une prise en charge partielle de leurs cotisations sociales en contrepartie d'une limitation de leurs dépassements d'honoraires, mais peu ont choisi d'en bénéficier. Depuis 2015, les organismes complémentaires doivent plafonner le remboursement des dépassements d'honoraires (à hauteur de $125 \%$ du tarif réglementé) afin de bénéficier de certains allègements fiscaux. Toutefois, le nombre de consultations de spécialistes a augmenté relativement vite (graphique 13, partie A), et les dépassements d'honoraires concernent surtout des formes de soins et des zones géographiques spécifiques, ce qui engendre des inégalités. La mise en place d'un système de tarification ajustable et des majorations pour l'ensemble des professionnels de santé avec un plafond budgétaire comme en Allemagne pourrait être plus efficace pour ralentir la croissance des dépenses et la demande induite (Bozio et Dormont, 2016) ; une autre solution consisterait à plafonner le taux des dépassements d'honoraires par acte.

Des innovations sur le plan de l'organisation permettraient de remédier aux pénuries et d'améliorer la coordination des prestataires. Les généralistes français consacrent énormément de temps aux tâches non médicales (Jakoubovitch et al., 2012). Bien que la majorité des médecins et près de $40 \%$ du personnel infirmier travaillent dans des cabinets de groupe (Drees, 2016b), le développement de structures permettant de consulter des médecins non hospitaliers locaux et d'autres professionnels de santé en dehors des horaires normaux pourrait favoriser une meilleure couverture géographique, une disponibilité $24 \mathrm{~h} / 24$ et la continuité des traitements. Un système de maisons de santé pluridisciplinaires (MSP) a été mis en place en 2007 et renforcé par la loi de modernisation de notre système de santé de 2016. Il permet aux médecins ou autres professionnels de santé d'exercer dans des cabinets de groupe tout en restant indépendants. Les MSP et les centres de santé semblent avoir amélioré les conditions de travail et l'accessibilité pour les patients, et avoir engendré certains gains d'efficience (Mousquès et Daniel, 2015a et 2015b).

Cependant, l'exercice regroupé pluriprofessionnel peut encore être développé (HCAAM, 2014). Des aides publiques encouragent le développement des MSP. En outre, la composition des professionnels de santé, l'investissement financier dans les MSP et les cabinets communs de médecins généralistes sont limités par différentes contraintes juridiques et critères de propriété (OCDE, 2016b et c). L'incertitude au sujet de la rémunération des services communs et des paiements globaux a également été un obstacle, et l'accord interprofessionnel d'avril 2017 pourrait faciliter la collaboration entre les professionnels de santé. Cependant, une simplification supplémentaire des structures et des exigences administratives pourrait également aider (FFMPS, 2017), ainsi que l'abaissement des restrictions de l'autoréglementation (voir ci-dessous) et l'augmentation du partage de responsabilités entre médecins, 
infirmières et autres professionnels. Le gouvernement envisage de faciliter le financement des équipes pluriprofessionelles en 2018 et de doubler le nombre de MSPs d'ici 2022.

La redéfinition des missions des infirmiers et pharmaciens en tant que professionnels de santé et la conception de solutions d'e-santé améliorerait l'efficience. La législation nationale actuelle définit en termes précis ce que chaque profession de santé peut ou ne peut pas faire (Delamaire et Lafortune, 2010), et les incitations financières à la définition de nouvelles missions pour les prestataires sont faibles. Par exemple, les activités médicales des infirmiers restent limitées, malgré les protocoles de coopération autorisés par la loi Hôpital Patients Santé Territoires en 2009. De plus, le code de déontologie professionnelle de l'Ordre national des infirmiers a restreint encore la concurrence et la taille des cabinets (Autorité de la concurrence, 2016a). Bien que la loi de modernisation de notre système de santé de 2016 ait quelque peu élargi le rôle des sages-femmes, une définition plus générale du champ d'activité des différentes professions, par exemple en termes de "missions» générales plutôt que de tâches/actes spécifiques, favoriserait une plus grande flexibilité. Par exemple, les véhicules de tourisme avec chauffeur pourraient être autorisés à transporter les patients autonomes et à compléter les services des chauffeurs de taxi, et les optométristes pourraient être autorisés à prescrire des appareils médicaux, tels que les lunettes (IGF, 2013).

Le développement de la télémédecine pour mettre en relation les patients et les médecins à distance lorsque c'est nécessaire pourrait permettre de faire des économies, d'améliorer le suivi des maladies chroniques et l'observance des traitements (Compagnon and Lopez, 2015). Cette prise en charge de la télémédecine par l'assurance maladie va être mise en place dès 2018. De même, la vente en ligne de médicaments non soumis à prescription demeure particulièrement restreinte (cela étant justifié par des raisons sanitaires), à l'instar de l'investissement dans les pharmacies, malgré un assouplissement partiel des réglementations en 2016 (Autorité de la concurrence, 2016b).

\section{Moderniser la gestion et les services hospitaliers}

La réduction des coûts hospitaliers devrait générer des économies importantes. Les dépenses hospitalières ont régulièrement augmenté pour s'établir à $4.4 \%$ du PIB en 2014 et $40 \%$ des dépenses actuelles de santé (OCDE, 2016d). Le nombre de lits d'hôpital par habitant est relativement élevé, et leur taux d'occupation est seulement moyen, et l'emploi hospitalier est supérieur à la moyenne de l'OCDE, notamment chez les employés administratifs et le personnel de soutien (graphique 15, parties A à C). Les établissements hospitaliers se composent d'établissements publics, d'établissements privés à but non lucratif et d'établissements privés à but lucratif. Leur taille moyenne est relativement réduite (partie D), et le nombre de petits établissements est élevé, malgré leur efficience relativement inférieure et leurs pratiques moins innovantes (Gobillon et Milcent, 2013 et 2016). D'après les données de l'OCDE, le coût moyen de certains actes chirurgicaux est supérieur de $23 \%$ au coût constaté dans d'autres pays européens (Koechlin et al., 2014).

La mise en place d'un système de paiement à l'activité (tarification à l'activité - T2A) a permis certaines améliorations. Comme dans de nombreux autres pays de l'OCDE (OCDE, 2016c) et selon les recommandations de l'OCDE (OCDE, 2000), un dispositif de rémunération fondé sur les groupes homogènes de séjours (GHS) a été progressivement mis en place entre 2004 et 2008. Il remplace l'ancien système de subventions globales et de forfaits journaliers. Il est complété par des rémunérations fondées sur le volume d'activité, comme la rémunération des services d'urgence et des traitements innovants, ainsi que des paiements complémentaires, comme les financements de l'enseignement, la recherche et l'innovation, la maintenance des services d'urgence, la prévention et l'admission des patients à risque et modestes. La réforme des GHS s'est traduite par une moindre variabilité des coûts d'un établissement à l'autre (Milcent, 2016a) et une hausse de l'activité chirurgicale des hôpitaux publics exposés à la concurrence du secteur privé (Choné et al., 2014). 
Graphique 15. Le secteur hospitalier, $2015^{1}$

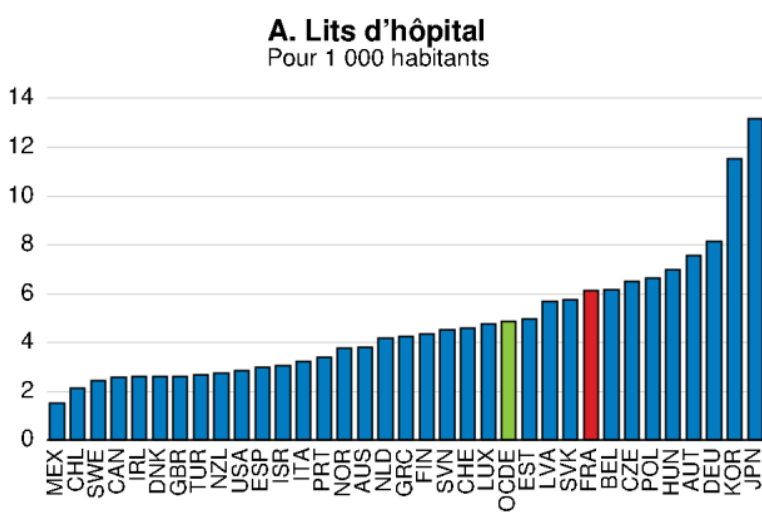

C. Personnel non hospitalier employé à l'hôpital En pourcentage des effectifs totaux

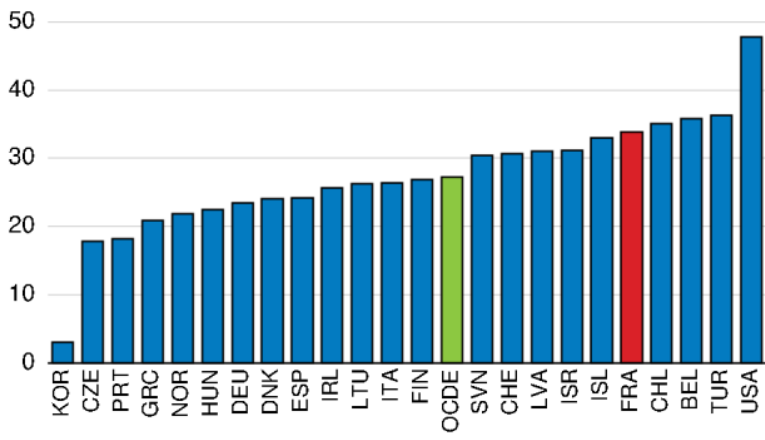

B. Emploi hospitalier Pour 1000 habitants

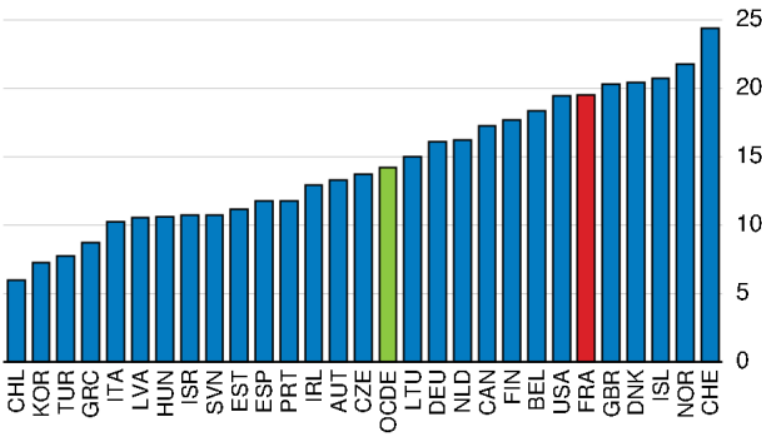

D. Taille moyenne des établissements hospitaliers Nombre de lits

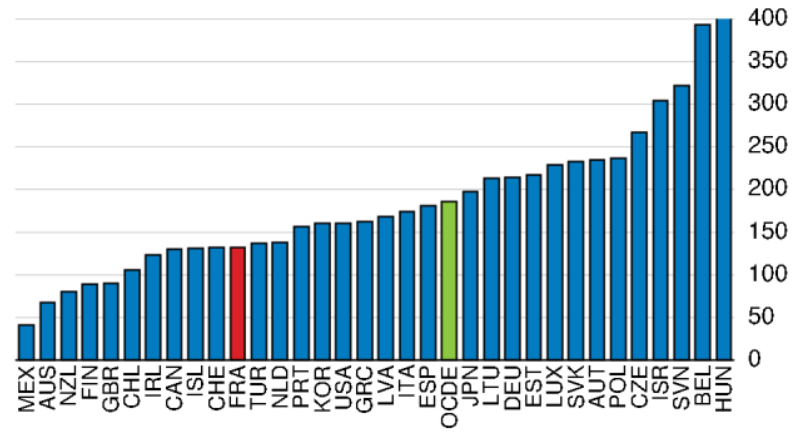

1. Ou année la plus proche.

Source : OCDE (2017), Base de données des Statistiques de l'OCDE sur la santé.

\section{Encadré 3. Paiement des hôpitaux et système des groupes homogènes de séjours (GHS)}

En France, les hôpitaux de court séjour, à l'exclusion des hôpitaux psychiatriques, sont financés par un système de paiement fondé sur les groupes homogènes de séjours (GHS). Ce système alloue des financements aux hôpitaux en fonction du nombre de séjours, des pathologies, des patients et des tarifs. Le régime d'assurance maladie obligatoire affecte chaque séjour à un GHS. Les hôpitaux perçoivent ensuite une somme qui dépend du nombre de patients dans chaque GHS et des tarifs associés.

Le ministère de la Santé fixe les tarifs des GHS. Ces tarifs reflètent les coûts moyens de traitement d'un GHS dans un échantillon d'hôpitaux et le plafond budgétaire annuel global des hôpitaux (encadré 2). Les tarifs correspondent aux coûts moyens des traitements de chaque GHS, réajustés pour s'assurer que le plafond budgétaire global annuel est atteint. Ils visent également à encourager certaines activités telles que la chirurgie ambulatoire. Ainsi, les tarifs ne reflètent pas forcément les variations des coûts hospitaliers moyens. En outre, des tarifs inférieurs sont appliqués aux journées de soins supérieures et inférieures aux seuils de durée standard dans chaque groupe de GHS. Les tarifs varient également entre les établissements publics et à but non lucratif et les établissements privés où les honoraires des médecins sont payés séparément.

Le système de GHS a été réformé à de nombreuses reprises depuis sa mise en place en 2004. En particulier, le nombre de GHS est passé de 700 en 2004 à quelque 2300 en 2009, et les seuils de durée ont été régulièrement ajustés. Par exemple, depuis 2010, certains courts séjours sont payés par une somme forfaitaire et un tarif à la journée. 
La rémunération diffère selon les hôpitaux. Les tarifs des GHS diffèrent entre hôpitaux publics et privés. Ils ne prennent pas en compte la rémunération des professionnels de santé dans les hôpitaux privés, ce qui ne permet pas une convergence des tarifs. En outre, les hôpitaux publics disposent d'un peu de marge pour ajuster les montants facturés aux organismes complémentaires et aux ménages comme les tarifs journaliers de prestation. Ces différences brouillent les incitations en lien avec les rémunérations publiques et ne garantissent pas l'équité pour les patients. Il serait préférable d'augmenter les majorations pour les cas difficiles afin d'éliminer progressivement ces disparités, car les paiements par GHS pourraient ne permettre qu'une réduction partielle de l'hétérogénéité entre les établissements (Milcent, 2016a ; Véran 2016).

Les paiements par GHS fondés sur les coûts moyens peuvent accroître les risques de sélection des patients, la spécialisation dans les procédures les plus rentables et réduire l'attention portée à la qualité au sein des groupes de GHS. Ils peuvent également inciter à maximiser les déclarations d'activité et conduire à exagérer les difficultés de chaque patient. En France, les groupes de GHS reflètent moins de la moitié de l'hétérogénéité des patients (Milcent, 2016b), et le phénomène de codage opportuniste (à savoir la déclaration de groupes de GHS plus avantageux que les services réellement fournis) semble considérable, comme aux États-Unis (Milcent, 2016a et 2016b; Dafny, 2005). En outre, comme dans les soins primaires, la structure de rémunération ne favorise pas vraiment la prévention et la coordination entre les prestataires de soins. En effet, le nombre d'hospitalisations est élevé, et la durée des séjours demeure relativement longue (graphique 16).

\section{Graphique 16. Utilisation des hôpitaux}

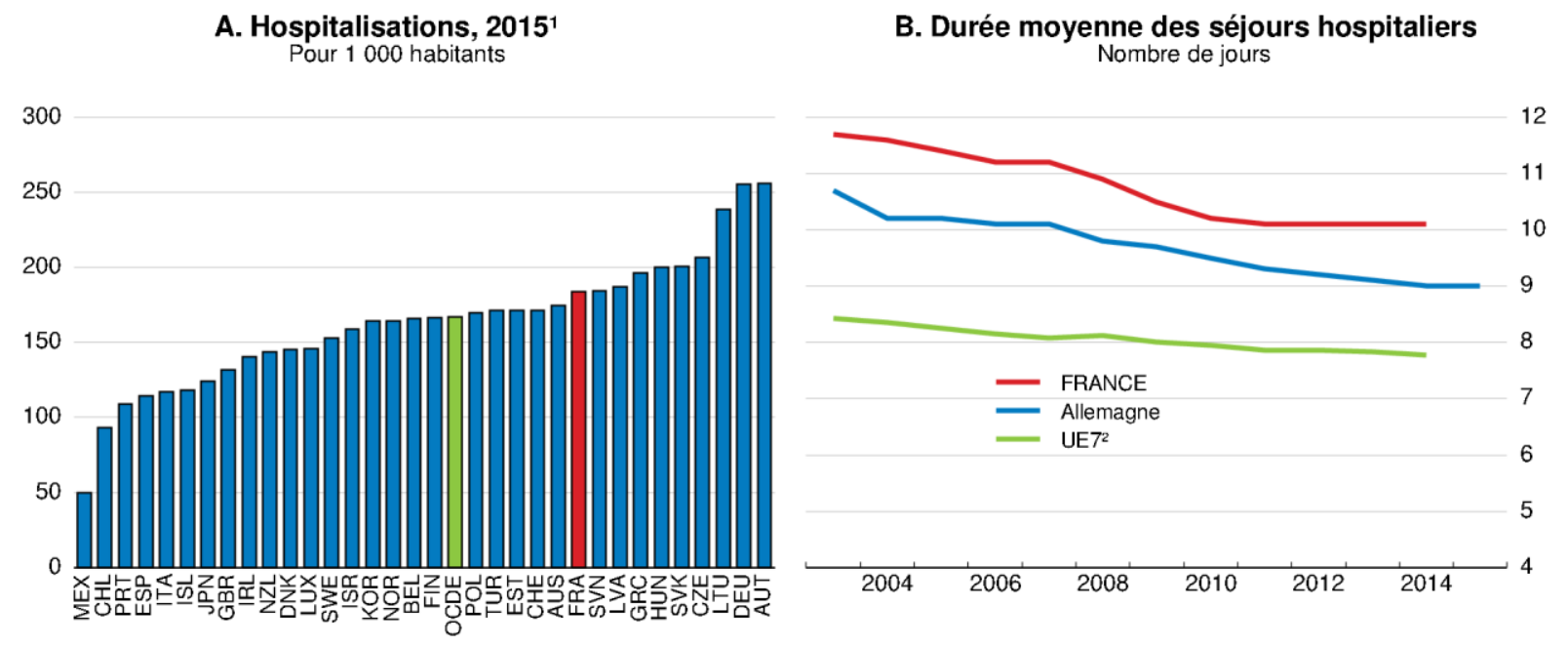

1. Ou année la plus proche.

2. UE7 correspond à la moyenne de l'Allemagne, l'Autriche, la Belgique, le Danemark, l'Espagne, l'Italie et le Portugal.

Source : OCDE (2017), Base de données des Statistiques de l'OCDE sur la santé.

Depuis 2012, les autorités ont pris des mesures complémentaires pour réformer le paiement des hôpitaux et réduire les incitations négatives des paiements par GHS. Des ajustements en fonction de la qualité des soins ont été testés et mis en œuvre, bien que leur montant demeure inférieur à $0.5 \%$ des dépenses médicales des hôpitaux participants. En 2017, les subventions aux équipements de soins aigus ont augmenté pour mieux tenir compte des coûts de maintenance fixes. En outre, les autorités prévoient de développer des paiements groupés pour les services de soins aigus et de réadaptation afin d'améliorer le parcours des patients atteints de maladies chroniques. Dans le même temps, la loi de 2016 garantit le financement d'hôpitaux de proximité, qui touchent des subventions spéciales depuis 2014, tout en ciblant leurs missions sur les soins primaires et le suivi des patients. Ces réformes peuvent améliorer la flexibilité du système de rémunération, mais elles nécessiteront que les ARS suivent de près les gains 
d'efficience des hôpitaux pour assurer l'efficacité des incitations d'augmentation de la productivité et éviter d'entraver la réorganisation des services hospitaliers, comme les rémunérations deviennent de plus en plus variables selon les hôpitaux.

La gestion des hôpitaux publics demeure difficile. Leur dette était d'environ 1.4\% du PIB en 2015 à la suite de programmes d'investissement massifs aux rendements variables du début des années 2000 (Cour des comptes, 2014b), et le secteur est souvent resté déficitaire, notamment en raison d'un service de la dette élevé (Drees, 2016d). La masse salariale représente $60 \%$ des dépenses publiques hospitalières et pourrait servir à ajuster les dépenses, mais les gestionnaires ont peu d'impact sur les décisions d'embauche, les carrières et les salaires des fonctionnaires et des médecins. Les indicateurs disponibles de la qualité de la gestion des ressources humaines apparaissent faibles dans les comparaisons internationales (Bloom et al., 2014). Par exemple, la semaine de travail de 35 heures et les hausses générales annoncées des salaires de base des fonctionnaires et la prise en compte des primes dans les droits à la retraite en 2016 ont augmenté les dépenses et les dettes à long terme. Dans le même temps, les primes accordées aux médecins ou infirmières acceptant des responsabilités de gestion ou médicales supplémentaires sont strictement limitées, et la responsabilité du personnel médical n'est engagée que de manière restreinte en ce qui concerne les effets de ses prescriptions sur les dépenses, que ce soit à l'hôpital ou en ville (Cour des comptes, 2016d).

Renforcer la gestion des ressources humaines améliorerait la prise en charge des patients et les conditions de travail. La rigidité de la gestion des ressources humaines, s'agissant notamment de la rémunération des médecins, a pu notamment entrainer une hausse du recours aux contrats temporaires avec des praticiens de santé indépendants, particulièrement coûteux (Véran, 2013), ce qui a potentiellement dégradé les résultats des patients (Bartel et al., 2014) et les conditions de travail (Coutrot et Davie, 2014). En outre, la gouvernance des hôpitaux universitaires est compliquée par les différentes affiliations de leur personnel aux universités, aux autres hôpitaux et aux centres de recherche, et au manque de coordination entre tous ces acteurs. Par exemple, les médecins peuvent cumuler des responsabilités d'enseignement, de soins et de recherche auprès de différents organismes publics.

Le renforcement de l'autonomie des gestionnaires publics des hôpitaux et l'amélioration de la coordination entre les établissements pourraient garantir un accès plus équitable aux soins hospitaliers. Les gestionnaires publics ont peu d'autonomie en ce qui concerne les décisions d'investissement, ce qui peut faire obstacle à une mise en œuvre efficace du système de GHS, puisque cela empêche la restructuration des hôpitaux publics et la réalisation des gains d'efficience potentiels (BruantBisson et al., 2012). Les interventions politiques locales dans la gestion des hôpitaux publics se sont également traduites par des sureffectifs et des inefficiences, notamment dans les régions où le chômage est élevé (Clark et Milcent, 2011 et 2015). Il pourrait sembler souhaitable que les hommes politiques locaux, n'aient pas le droit de gérer les conseils de surveillance des hôpitaux ou d'intervenir dans la nomination de leurs dirigeants. Dans le même temps, la mise en place des groupements hospitaliers de territoire encourage la coordination entre les hôpitaux, afin de limiter les disparités géographiques en termes d'accès aux services innovants (Drees, 2016d).

La loi de modernisation de notre système de santé de 2016 cherche à accroître les synergies entre les hôpitaux publics. Les groupements hospitaliers de territoire devraient permettre la création de services médicaux communs, notamment pour les marchés publics, et certains gains d'efficience. En outre, la généralisation du dispositif d'incitations financières à l'amélioration de la qualité en 2016 pour les établissements de santé du champ «médecine-chirurgie-obstétrique», élargie en 2017 au champ des établissements de soins de suite et de réadaptation, est bienvenue : à l'hôpital, l'excellence (résultats obtenus) et l'effort (évolution observée) sont récompensés selon des indicateurs nationaux de qualité et de sécurité des soins (par exemple ceux relatifs aux infections associées aux soins, à des prises en charge spécifiques, à la satisfaction des patients hospitalisés), des indicateurs issus du programme national Hôpital 
numérique (par exemple, ceux relatifs au dossier patient informatisé, à la prescription électronique) et la certification HAS des établissements de santé. Le montant complémentaire maximal est égal à $0.6 \%$ de leurs activités (plafonné à 500000 euros par an). Toutefois, beaucoup dépendra de leur mise en œuvre, et le développement d'une coopération efficace nécessitera que les paiements par GHS et le récent dispositif de financement des hôpitaux de proximité continuent d'évoluer. Des recherches économétriques indépendantes utilisant des données au niveau des hôpitaux aideraient également à identifier les meilleures pratiques et les gains potentiels d'efficacité.

\section{Maîtriser les dépenses pharmaceutiques}

La croissance des dépenses pharmaceutiques en part du PIB a été maîtrisée avec succès depuis le milieu des années 2000 en ce qui concerne les soins de ville, mais demeure bien plus élevée que dans d'autres pays de la zone euro (graphique 17, partie A). Le recours accru aux médicaments génériques et l'expiration des brevets de certains médicaments très utilisés ont contribué à la baisse des dépenses pharmaceutiques constatée depuis 2014, rendue possible par le maintien des prix réglementés à un bas niveau. Le financement de traitements coûteux n'a cependant pas empêché la hausse continue des consommations en volume (partie B). À plus long terme, la hausse des maladies chroniques et l'arrivée sur le marché de nouveaux médicaments innovants mais coûteux augmentera la pression sur les dépenses pharmaceutiques (OCDE, 2015b). En outre, le volume de consommation de médicaments pose des problèmes de santé considérables chez les personnes âgées (Jardin et al., 2012). Le recours accru aux évaluations médico-économiques des médicaments, la hausse de la part des médicaments génériques et la réduction du volume des prescriptions pourraient permettre de réaliser des économies supplémentaires, d'améliorer les résultats de santé, de réduire le gaspillage de médicaments et de maintenir les incitations à l'innovation pharmaceutique.

\section{Graphique 17. Consommation et prix pharmaceutiques}

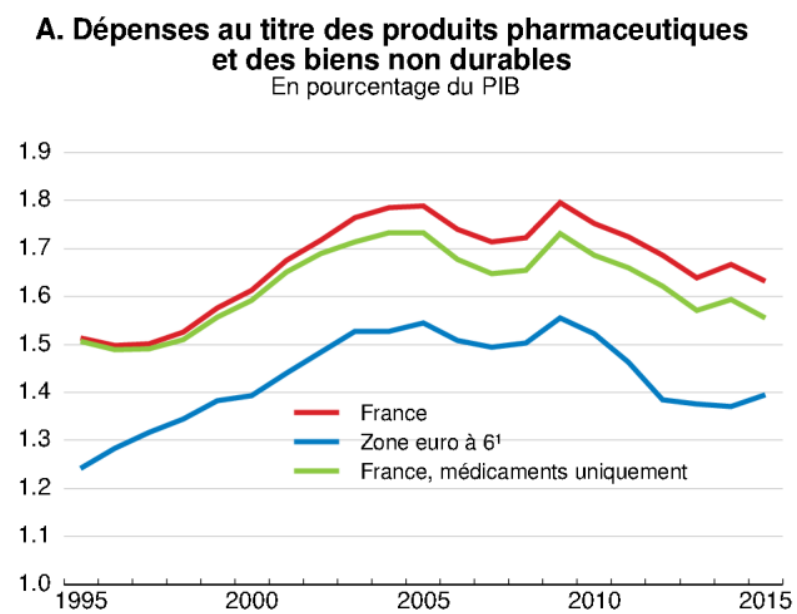
B. Prix et consommation de produits pharmaceutiques en France Indice, $2001=100$

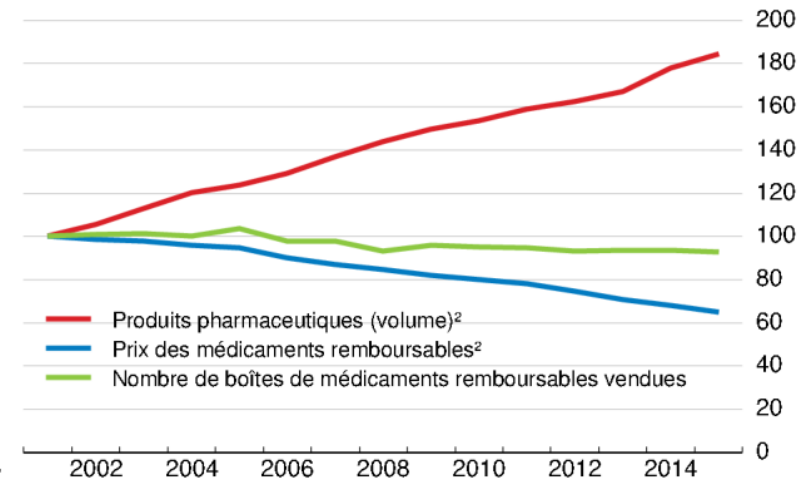

1. Correspond à la moyenne de l'Allemagne, l'Autriche, l'Espagne, l'Italie, les Pays-Bas et le Portugal.

2. À qualité constante, à l'exclusion des nouveaux produits médicaux et des innovations. Les indices des prix de détail (définition nationale) incluent les remboursements des régimes d'assurance obligatoires.

Source: OCDE (2017), Base de données des Statistiques de l'OCDE sur la santé ; Drees (2016), Les dépenses de santé en 2015 Résultats des comptes de la santé, Drees. Comité économique des produits de santé (2015), Rapport d'activité 2015.

Les prix réglementés, les marges, les taxes spécifiques et les taux de remboursement influencent la consommation de la plupart des produits pharmaceutiques. Les ménages peuvent acheter certains médicaments remboursables sans ordonnance, mais l'assurance maladie ne prend pas en charge ces dépenses, ni les médicaments non remboursables. Les médicaments sur ordonnance et les médicaments remboursables représentent plus de 87 et $90 \%$ du marché de détail, respectivement (en valeur) 
(graphique 18, partie A ; Drees, 2016c). Neuf taxes spécifiques sont appliquées aux laboratoires pharmaceutiques, dont trois visent à réduire la consommation excessive (Baulinet et al., 2012). En particulier, comme en Belgique et en Italie, des clauses de volume spécifiques sont automatiquement appliquées aux laboratoires pharmaceutiques pour compenser les dépassements budgétaires sur certains médicaments. Elles ont augmenté en 2015 et 2017, et limitent la part des revenus des ventes de détail revenant aux laboratoires (partie B), mais elles ne luttent pas directement contre les prescriptions excessives dans les soins ambulatoires. Des taxes spécifiques sont également prélevées sur les grossistes et les pharmaciens. En revanche, une réduction du taux de TVA sur les produits pharmaceutiques encourage la consommation. Les taux réduits sur les médicaments devraient être progressivement supprimés.

\section{Graphique 18. Ventes au détail de produits pharmaceutiques}
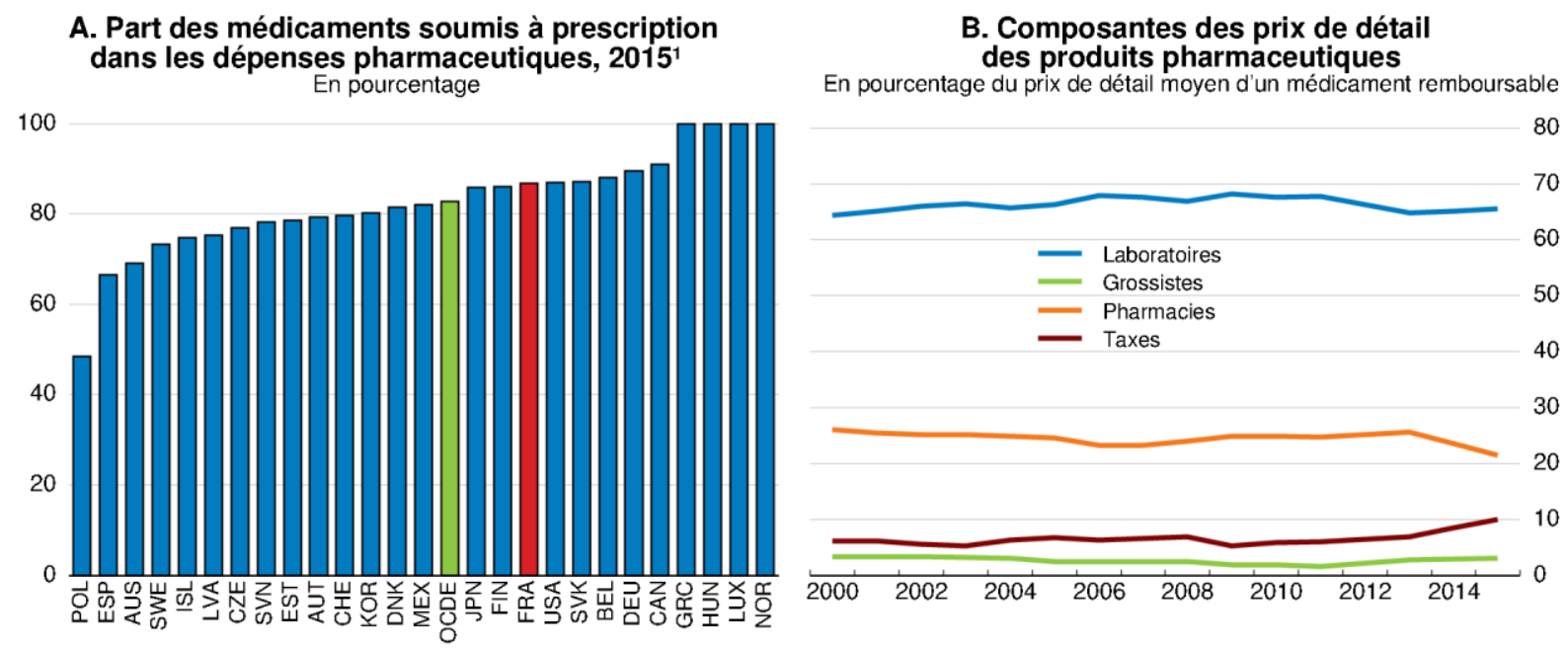

1. Ou dernière année disponible.

Source: OCDE (2017), Base de données des Statistiques de l'OCDE sur la santé ; Ecosante.fr ; et LEEM (2013 à 2016), Les Entreprises du médicament en France.

En France, les taux de remboursement et les prix de détail des produits pharmaceutiques sont liés à leur efficacité médicale. Plus précisément, le taux de remboursement dépend de l'efficacité du médicament et de la gravité de la maladie et le prix du médicament dépend de l'efficacité relative en comparaison avec les autres produits déjà existant sur le marché. Le ministère de la Santé autorise le remboursement des médicaments en fonction de l'avis de la Haute autorité de santé. La HAS se fonde à la fois sur une mesure du ratio coût-efficacité et son évaluation de la valeur ajoutée thérapeutique pour faire ses recommandations. Avant la décision finale de remboursement, le CEPS (Comité économique des produits de santé) négocie les prix notamment sur la base de l'évaluation faite par la HAS. Le CEPS prend également en compte la situation du marché, la taille de la population cible et les données disponibles du marché international. Par ailleurs, l'UNCAM fixe les taux de remboursement sur la base de l'évaluation de la HAS qui tient compte de la gravité des pathologies traitées. Le cadre réglementaire prévoit une réévaluation obligatoire des médicaments par la HAS tous les cinq ans, qui peut conduire dans certains cas à la suppression de remboursement. En même temps, une réévaluation fréquente des effets de la plupart des médicaments individuels et de leurs équivalents aide à mieux cibler les remboursements.

Le renforcement du lien entre les évaluations des médicaments, leur remboursement et leur prix de détail limiterait les usages inefficients. Les taux de remboursement de l'assurance maladie pourraient être plus étroitement liés aux résultats des mesures de coût-efficacité. Les médicaments peu efficaces affichent un taux de remboursement moyen de $38 \%$, bien supérieur au taux de remboursement recommandé de $15 \%$ (Polton, 2015). En outre, les produits homéopathiques bénéficient d'un taux de 
remboursement de $30 \%$, bien que leur efficacité ne soit pas prouvée (OCDE, 2011b). Par ailleurs, certains médicaments innovants coûteux vendus par les hôpitaux (liste en sus) ne font pas l'objet d'une réévaluation automatique.

Un recours accru aux médicaments génériques permettrait de réduire encore les coûts. (graphique 19, partie A). Les autorités françaises définissent les médicaments auxquels les génériques peuvent se substituer sur la base du répertoire des médicaments génériques, plus restrictif que celui d'autres pays puisque le groupage se fait avec les spécialités de même principe actif, de même dosage et avec le même mode d'administration, au lieu du seul principe actif par exemple (Caby et Zafar, 2017). Les génériques ne sont disponibles que dans le cadre de leur inscription dans ce répertoire (la part des classes de génériques autorisées dans les médicaments prescrits n'était que de $43 \%$ en volume en 2015), alors que d'autres pays comme l'Allemagne ont permis une utilisation plus large des génériques en définissant des groupes différents non seulement pour les médicaments ayant des principes actifs identiques, mais aussi pour les produits ayant des effets thérapeutiques comparables. Par conséquent, en France, la part globale des génériques dans l'usage pharmaceutique est restée inférieure à un tiers en 2015, malgré un taux de substitution proche de $80 \%$ dans les classes de médicaments autorisées (partie B). Élargir la liste des médicaments que les génériques peuvent remplacer et, plus généralement, permettre une plus grande utilisation des génériques seraient utiles (Cour des comptes, 2014c). En effet, les autorités prévoient un développement limité de la liste des médicaments que les génériques peuvent remplacer (ministère des Affaires sociales, de la Santé et des Droits des femmes, 2015).

\section{Graphique 19. Consommation de génériques}

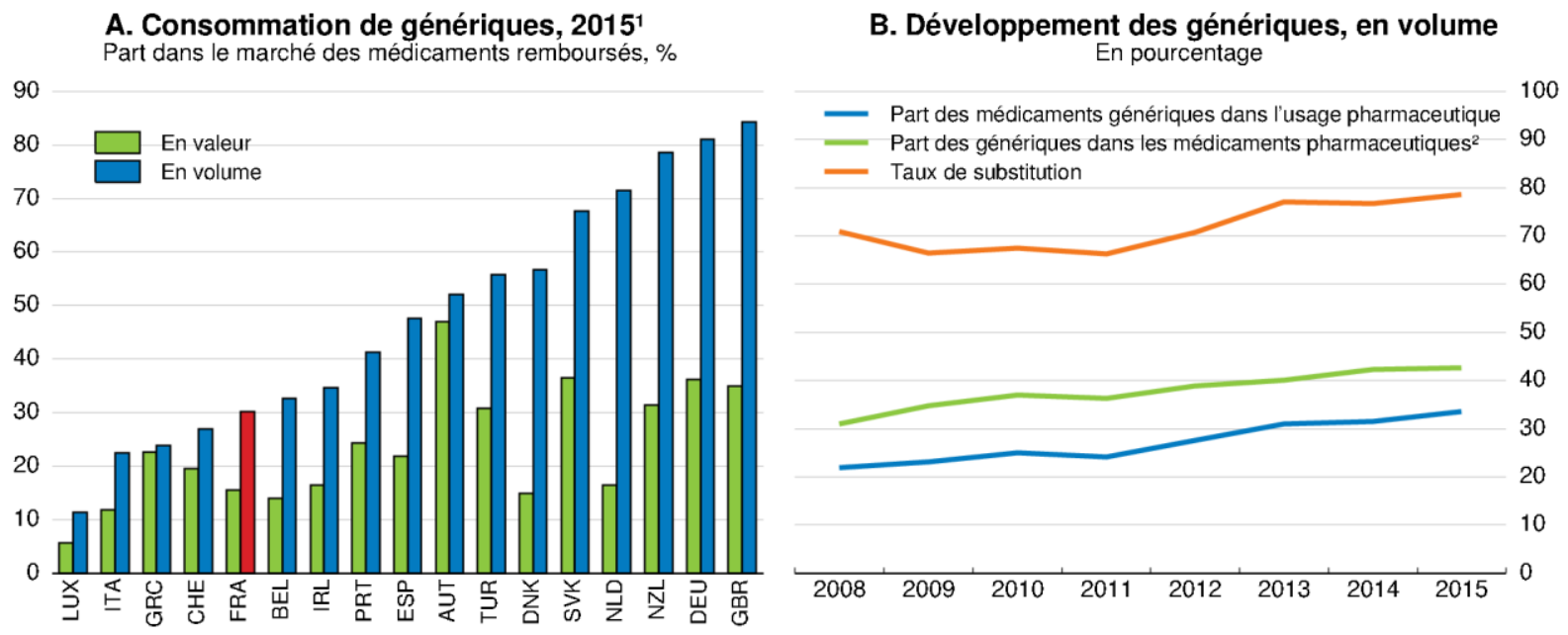

1. Ou dernière année disponible. Part dans le marché des médicaments remboursés.

2. Part des produits pharmaceutiques vendus associés à un générique de substitution.

Source: OCDE (2017), Base de données des Statistiques de l'OCDE sur la santé ; Comité économique des produits de santé (2015 et 2016), Rapport d'activité du CEPS 2014-2015 et Rapport d'activité du CEPS 2015.

Comme dans de nombreux pays de l'OCDE, les autorités françaises ont tendance à réglementer le prix des génériques par rapport au prix du princeps et à des taux d'escompte fixes. Des appels d'offres pour de nouveaux génériques pourraient permettre de faire baisser davantage les prix. Les fabricants de génériques feraient une offre sur le prix d'un produit pharmaceutique donné, et les pharmacies n'auraient pas d'autre choix que de stocker le produit du fabricant ayant remporté le marché. Ces processus concurrentiels sont utilisés en Nouvelle-Zélande, aux Pays-Bas et en Allemagne avec un certain succès.

Accroître la part des génériques nécessiterait de nouvelles réformes dans les soins primaires. Les pharmaciens sont les principaux promoteurs des médicaments génériques depuis 1999 (Autorité de la 
concurrence, 2013). Ils sont libres de proposer des génériques de substitution figurant sur la liste autorisée, les réglementations leur garantissent les mêmes marges (en valeur absolue) pour les génériques et les produits de référence, malgré des prix de détail inférieurs. En outre, ils peuvent facturer des coûts de revente importants aux grossistes, qui ne peuvent excéder $40 \%$ du prix de vente des génériques contre $2.5 \%$ pour les autres produits pharmaceutiques. Cela crée des risques de collusion entre les pharmaciens et les grossistes et des accords de fixation des prix (DGCCRF, 2016). La CNAMTS a également versé des primes aux pharmaciens pour avoir augmenté le taux de substitution de certaines molécules depuis 2012 sous forme de rémunération sur objectifs de santé publique. Par conséquent, les mécanismes d'incitation à l'utilisation des génériques sont coûteux (Cour des comptes, 2014c).

Réformer la rémunération des pharmaciens serait bénéfique pour le développement des génériques et pour encourager l'amélioration de la qualité de leur service. Rééquilibrer une partie du soutien dont bénéficient les génériques au profit de la rémunération des pharmaciens pour les services de soins tels que le conseil, la coordination entre les professionnels de santé et le suivi des patients réduirait la dépendance de leurs revenus aux ventes de produits pharmaceutiques et valoriserait leur fonction de conseillers. En parallèle, leurs missions de santé publique pourraient être renforcées, notamment en ce qui concerne le suivi du traitement des patients atteints de maladies chroniques, comme le prévoit une loi de 2009 (Loi hôpital, patients, santé, territoires) qui n’est pas encore pleinement appliquée.

Il serait également utile de renforcer les incitations des médecins généralistes à prescrire des médicaments génériques. La CNAMTS a mis en place de telles incitations par le biais de son système de rémunération aux résultats lancé en 2009, et les a quelque peu renforcées en 2012. Toutefois, il faudrait mieux développer les informations sur les génériques et les autres solutions moins coûteuses, notamment en ciblant les prestataires lors de la promotion prévue des recommandations de soins (ministère des Affaires sociales, de la Santé et des Droits des femmes, 2015). En outre, le développement des prescriptions en dénomination commune internationale (DCI), rares en 2013 (Cour des comptes, 2014c) mais rendues obligatoires en 2015, devrait également faciliter l'utilisation des génériques (Belloni et al., 2016).

Une réforme visant à inciter davantage les patients à la consommation de médicaments génériques et des biosimilaires pourrait réduire les dépenses pharmaceutiques. Depuis 2003, le tarif forfaitaire de responsabilité (TFR) s'applique à tous les produits pharmaceutiques appartenant à un même groupe générique dont le taux de substitution est inférieur à $80 \%$ après 30 ans de mise sur le marché des médicaments génériques: les patients doivent payer la différence entre le TFR (proche du prix du générique le moins cher) et le prix des non- génériques ou des autres génériques plus coûteux. Dans les faits, les laboratoires alignent leur prix sur le TFR rendant le surcoût pour les patients nul (Caby et Zafar, 2017). En outre, depuis 2010, les patients doivent payer en avance leurs médicaments et se faire rembourser plus tard lorsqu'ils refusent un générique. Il s'agit d'un pas dans la bonne direction. Toutefois, le TFR n'était appliqué qu'à $16 \%$ des génériques en 2015 (Drees, 2016c). Il pourrait être appliqué beaucoup plus largement.

Plus généralement, un renforcement des informations et des incitations visant les professionnels de santé permettrait d'abaisser les prescriptions potentiellement inappropriées et le recours excessif aux antibiotiques. En France, le taux de prescription d'antibiotiques est l'une des cibles de la rémunération sur objectifs de santé publique des médecins, qui peuvent se situer par rapport aux moyennes régionales (CNAMTS, 2016b). En outre, la stratégie 2016 relative aux antibiotiques afin de diminuer la résistance aux antibiotiques prévoit des campagnes d'information supplémentaires, une formation des professionnels de santé et une hausse des dépenses au titre de la recherche et de la surveillance (CIS, 2016). Toutefois, ce retour d'informations aux médecins et les campagnes d'informations pourraient être mieux ciblés, puisqu'ils visent en termes généraux les professionnels de santé et les patients. En effet, au Royaume-Uni, les retours d'informations qui visaient les médecins prescrivant le plus 
d'antibiotiques ont permis de réduire efficacement le recours aux antibiotiques, alors que les campagnes d'informations visant les patients ont eu des effets limités (OCDE, 2017b).

Au-delà des soins primaires, des efforts considérables ont été faits pour améliorer la consommation de produits pharmaceutiques dans les hôpitaux. Les ventes aux hôpitaux représentent environ un quart de l'ensemble des ventes de produits pharmaceutiques en France, mais les génériques ne représentaient que $2.3 \%$, en valeur, des ventes aux hôpitaux en 2013 (ANSM, 2014). Cette faible part s'explique en partie par les différentes pathologies des patients et des traitements plus lourds et plus innovants dans les hôpitaux (OCDE, 2015b), mais les entreprises pharmaceutiques ont également souvent tendance à leur vendre des médicaments de marque à prix réduits pour gagner un marché plus large (Dahan, 2016). En effet, les prescriptions hospitalières ont des répercussions importantes puisque les patients ont tendance à suivre les mêmes traitements après leur sortie (Cubaynes et al., 2011). Les projets des autorités visant à améliorer la coordination des prescriptions entre les hôpitaux et les soins de ville et à mieux intégrer les retombées des décisions d'achats hospitaliers sur les soins de ville sont bienvenus (ministère des Affaires sociales, de la Santé et des Droits des femmes, 2015).

Il serait également utile de renforcer l'indépendance des professionnels de santé vis-à-vis de l'industrie pharmaceutique. Les déclarations publiques d'absence de conflits d'intérêts sont insuffisamment contrôlées et mises à jour. De plus, le financement des activités de recherche, de la formation continue et des autres relations par les laboratoires devrait être clairement identifié (Cour des comptes, 2016e). Par exemple, le groupement des hôpitaux parisiens (AP-HP) a créé une agence indépendante pour financer ses activités de recherche conjointes avec le secteur pharmaceutique, et cette initiative pourrait être généralisée. L'essor actuel des achats conjoints des hôpitaux publics devrait limiter les risques de conflits d'intérêts pour les médecins hospitaliers et les autres prestataires et organismes, mais il faudrait mieux tenir compte de l'expertise en matière de produits médicaux et d'achats publics dans les carrières et la rémunération des chercheurs en médecine.

\section{Investir dans la prévention et adopter des styles de vie plus sains}

Les hommes français affichent un taux particulièrement élevé de décès prématurés par accident, ou dus à des habitudes néfastes pour la santé, comme le tabagisme et la consommation d'alcool, qui sont les causes les plus fréquentes de mortalité évitable. La lutte contre la consommation excessive de tabac et d'alcool pourrait également améliorer les résultats sur le plan de la santé, permettrait de réaliser d'importantes économies à long terme et contribuerait à réduire les disparités sociales. En effet, la consommation d'alcool et de tabac et l'obésité sont plus fréquentes chez les ménages les plus modestes. Néanmoins, ces publics étant plus difficiles à toucher par les campagnes de prévention traditionnelles, il conviendrait de développer des nouvelles méthodes ciblées à leur destination. Toutefois, la lutte contre le chômage de longue durée et les problèmes de mobilité sociale sont également des déterminants essentiels des résultats sanitaires à plus long terme (Persson et Rossin-Slater, 2016). Dans le même temps, il serait très avantageux d'améliorer les résultats environnementaux et de réduire les risques professionnels.

\section{Améliorer la prévention et lutter contre les comportements à risque}

La prévention des conduites addictives (tabac-alcool) et la prévention de l'obésité des jeunes enfants figurent parmi les engagements des partenaires conventionnels. Le pourcentage de la population qui fume quotidiennement en France demeure très supérieur à la moyenne de l'OCDE, notamment pour les femmes (graphique 20, partie A). Le tabagisme quotidien demeure aussi particulièrement élevé chez les jeunes (Spilka et al., 2015), alors que le tabagisme est à l'origine de quelque 78000 décès par an (Ribassin-Majed et Hill, 2015). Les autorités ont élaboré plusieurs stratégies pour faire face à ce problème, en particulier en augmentant régulièrement les taxes sur le tabac (partie B); le nouveau 
gouvernement entend poursuivre cette hausse en portant progressivement le prix du paquet de cigarettes à 10 euros. Par ailleurs, un programme national lancé en 2014 ainsi que la loi de modernisation de notre système de santé de 2016 ont intensifié les campagnes d'information et de prévention, notamment pour les jeunes, et ont mis en place des interdictions publiques de fumer ainsi que le paquet de tabac neutre. Toutefois, ces mesures pourraient être utilement complétées par l'inclusion du suivi des patients dépendants au tabac dans la rémunération des médecins généralistes, et l'élaboration de stratégies d'e-coaching (CNAMTS, 2016a).

\section{Graphique 20. Consommation de tabac}

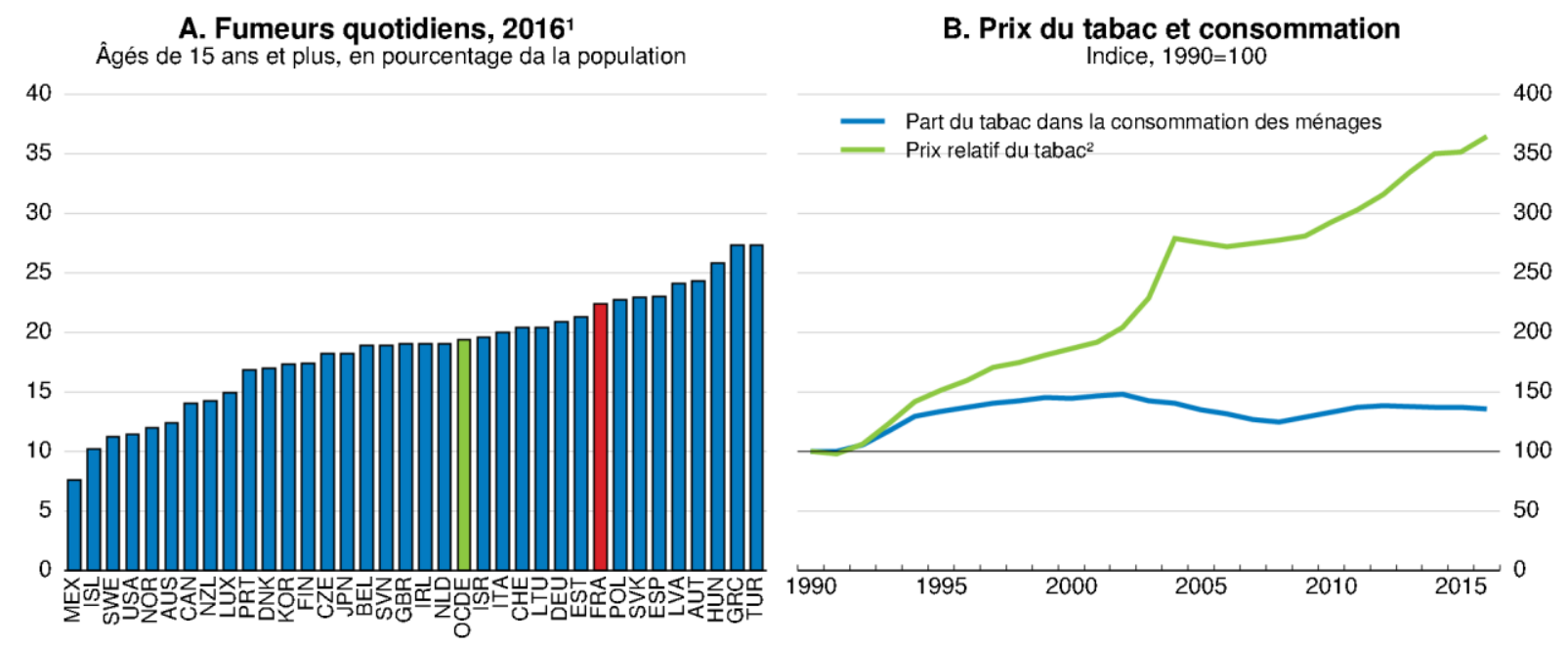

1. Ou dernière année disponible.

2. Évolution par rapport à l'indice des prix à la consommation (IPC).

Source: OCDE (2017), Base de données des Statistiques de l'OCDE sur la santé ; INSEE (2017), Banque de données macroéconomiques (BDM).

La consommation d'alcool a diminué en France au cours des 30 dernières années, mais demeure parmi les plus importantes de l'OCDE (graphique 21, partie A). Elle est à l'origine de quelque 49000 décès par an en France (Guérin et al., 2013) et d'un grand nombre d'hospitalisations évitables (Paille et Reynaud, 2015). En outre, les buveurs font payer un lourd tribut à leurs concitoyens en termes de coûts externes, comme les accidents de la route et la violence domestique. Le gouvernement a adopté un large éventail de mesures pour restreindre la publicité pour les boissons alcooliques et les ventes d'alcool sur place ou à emporter (p. ex. aux mineurs et dans les stations-service). Toutefois, par rapport à d'autres pays de l'OCDE, la France continue de taxer faiblement l'alcool, notamment le vin (OCDE, 2015c). Il convient également de noter que cette taxation est indépendante du degré d'alcool depuis 1990 (partie B). Au-delà d'une taxation plus élevée, des interdictions plus efficaces de la publicité pour l'alcool pourraient faire baisser la consommation totale, avec un impact vraisemblablement plus important sur les gros buveurs (OCDE, 2015c). L'interdiction de la publicité dans les médias numériques, le renforcement de la prévention et du suivi des populations vulnérables, tout comme le durcissement des sanctions contre les ventes illégales et le développement de technologies empêchant la conduite en état d'ivresse, abaisseraient considérablement le coût social de la consommation d'alcool (Cour des comptes, 2016f). 


\section{Graphique 21. Consommation d'alcool et prix}
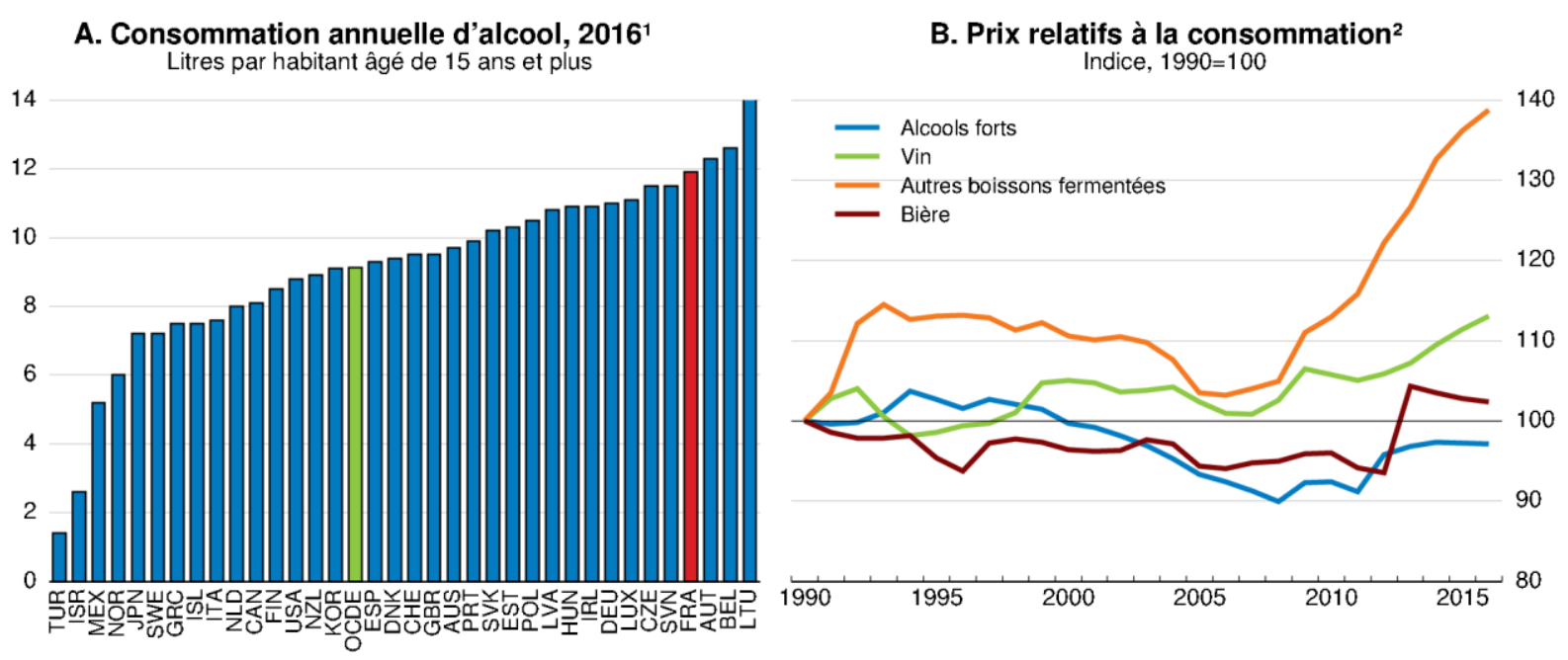

1. Ou dernière année disponible.

2. Évolution par rapport à l'indice global des prix à la consommation (IPC).

Source: OCDE (2017), Base de données des Statistiques de l'OCDE sur la santé ; INSEE (2017), Banque de données macroéconomiques (BDM).

Les coûts sociaux de l'excès de poids sont également importants. Les taux de surpoids et d'obésité augmentent rapidement en France (OCDE, 2014b), bien qu'ils demeurent parmi les plus faibles de l'OCDE (graphique 22, partie A). Les coûts sociaux annuels de l'excès de poids sont à peu près comparables aux coûts du tabagisme et de la consommation d'alcool (Caby, 2016). La consommation de sucre a augmenté au cours des 20 dernières années (parties $\mathbf{B}$ à $\mathbf{D}$ ) car la consommation des ménages s'est déplacée vers des produits sucrés et des aliments prêts à l'emploi, bien que la consommation de matières grasses ait été globalement stable à un niveau élevé. La hausse du prix des produits sucrés à l'aide de judicieux leviers fiscaux pourrait promouvoir des régimes alimentaires plus sains, puisque la consommation de ces produits sucrés paraît avoir eu une élasticité prix élevée au cours des 50 dernières années en France (Larochette et Sanchez-Gonzalez, 2015 ; Sassi et al., 2013). La taxe française sur les boissons sucrées et édulcorées mise en place en 2012 pourrait être ajustée et étendue à d'autres produits. En effet, elle est fondée sur la valeur de certains produits plutôt que sur leur teneur en sucres, ce qui peut limiter ses répercussions sur les consommateurs et son efficacité (Bonnet et Réquillart, 2013 ; Berardi et al., 2016). L'intégration de cette approche au Programme national nutrition santé (PNNS) et à l'étiquetage facultatif des produits alimentaires en 2017, fondé sur des critères de santé, permettraient de sensibiliser les consommateurs. Par ailleurs, une expérimentation de prise en charge des enfants de 3 à 8 ans à risque d'obésité sera conduite en 2017-18.

\section{Réduire les risques professionnels}

Par rapport à d'autres pays européens, les déclarations d'accidents du travail sont relativement fréquentes, même après prise en compte des différences de structure des secteurs (graphique 23, parties A et B). Cependant, les accidents du travail ont régulièrement reculé (partie $\mathbf{C}$ ), bien qu'à un rythme plus lent que dans d'autres pays européens (Premier Ministre, 2011). En même temps, les données officielles montrent que la prévalence des troubles musculosquelettiques et des autres maladies professionnelles a rapidement augmenté, même s'ils restent sous-déclarés (Drees, 2015). Les progrès sont hétérogènes d'une entreprise à l'autre et la fréquence des accidents demeure élevée dans de nombreuses PME (partie D). 


\section{Graphique 22. Prévalence de l'obésité et régime alimentaire}

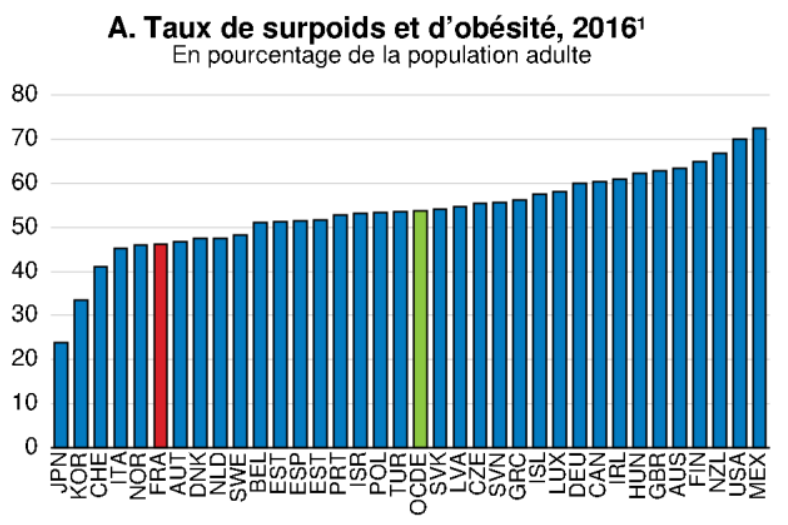

C. Consommation de sucre

En kilos par habitant, par an

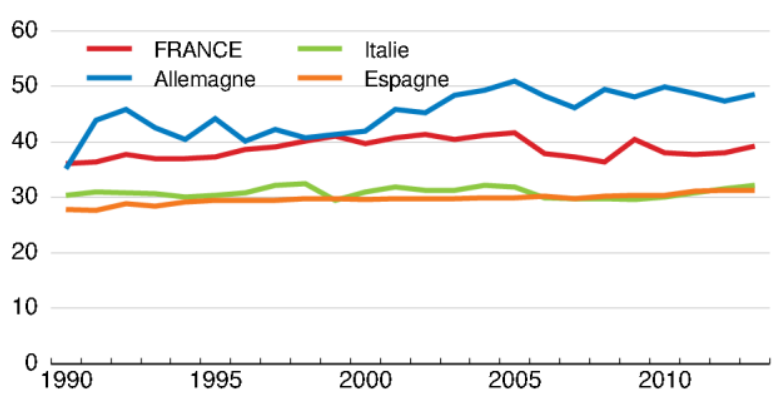

B. Consommation de matières grasses Grammes par habitant par jour

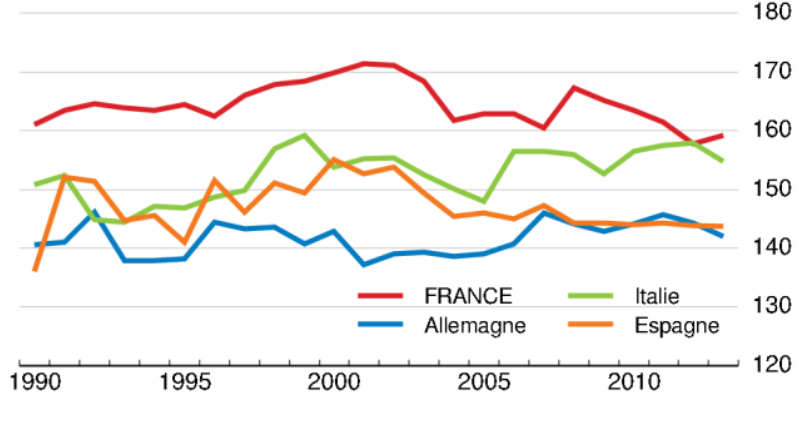

D. Composition de la consommation alimentaire des ménages En pourcentage

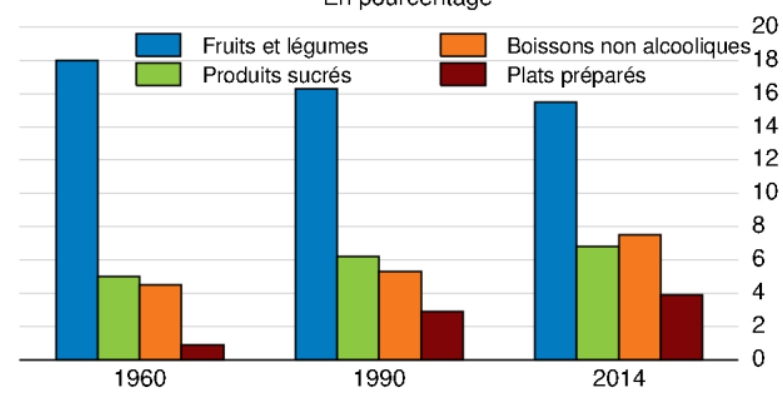

1. Ou dernière année disponible. Les taux de surpoids et d'obésité sont auto-déclarés dans certains pays.

Source : OCDE (2017), Base de données des Statistiques de l'OCDE sur la santé ; Larochette, B. et J. Sanchez-Gonzalez (2015), "Cinquante ans de consommation alimentaire: une croissance modérée, mais de profonds changements ", Insee Première, $n^{\circ} 1568$.

L'absentéisme dû aux accidents du travail et maladies professionnelles augmente. Les jours d'absence par travailleur pour cause d'accidents du travail et maladies professionnelles ont augmenté de $25 \%$ au cours des 15 dernières années (graphique 24, partie A), et les indemnités pour risques professionnels ont augmenté de $15 \%$ relativement au PIB depuis 2007 (partie B). La sous-déclaration des maladies professionnelles et des accidents (Sécurité sociale, 2014) réduit probablement les incitations à la prévention pour les entreprises. La CNAMTS gère le principal dispositif de prise en charge des risques professionnels (AT-MP), en faisant reposer en partie les cotisations des entreprises sur leurs expériences. Avec les autres assurances obligatoires, elle couvre également les indemnités journalières pour raisons de santé (IJ). L'absentéisme pour des blessures qui ne sont pas clairement liées à un accident du travail peut être pris en charge par l'un ou l'autre dispositif : environ $20 \%$ des demandes dues à des accidents du travail et des maladies professionnelles seraient orientées vers les dispositifs d'assurance maladie obligatoires (Askenazy, 2006; Cour des comptes, 2012). En effet, la partie de l'évaluation de l'expérience des entreprises du programme AT-MP peut également avoir des effets indésirables, en décourageant le signalement d'accidents et de problèmes de santé et en réduisant l'employabilité de populations vulnérables, notamment les personnes handicapées et les travailleurs âgés.

Une amélioration du ciblage et du suivi des programmes de prévention pourrait réduire les risques professionnels. C'est l'ambition du troisième plan santé au travail 2016-20 qui place la prévention primaire au centre des préoccupations. La relation entre les accidents du travail et la taille de l'entreprise suit une courbe en cloche avec une phase descendante commençant autour de 50 salariés, même après prise en compte de la gravité des accidents (Garoche, 2016). Toutefois, certaines subventions à la prévention des 
risques sont accessibles uniquement aux PME de moins de 50 salariés (Cour des comptes, 2013), et les comités d'hygiène, de sécurité et des conditions de travail (CHSCT) font souvent défaut dans les entreprises de plus de 50 salariés, bien qu'ils soient obligatoires (Breda, 2016). Les petites entreprises et les administrations locales mettent en ouvre peu de mesures de prévention. En 2013, seuls $60 \%$ des employeurs avaient pris des mesures au cours des 12 mois précédents, et moins de la moitié avait élaboré ou mis à jour un document unique d'évaluation des risques professionnels, pourtant obligatoire (Amira, 2016). Il est bienvenu d'axer la prévention sur les PME, comme prévu dans le Plan santé au travail 2016-2020. En effet, la mise en place des CHSCT dans les PME favorise une meilleure connaissance des risques pour la santé (Coutrot, 2009). La loi travail de 2016 pourrait également contribuer à améliorer la prévention. Elle a dégagé du temps pour l'action de prévention en entreprise, en créant une visite d'information et de prévention et en permettant la montée en charge d'équipes pluridisciplinaires.

\section{Graphique 23. Risques professionnels}

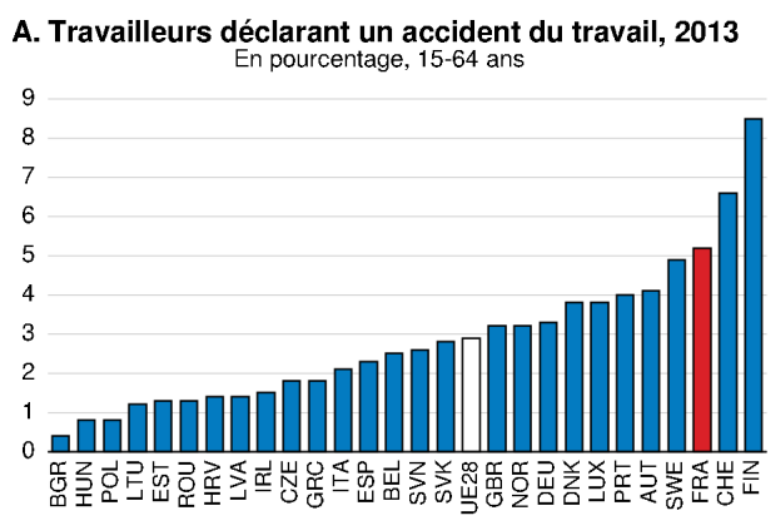

C. Accidents du travail ${ }^{2}$ et maladies professionnelles ${ }^{3}$ Pour 1000 salariés

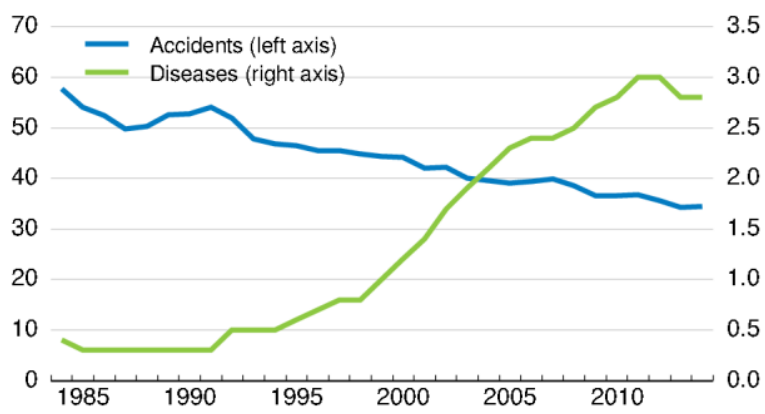

\section{B. Taux standardisé d'accidents du travail non mortels' Pour 1000 salariés}

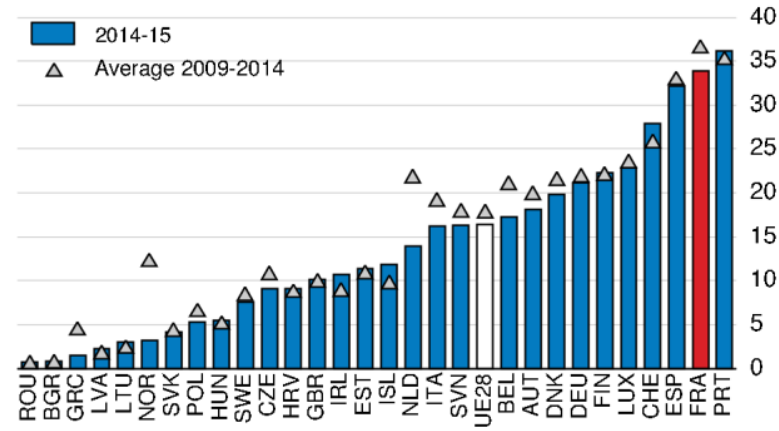

D. Taille de l'entreprise et fréquence des accidents Pour 1000 salariés

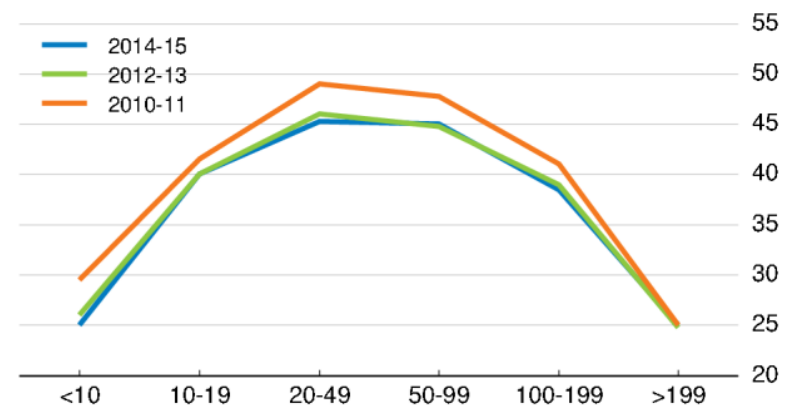

1. Les déclarations d'accidents professionnels sont corrigées en tenant compte de la structure des secteurs. Le taux d'incidence standardisé s'appuie sur l'hypothèse que les secteurs économiques de chaque pays ont la même taille relative en termes de population de référence que les secteurs au niveau de l'UE.

2. Les accidents professionnels sont des accidents entraînant une journée d'absence au travail.

3. Les données concernent uniquement les salariés affiliés au régime professionnel obligatoire en France métropolitaine entre 1984 et 1999, et tous les travailleurs les années suivantes.

Source: Eurostat (2017), Accidents du travail et problèmes de santé liés au travail; Assurance-Maladie Risques Professionnels (2016), Bases de données: principaux indicateurs AT, 2010 à 2015, www.risquesprofessionnels.ameli.fr/. 
Graphique 24. Congés maladie et jours d'absence liés aux accidents du travail et maladies professionnelles
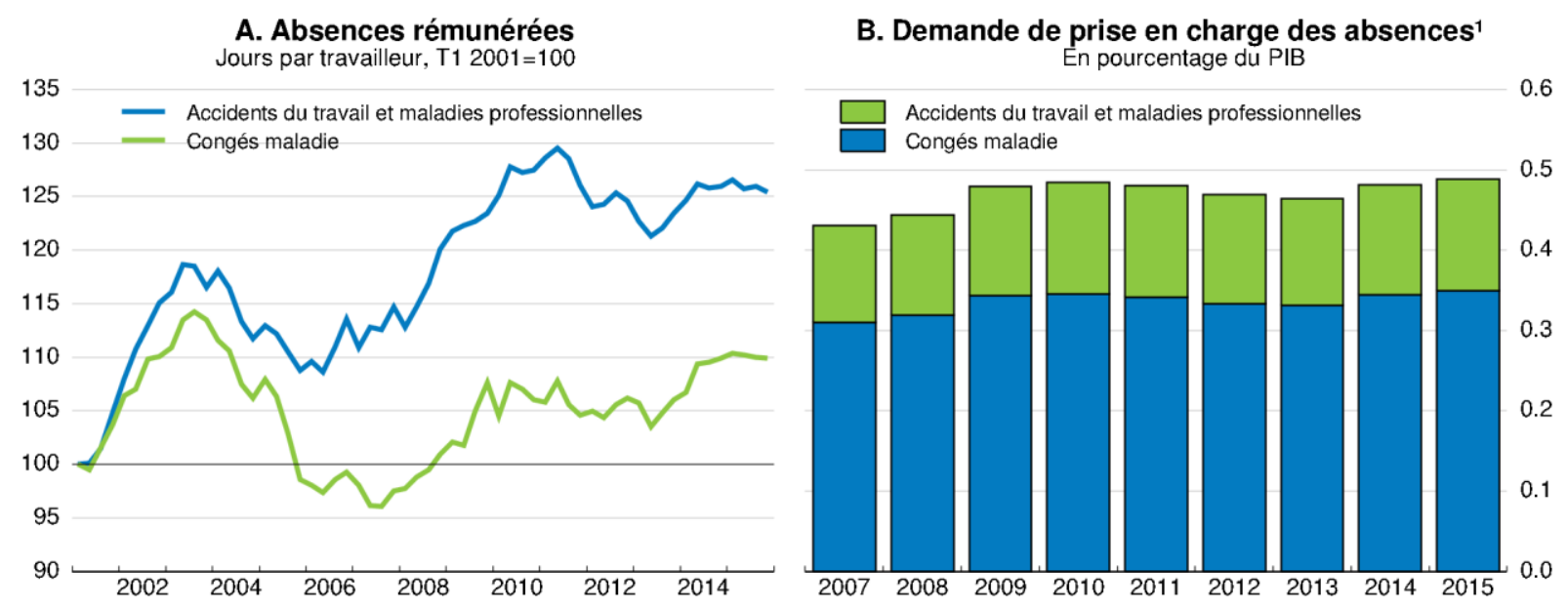

1. Prise en charge par la CNAMTS, à l'exclusion des coûts pris en charge par les autres régimes obligatoires, notamment des fonctionnaires, des travailleurs agricoles et des travailleurs indépendants, et par les organismes complémentaires.

Source : Drees (2016), Les dépenses de santé en 2015 - Résultats des comptes de la santé.

La réduction des risques professionnels et de l'absentéisme dans le secteur public permettra également de réaliser quelques économies. Les régimes publics prenant en charge les risques professionnels dans les hôpitaux publics et les administrations centrales et locales incitent peu à la prévention. Au minimum, la convergence des règles vers le dispositif AT-MP aurait des effets bénéfiques sur l'efficience et l'équité, puisque ce dispositif couvre déjà les non-fonctionnaires qui travaillent pour les établissements de santé et les collectivités territoriales. Dans le même temps, la gestion des ressources humaines pourrait être renforcée dans les administrations locales, dans lesquelles l'absentéisme est important et de nombreux contrats prévoient un temps de travail obligatoire inférieur à la semaine légale de travail, ce qui augmente la masse salariale (Cour des comptes, $2016 \mathrm{~g}$ ). L'amélioration des pratiques de gestion et la réduction des sureffectifs et des doublons dans les administrations locales pourraient avoir un effet positif. Toutefois, les employés du secteur public déclarant avoir moins d'autonomie ou des relations conflictuelles au travail sont plus souvent absents, même après prise en compte de l'âge, de la profession et du niveau d'instruction (Davie, 2015).

\section{Garantir des conditions environnementales saines}

La France obtient de bons résultats en termes de qualité globale de l'environnement, bien que les pics de pollution dans certaines villes aient des effets négatifs sur la santé. La pollution aux particules fines (PM10) est très inférieure à la moyenne de l'OCDE et recule régulièrement (graphique 25, partie A ; OCDE, 2016e), bien qu'elle soit plus importante dans certaines villes. Les concentrations d'ozone au niveau du sol ont augmenté et dépassent régulièrement les seuils recommandés dans certaines villes en période de forte chaleur (partie B), ce qui pourrait accroître l'incidence des maladies respiratoires. Des estimations récentes suggèrent que les pics de pollution atmosphérique pourraient être à l'origine de quelque 48000 décès par an en France ( $9 \%$ du total des décès), et réduire l'espérance de vie à l'âge de 30 ans de plus de deux ans (Santé publique France, 2016). Les transports routiers sont un contributeur majeur de cette évolution : il serait approprié d'accroître la charge fiscale pesant sur le carburant diesel, une source majeure de pollution aux PM10, comme cela a été prévu. 


\section{Graphique 25. Pollution atmosphérique urbaine}

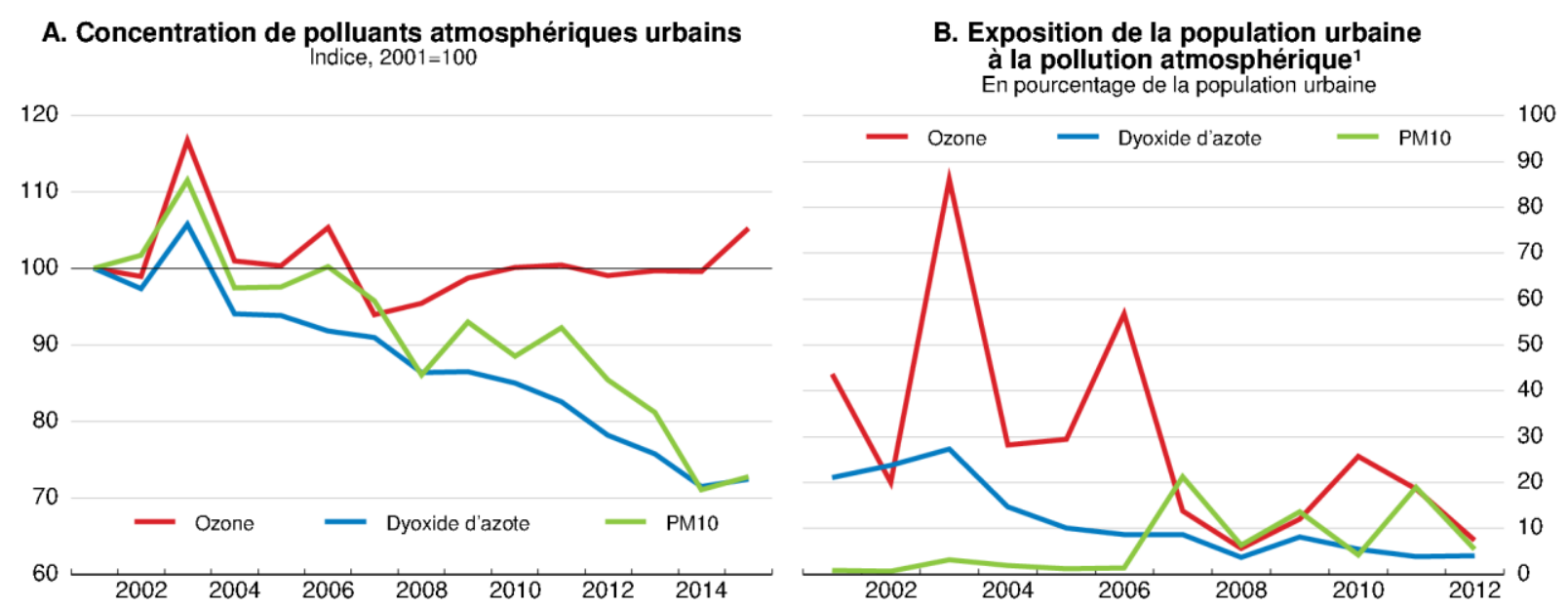

1. La pollution atmosphérique est définie comme la concentration d'une substance chimique donnée au-dessus des seuils réglementaires européens (concentrations quotidiennes de PM10 supérieures à $50 \mu \mathrm{g} / \mathrm{m} 3$ plus de 35 jours par an, concentrations moyennes quotidiennes sur 8 heures d' $\mathrm{O}_{3}$ supérieures à $120 \mu \mathrm{g} / \mathrm{m}^{3}$ plus de 25 jours par an, et concentrations annuelles moyennes de $\mathrm{NO}_{2}$ supérieures à $40 \mu \mathrm{g} / \mathrm{m}^{3}$ ).

Source : Commissariat général au Développement durable (2017), Chiffres clés de l'environnement, Édition 2016 ; European Energy Agency (2014), Percentage of urban population resident in areas where pollutant concentrations are higher than selected limit/target values, 2000-2012 (EU-28).

Une meilleure intégration des politiques locales et nationales sur la qualité de l'air contribuerait à réduire la pollution atmosphérique locale. La Commission européenne (2013b et 2017) a engagé des poursuites contre la France du fait qu'elle aurait échoué à réduire les concentrations locales de PM10 et de dioxyde d'azote. Les administrations régionales, les grandes villes et les zones métropolitaines doivent élaborer des plans de qualité de l'air depuis 1996. Bien que ces plans autorisent l'administration centrale à limiter les nouvelles installations d'activités polluantes, certains sont principalement indicatifs (OCDE, 2016e). La préparation de documents stratégiques infranationaux n'est pas complètement coordonnée, et il y a peu d'évaluations des mesures locales et de leur impact budgétaire (Cour des comptes, 2016h). Le précédent gouvernement a proposé un projet de stratégie pour la période 2016-20, qui prévoit notamment une hausse des taxes sur le diesel, un soutien et un contrôle complémentaires des administrations locales, des rapports de progression annuels et d'autres évaluations de l'impact. Ces mesures devraient toutes être rapidement mises en œuvre. En même temps, la taxation du diesel et des véhicules fortement polluants qui bénéficient de subventions et de réductions d'impôt, tels que les taxis, les véhicules de transport en commun et certains véhicules lourds, devrait augmenter davantage en fonction de leurs dommages environnementaux et sanitaires.

La France est également l'un des principaux consommateurs de pesticides du monde, ce qui pose des risques considérables pour la santé, l'environnement, la biodiversité et les écosystèmes. La consommation de pesticides a augmenté de $29 \%$ en France entre 2008 et 2014 (OCDE, 2016e), et de nombreux agriculteurs souffrent d'affections de longue durée liées aux pesticides. Bien que des études complémentaires soient nécessaires pour distinguer les effets des différents polluants (ANSES, 2016; Cour des comptes, 2016h), les pouvoirs publics doivent encourager la transition vers une agriculture plus verte. La suppression en 2014 du taux de TVA réduit sur les fertilisants et les pesticides est bienvenue, et il serait utile d'intégrer les effets sur la qualité de l'air et de l'eau aux procédures d'autorisation des nouveaux fertilisants (Husson et Aïchi, 2015). Ces effets devraient également être reflétés dans la fiscalité en vigueur pour les produits phytosanitaires et la redevance pour pollution diffuse. Signe encourageant, l'agriculture biologique a progressé de plus de $60 \%$ au cours des cinq dernières années (Eurostat, 2016). 


\section{Recommandations pour améliorer l'efficience du système de santé}

\section{Améliorer le financement des dépenses de santé}

- Évaluer plus fréquemment l'efficacité des produits pharmaceutiques et des traitements, et les tarifs associés. Renforcer comme prévu le lien entre les résultats des évaluations et les taux de remboursement. Promouvoir le suivi et l'analyse de la qualité des soins de santé.

- Supprimer les allègements fiscaux des particuliers et entreprises pour les assurances complémentaires collectives, en tenant compte de leur impact sur le coût du travail. À la place, faciliter l'accès aux soins de santé pour les ménages à bas revenu en s'appuyant sur les données administratives pour déterminer s'ils peuvent accéder à différents programmes d'aides à la complémentaire santé. Continuer la convergence des différents régimes obligatoires afin de passer à un régime unique.

- $\quad$ Reconsidérer le partage des biens et services couverts par l'assurance obligatoire et les régimes d'assurance complémentaires en s'assurant que le champ des soins couverts par les complémentaires ne soit pas trop ciblé afin d'éviter les phénomènes d'anti-sélection.

\section{Promouvoir des soins appropriés et la maîtrise des coûts}

- Augmenter la rémunération des praticiens de santé pour la prévention et le traitement des maladies chroniques complexes, ce qui permettrait de réduire le nombre de soins, des ordonnances et la vente de médicaments.

- Renforcer la coordination entre les professionnels de la santé en développant des dossiers médicaux électroniques.

- Faciliter la contractualisation des relations entre les régimes d'assurance et les groupements de prestataires afin de mieux desservir les zones en sous-densité.

- Améliorer les possibilités de formation commune initiale et continue pour les professionnels de santé, en développant en particulier un socle de formation commune complémentaire de manière à renforcer la coordination entre professionnels de santé. Renforcer le rôle des infirmiers et des pharmaciens en tant que praticiens. Envisager une réforme plus vaste des professions de santé réglementées en définissant leurs activités en termes d'objectifs généraux.

- $\quad$ Ajuster le système de tarification à l'activité dans les hôpitaux (GHS) afin de mieux rémunérer l'efficience et la qualité dans les hôpitaux publics et privés.

- Renforcer l'autonomie des gestionnaires des hôpitaux publics en augmentant leur indépendance vis-à-vis des administrations locales et en leur permettant d'appliquer leurs propres politiques de ressources humaines, sous réserve d'un cadrage régional.

- Réduire les dépenses pharmaceutiques en augmentant l'usage des génériques, en concevant des informations ciblées pour les hôpitaux et les prestataires de soins primaires afin d'améliorer la pertinence des prescriptions, et en appliquant des plafonds de remboursements à un plus large éventail de médicaments.

Investir dans la prévention, la promotion de modes de vie plus sains et la protection de l'environnement

- Accroître les droits d'accises sur l'alcool et les campagnes d'information visant les consommateurs d'alcool. Réformer les droits d'accises sur les boissons sucrées en prenant en compte leur teneur en sucre, et les étendre à d'autres produits alimentaires.

- Poursuivre le renforcement de la fiscalité sur les carburants diesel et les véhicules fortement polluants. Intégrer l'évaluation de l'impact de la qualité de l'air et de l'eau aux procédures d'autorisation des produits agricoles. 


\section{REFERENCES}

Albouy, V., E. Bretin, N. Carnot and M. Deprez (2009), « Les dépenses de santé en France : déterminants et impact du vieillissement à l'horizon 2050 », Documents de travail de la DGTPE, n 2009-11.

Albouy, V. et M. Deprez (2008), «Mode de rémunération des médecins », Trésor-Éco, n ${ }^{\circ}$ 2, Direction générale du Trésor.

Amira, S. (2016), « La prévention des risques professionnels », DARES Résultats, $\mathrm{n}^{\circ} 013$.

Anguis, M. (2017), «En 2016, 7700 étudiants affectés à l'issue des premières épreuves classantes nationales informatisées », Études et résultats, $\mathrm{n}^{\circ} 1006$, Drees.

ANSES (2016), Expositions professionnelles aux pesticides en agriculture - Volume $n^{\circ} 1$ : Volume central, Agence nationale de sécurité sanitaire de l'alimentation, de l'environnement et du travail.

ANSM (2014), Analyse des ventes de médicaments en France en 2013, Agence nationale de sécurité du médicament et des produits de santé.

Askenazy, P. (2006), «Les aspects économiques de la prévention et de la réparation des risques professionnels », Actualité et dossier en santé publique, $\mathrm{n}^{\circ} 2006$, pp. 39-41.

Askenazy, P., B. Dormont, P.-Y. Geoffard et V.Paris (2013), « Pour un système de santé plus efficace », Les notes $d u$ conseil d'analyse économique, $\mathrm{n}^{\circ} 8$.

ATIH (2016), État des lieux 2014 sur l'activité de chirurgie ambulatoire (nouveau périmètre), Agence technique de l'information sur l'hospitalisation.

Autorité de la concurrence (2013), Avis $n^{\circ}$ 13-A-24 du 19 décembre 2013 relatif au fonctionnement de la concurrence dans le secteur de la distribution du médicament à usage humain en ville, Paris.

Autorité de la concurrence (2016a), Avis $n^{\circ} 16-A-11$ du 11 mai 2016 relatif à un projet de décret portant code de déontologie des infirmiers, Paris.

Autorité de la concurrence (2016b), Avis $n^{\circ} 16-A-09$ du 26 avril 2016 relatif à deux projets d'arrêtés concernant le commerce électronique de médicaments, Paris.

Auvigné, F., X. Chastel., B. Ravignon, V. Caire, A. Laurenty et C. Gardette (2013), « Les coûts de gestion de l'assurance maladie », Rapport de l'Inspection générale des finances et de l'Inspection générale des affaires sociales, IGF-IGAS, Paris.

Bachelet, M. et M. Anguis (2017), «Les médecins d'ici à 2040 : une population plus jeune, plus féminisée et plus souvent salariée », Études et Résultats, n 1011, Drees, Mai.

Bartel, A., N. Beaulieu, C. Phibbs et P. Stone (2014), «Human Capital and Productivity in a Team Environment: Evidence from the Healthcare Sector», American Economic Journal: Applied Economics, vol. 6, n 2, pp. 231-59. 
Baudis, D. (2014), «Les refus de soins opposés aux bénéficiaires de la CMU-C, de l'ACS et de l'AME », Rapport remis au Premier ministre.

Baulinet, C., M. Duraffourg et S. Vanackere (2012), «La fiscalité spécifique applicable aux produits de santé et à l'industrie qui les fabrique », Rapport de l'IGAS et de l'IGF, Paris.

Belloni, A., D. Morgan et V. Paris (2016), « Pharmaceutical Expenditure and Policies: Past Trends And Future Challenges », OECD Health Working Papers, n 87, Éditions OCDE.

Berardi, M., P. Sevestre, M. Tepaut et A. Vigneron (2016), «The impact of a "soda tax" on prices: Evidence from French micro data », Applied Economics, vol. 48 n 41, pp. 3976-94.

Berchet, C. (2015), «Emergency Care Services: Trends, Drivers and Interventions to Manage the Demand », OECD Health Working Papers, n ${ }^{\circ}$, Éditions OCDE.

Berchet, C. et C. Nader (2016), « The organisation of out-of-hours primary care in OECD countries »,

OECD Health Working Papers, ${ }^{\circ}$ 89, Éditions OCDE.

Bert, T., C. d'Autumne, P. Hausswalt, S. Dupays et M. Weill (2014), « Perspectives de développement de la chirurgie ambulatoire en France », Rapport de l'IGAS et de l'IGF.

Blanpain, N. (2016), «Les hommes cadres vivent toujours 6 ans de plus que les hommes ouvriers », Insee Première, No 1584.

Bloom, N., R. Sadun et J. van Reenen (2014), « Does Management Matter in Healthcare? », Harvard University working paper.

Bonnet, C. et V. Réquillart (2013), « Tax incidence with strategic firms in the soft drink market », Journal of Public Economics, vol. 106, pp. 77-88.

Bozio, A. et B. Dormont (2016), «Gouverner la protection sociale : transparence et efficacité », Note du Conseil d'analyse économique, $\mathrm{n}^{\circ} 28$.

Bozio, A., A. Gramain et C. Martin (2016), «Quelles politiques publiques pour la dépendance ?», Note $d u$ Conseil d'analyse économique, $\mathrm{n}^{\circ} 35$.

Brandt, N. (2017), "Promoting economic opportunities and well-being in poor neighbourhoods", OECD Economics Department Working Papers, No. 1454, OECD Publishing, Paris.

Breda, T. (2016), Les Représentants du Personnel, Presses de Sciences Po, Paris.

Bruant-Bisson, A., P. Castel et M-P. Panel (2012), «Évaluation des effets de la tarification à l'activité sur le management des établissements de santé », Rapport de l'Inspection générale des affaires sociales.

Bruant-Bisson, A. et M. Daudé (2016), «Contribution au rapport au Parlement sur les aides fiscales et sociales à l'acquisition d'une complémentaire santé », Rapport de l'Inspection générale des affaires sociales.

Caby, D. et A. Eidelman (2015), «Quel avenir pour le dispositif de prise en charge des affections de longue durée (ALD) ?», Trésor-Éco, $\mathrm{n}^{\circ} 145$, Direction générale du Trésor. 
Caby, D. (2016), « Obésité : quelles conséquences pour l'économie et comment les limiter ? », Trésor-Éco, $\mathrm{n}^{\circ}$ 179, Direction générale du Trésor.

Caby, D. et J.-D. Zafar (2017), «Quelle politique pour poursuivre la diffusion des médicaments génériques ? », Trésor-Éco, n 199, Direction générale du Trésor.

Chardon, O., N. Guignon et T. de Saint Pol (2015), «La santé des élèves de grande section de maternelle en 2013 : des inégalités sociales dès le plus jeune âge », Études et résultats, $\mathrm{n}^{\circ}$ 920, Drees.

Choné, P., F. Evain, L. Wilner et E. Yilmaz (2014), «Réforme du financement des hôpitaux publics : quel impact sur leur niveau d'activité ?», Insee Analyses, $\mathrm{n}^{\circ} 15$.

CIS (2016), Antibiorésistance : un risque maîtrisable pour la santé humaine, animale, et pour l'environnement - 13 mesures pour maîtriser l'antibiorésistance, Comité interministériel pour la santé.

Clark, A. et C. Milcent (2011), «Public Employment and Political Pressure », Journal of Health Economics, vol. 30, pp. 1103-12.

Clark, A. et C. Milcent (2015), « Hospital Employment and Local Unemployment: Evidence from French Health Reforms? », Working Paper, Paris School of Economics, n² 2015-24.

CNAMTS (2016a), Améliorer la qualité du système de santé et maîtriser les dépenses Propositions de l'Assurance Maladie pour 2017, Caisse nationale de l'assurance maladie des travailleurs salariés.

CNAMTS (2016b), «Antibiotiques : les actions de l'assurance maladie pour promouvoir le bon usage », Rencontres santé publique - 7 Juin 2016, Caisse nationale de l'assurance maladie des travailleurs salariés.

Commission européenne (2013a), « Benchmarking Deployment of eHealth among General Practitioners », Report for the European Commission DG Communications Networks. Content \& Technology.

Commission européenne (2013b), «Environment: a fresh legal approach to improving air quality in Member States », Communiqué de presse, $\mathrm{n}^{\circ}$ 13-47.

Commission européenne (2014), « Patient Safety and the quality of care », Rapports Eurobaromètre spéciaux, $\mathrm{n}^{\circ} 411$.

Commission européenne (2017), «Infractions continues aux limites en matière de pollution atmosphérique : la Commission met en garde l'Allemagne, la France, l'Espagne, l'Italie et le Royaume-Uni », Communiqué de presse, $\mathrm{n}^{\circ}$ 17-238.

Compagnon, C. et A. Lopez (2015), « Pertinence et efficacité des outils de politique publique visant à favoriser l'observance », Rapport de l'IGAS, n 2015-037R.

Cour des comptes (2012), «Les arrêts de travail et les indemnités journalières versées au titre de la maladie », Rapport sur la sécurité sociale 2012, Paris.

Cour des comptes (2013), «Les accidents du travail et maladies professionnelles : les faiblesses de la politique de prévention du régime général de sécurité sociale », Rapport public annuel 2013, Paris. 


\section{ECO/WKP(2018)2}

Cour des comptes (2014a), «Les urgences hospitalières : une fréquentation croissante, une articulation avec la médecine de ville à repenser », Rapport sur l'application des lois de financement de la sécurité sociale 2014, Paris.

Cour des comptes (2014b), « La dette des établissements publics de santé », Paris.

Cour des comptes (2014c), «La diffusion des médicaments génériques : des résultats trop modestes, des coûts élevés », Rapport sur l'application des lois de financement de la sécurité sociale 2014, Paris.

Cour des comptes (2015), La Sécurité Sociale 2015, Rapport sur l'application des lois de financement de la sécurité sociale, Paris.

Cour des comptes (2016a), Simplifier la collecte des prélèvements versés par les entreprises, Paris.

Cour des comptes (2016b), L'hospitalisation à domicile, Paris.

Cour des comptes (2016c), Les données personnelles de santé gérées par l'assurance maladie : Une utilisation à développer, une sécurité à renforcer, Paris.

Cour des comptes (2016d), La Sécurité sociale 2016, Rapport sur l'application des lois de financement de la sécurité sociale, Paris.

Cour des comptes (2016e), La prévention des conflits d'intérêts en matière d'expertise sanitaire, Paris.

Cour des comptes (2016f), Les politiques de lutte contre les consommations nocives d'alcool, Paris.

Cour des comptes (2016g), Les finances publiques locales, Paris.

Cour des comptes (2016h), Les politiques publiques de lutte contre la pollution de l'air, Paris.

Coutrot, T. (2009), «Le rôle des comités d'hygiène, de sécurité et des conditions de travail en France », Travail et Emploi, ${ }^{\circ} 117$, pp. 25-38.

Coutrot, T. et E. Davie (2014), «Les conditions de travail en 2013 : une analyse par secteur du privé et versants de la fonction publique », Dares analyses, $\mathrm{n}^{\circ} 102$.

Cubaynes, M.-H., D. Noury, M. Dahan et E. Falip (2011), « Le circuit du médicament à l'hôpital », Rapport de l'Inspection générale des affaires sociales, Paris.

Dafny, L. (2005), « How do hospitals respond to price changes? », American Economic Review, vol. 95, ${ }^{\circ}$ 5, pp. 64-74.

Dahan, M. (2016), « Pilotage opérationnel du Plan d'action de promotion des médicaments génériques », Rapport de l'Inspection générale des affaires sociales, $\mathrm{n}^{\circ}$ 2015-89R.

Davezies, L. et L. Toulemon (2015), «Public reimbursement rates and health expenditures. A natural experiment », Annals of Economics and Statistics, n 119-120, pp. 179-197.

Davie, E. (2015), « Absence pour raisons de santé et lien avec les conditions de travail dans la fonction publique et le secteur privé », in Rapport annuel sur l'état de la fonction publique - Politiques et 
pratiques de ressources humaines - Faits et chiffres, Ministère de la décentralisation et de la fonction publique, pp. 217-51.

Delamaire, M. et G. Lafortune (2010), « Nurses in Advanced Roles: A Description and Evaluation of Experiences in 12 Developed Countries », OECD Health Working Papers, $\mathrm{n}^{\circ}$ 54, Éditions OCDE.

de la Maisonneuve, C. et J. Oliveira Martins (2013), «A Projection Method for Public Health and LongTerm Care Expenditures », Documents de travail du Département des affaires économiques de l'OCDE, n 1048 , Éditions OCDE.

de la Maisonneuve, C., R. Moreno-Serra, F. Murtin et J. Oliveira Martins (2016), «The drivers of public health spending: Integrating policies and institutions », Documents de travail du Département des affaires économiques de l'OCDE, $\mathrm{n}^{\circ} 1283$, Éditions OCDE.

Desprès, C., S. Guillaume et P.-E. Couralet (2009), «Le refus de soins à l'égard des bénéficiaires de la Couverture maladie universelle complémentaire à Paris », Rapport au Fonds de financement de la protection complémentaire de la couverture universelle du risque maladie.

Deumie, B., P. Georges et J.-P. Natali (2014), « Contrôle de l'Organisme gestionnaire du développement professionnel continu et évaluation du développement professionnel continu des professionnels de santé », Rapport de l'Inspection générale des affaires sociales.

DGCCRF (2016), Avantages commerciaux dans le secteur pharmaceutique, Direction générale de la concurrence, de la consommation et de la répression des fraudes, Paris.

DGOS (2013), Le recensement des activités de télémédecine, Direction générale de l'offre de soins. DGOS (2016), Atlas des SIH 2016, Direction générale de l'offre de soins.

Dormont, B. et M. Péron (2016), « Does Health Insurance Encourage the Rise in Medical Prices? A Test on Balance Billing in France », Health Economics, vol. 25, n 9, pp. 1073-89.

Drees (2015), L'état de santé de la population en France - Édition 2015, Drees.

Drees (2016a), La complémentaire santé : acteurs, bénéficiaires, garanties - Édition 2016, Drees. Drees (2016b), Portrait des professionnels de santé - Édition 2016, Drees.

Drees (2016c), Les dépenses de santé en 2015 - Résultats des comptes de la santé, Drees. Drees

(2016d), Les établissements de santé - Édition 2016, Drees.

Duval, J. et R. Lardellier (2012), «La redistribution verticale opérée par l'assurance maladie », Études et résultats, Drees, $\mathrm{n}^{\circ} 815$.

Eurostat (2016), « Agriculture biologique - La superficie des cultures biologiques est en hausse dans l'UE - Deux millions d'hectares supplémentaires depuis 2010 », Communiqué de presse, $n^{\circ} 208 / 2016$.

Fall, F., D. Bloch, P. Hoeller, J. Pareliussen et M. Pisu (2014), « Vulnerability of Social Institutions », OECD Economic Policy Papers, ${ }^{\circ} 11$, Éditions OCDE.

FFMPS (2017), «NMR - RA Quelles perspectives pour le financement du fonctionnement des MSP? », Journées Nationales de la FFMPS, 17 mars 2017. 
Garoche, B. (2016), «Les accidents du travail et les accidents de trajet », DARES Résultats, nº 039.

Geay, C. et G. de Lagasnerie (2013), « Projection des dépenses de santé à l'horizon 2060, le modèle PROMEDE », Documents de travail de la DG Trésor, $\mathrm{n}^{\circ} 8$.

Gobillon, L. et C. Milcent (2013), «Spatial Disparities in Hospital Performances », Journal of Economic Geography, vol. 13, n 6, pp. 1013-40.

Gobillon, L. et C. Milcent (2016), «Evaluating the Effect of Ownership Status on Hospital Quality: The Key Role of Innovative Procedures », Annals of Economics and Statistics, à paraître.

Golfouse, A. et B. Pheng (2015), «Les épreuves classantes nationales (ECN) donnant accès au 3ème cycle des études médicales », Rapport 2015 de l'Observatoire national de la démographie des professions de santé.

Guérin, S., A. Laplanche, A. Dunant et C. Hill (2013), « Mortalité attribuable à l'alcool en France en 2009 », Bulletin épidémiologique hebdomadaire (BEH), $\mathrm{n}^{\circ}$ 16-17-18, 7 mai, Institut national de veille sanitaire (InVS).

Guthmuller, S., F. Jusot, J. Wittwer et C. Desprès (2012), «Faire valoir ses droits à l'Aide complémentaire santé : les résultats d'une expérimentation sociale », Économie et statistique, n 455-456, pp. 53-70.

HCAAM (2013), Rapport Annuel 2013, Haut Conseil pour l'avenir de l'assurance maladie.

HCAAM (2014), La coopération entre professionnels de santé, Haut Conseil pour l'avenir de l'assurance maladie. HCFI-PS (2015), Rapport sur la lisibilité des prélèvements et l'architecture financière des régimes sociaux, Haut Conseil du financement de la protection sociale.

HCFI-PS (2017a), État des lieux actualisé du financement de la protection sociale - 11 janvier 2017, Haut Conseil du financement de la protection sociale.

HCFI-PS (2017b), Rapport sur la protection sociale des non salariés et son financement, Haut Conseil du financement de la protection sociale.

Husson, J.-F. et L. Aïchi (2015), «Rapport fait au nom de la commission d'enquête sur le coût économique et financier de la pollution de l'air », Rapport au Sénat, $\mathrm{n}^{\circ} 610$.

IGF (2013), Les professions réglementées, Inspection générale des finances.

Imai, Y., S. Jacobzone et P. Lenain (2000), « The Changing Health System in France », OECD Economics Department Working Papers, $\mathrm{n}^{\circ}$ 269, Éditions OCDE, Paris.

Jakoubovitch, S., M.-C. Bournot, É. Cercier et F. Tuffreau (2012), «Les emplois du temps des médecins généralistes », Études et résultats, $n^{\circ} 797$, Drees.

Jardin, M., A. Bocquier, S. Cortaredona, S. Nauleau, C. Million, S. Savard-Chambard, V. Allaria-Lapierre, V. Sciortino, G. Bouvenot et P. Verger (2012), «Prescriptions médicamenteuses potentiellement inadaptées chez les personnes âgées : une étude en Provence-Alpes-Côte d'Azur à partir des données de remboursements de l'assurance maladie », Revue d'épidémiologie et de santé publique, $\mathrm{n}^{\circ} 60$. 
Koechlin, F., P. Konijn, L. Lorenzoni et P. Schreyer (2014), «Comparing Hospital and Health Prices and Volumes Internationally : Results of a Eurostat/OECD Project », OECD Health Working Papers, $\mathrm{n}^{\circ}$ 75, Éditions OCDE, Paris.

Kunst, A., F. Groenhof, J. Mackenbach et Groupe de travail de l'Union européenne sur les inégalités socioéconomiques de santé (2000), «Inégalités sociales de mortalité prématurée : La France comparée aux autres pays européens », in Les inégalités sociales de santé, Paris.

Larochette, B. et J. Sanchez-Gonzalez (2015), « Cinquante ans de consommation alimentaire : une croissance modérée, mais de profonds changements », INSEE Première, $\mathrm{n}^{\circ} 1568$.

Larson, H., A. de Figueiredo, Z. Xiahong, W. Schulz, P. Verger, I. Johnston, A. Cook, et N. Jones (2016), « The state of vaccine confidence 2016: Global insights through a 67-country survey », EBioMedicine, $\mathrm{n}^{\circ} 12$, pp. 295-301.

Leduc, A. et A. Montaut (2016), «Tarification des complémentaires santé : déclin des solidarités dans les contrats individuels », Études et résultats, $\mathrm{n}^{\circ}$ 972, Drees.

Le Gouvernement (2016), Projet de loi de finances pour 2017 - Rapport économique social et financier, Paris.

Milcent, C. (2016a), «Premier bilan de la T2A sur la variabilité des coûts hospitaliers », Économie et prévision, à paraître.

Milcent, C. (2016b), «Up-coding and heterogeneity in hospitals' response: A Natural Experiment », Working paper $P S E, \mathrm{n}^{\circ} 201423$.

Ministère des Affaires sociales, de la Santé et des Droits des femmes (2015), Plan national d'action de promotion des médicaments génériques - mars 2015, Paris.

Mousquès, J. et F. Daniel (2015a), «Multi Multiprofessional Group Practices Generate Gains in Terms of Productivity and Expenditures professional Group Practices Generate Gains in Terms of Productivity and Expenditures », Questions d'économie de la santé, n 210, IRDES.

Mousquès, J. et F. Daniel (2015b), « The Impact of Multiprofessional Group Practices on the Quality of General Practice », Questions d'économie de la santé, n 211, IRDES.

OCDE (2000), OECD Economic Surveys France 2000, Éditions OCDE, Paris, http://dx.doi.org/10.1787/eco_surveys-fra-2000-en.

OCDE (2011a), Études économiques de l'OCDE : France 2011, Éditions OCDE, Paris, http://dx.doi.org/10.1787/eco_surveys-fra-2011-fr.

OCDE (2011b), Examens de l'OCDE des systèmes de santé : Suisse 2011, Éditions OCDE, Paris, http://dx.doi.org/10.1787/9789264120938-fr.

OCDE (2014a), Vieillissement et politiques de l'emploi : France 2014 : Mieux travailler avec l'âge, Éditions OCDE, Paris, http://dx.doi.org/10.1787/9789264206847-fr.

OCDE (2014b), Obesity Update, Éditions OCDE, Paris, www.oecd.org/health/healthsystems/obesity-update-previous-editions.htm. 
OCDE (2015a), Fiscal Sustainability of Health Systems, Bridging Health and Finance Perspectives, Éditions OCDE, Paris, http://dx.doi.org/10.1787/9789264233386-en.

OCDE (2015b), Panorama de la santé 2015, Les indicateurs de l'OCDE, Éditions OCDE, Paris, http://dx.doi.org/10.1787/health_glance-2015-fr.

OCDE (2015c), Lutter contre la consommation nocive d'alcool: Politiques économiques et de santé publique, Éditions OCDE, Paris, http://dx.doi.org/10.1787/9789264244580-fr.

OCDE (2016a), « Health policy in France », OECD Health Policy Overview, janvier, Éditions OCDE, www.oecd.org/france/Health-Policy-in-France-January-2016.pdf.

OCDE (2016b), Health Workforce Policies in OECD Countries: Right Jobs, Right Skills, Right Places, Éditions OCDE, Paris, http://dx.doi.org/10.1787/9789264239517-en.

OCDE (2016c), Better Ways to Pay for Health Care, Éditions OCDE, Paris, http://dx.doi.org/10.1787/9789264258211-en.

OCDE (2016d), Statistiques de l'OCDE sur la santé, Éditions OCDE, Paris, http://dx.doi.org/10.1787/health-data-fr.

OCDE (2016e), Examens environnementaux de l'OCDE : France 2016, Éditions OCDE, Paris, http://dx.doi.org/10.1787/9789264252592-fr.

OCDE (2017a), New health technologies: Managing access, value and sustainability, Éditions OCDE, Paris, http://dx.doi.org/10.1787/9789264266438-4-en.

OCDE (2017b), Behavioural Insights and Public Policy: Lessons from Around the World, Éditions OCDE, Paris, http://dx.doi.org/10.1787/9789264270480-en.

OCDE/UE (2016), Health at a Glance: Europe 2016: State of Health in the EU Cycle, Éditions OCDE, Paris, http://dx.doi.org/10.1787/9789264265592-en.

OMS (2016), Global Health Observatory data repository, Organisation mondiale de la santé, Genève.

ONDP (2014), ONDPS - Rapport 2013-14, Observatoire national de la démographie des professions de santé.

Ono, T., M. Schoenstein et J. Buchan (2014), «Geographic Imbalances in Doctor Supply and Policy Responses », Documents de travail de l'OCDE sur la santé, $\mathrm{n}^{\circ}$ 69, Éditions OCDE, Paris.

ONPV (2016), «L'offre de soins dans les quartiers prioritaires de la politique de la ville, en 2014 », Rapport annuel de l'Observatoire national de la politique de la ville 2015.

ONZUS (2015), Observatoire national des zones urbaines sensibles - Rapport 2014, Observatoire national des zones urbaines sensibles.

Paille, F. et M. Reynaud (2015), «L'alcool, une des toutes premières causes d'hospitalisation en France », Bulletin épidémiologique hebdomadaire (BEH), $\mathrm{n}^{\circ}$ 25-26, 7 juillet.

Paris, V., M. Devaux et L. Wei (2010), «Health Systems Institutional Characteristics: A Survey of 29 OECD Countries », Documents de travail de l'OCDE sur la santé, $n^{\circ}$ 50, Éditions OCDE, Paris. 
Paris, V., E. Hewlett, A. Auraaen, J. Alexa et L. Simon (2016), « Health care coverage in OECD countries in $2012 »$, Documents de travail de l'OCDE sur la santé, n 88, Éditions OCDE, Paris.

Persson, P. et M. Rossin-Slater (2016), «Family Ruptures, Stress, and the Mental Health of the Next Generation », American Economic Review, à paraître.

Pierron, L. (2016), «Complémentaire santé Sortir de l'incurie », Note Terra Nova, http://tnova.fr/notes/complementaire-sante-sortir-de-l-incurie.

Pisarik, J., T. Rochereau et N. Célant (2017), «État de santé des Français et facteurs de risque », Études et résultats, $\mathrm{n}^{\circ}$ 998, Drees.

PLFSS (2015), Projet de loi de financement de la sécurité sociale pour 2016, Ministère des Finances et des Comptes publics et Ministère des Affaires sociales, de la Santé et des Droits des femmes, Paris.

PLFSS (2016), Projet de loi de financement de la sécurité sociale pour 2017, Ministère des finances et des comptes publics et Ministère des Affaires sociales, de la Santé et des Droits des femmes, Paris.

Pollack, C. (2015), «L'effet du délai de carence sur le recours aux arrêts maladie des salariés du secteur privé », Dossiers Solidarité et Santé, n 58 , Drees.

Polton, D. (2015), « Rapport sur la réforme des modalités d'évaluation des médicaments », Rapport à la ministre des Affaires sociales, de la Santé et des Droits des femmes, Paris.

Premier Ministre (2011), Mise en cuvre de la Stratégie nationale de développement durable 20102013 : 1er rapport du Parlement-octobre 2011, Paris.

Ribassin-Majed, L. et C. Hill (2015), « Trends in tobacco-attributable mortality in France », The European Journal of Public Health, vol. 25, $\mathrm{n}^{\circ}$ 5, pp. 824-828.

Santé publique France (2016), Impacts de l'exposition chronique aux particules fines sur la mortalité en France continentale et analyse des gains en santé de plusieurs scénarios de réduction de la pollution atmosphérique.

Sassi, F., A. Belloni et C. Capobianco (2013), «The Role of Fiscal Policies in Health Promotion », Documents de travail de l'OCDE sur la santé, $\mathrm{n}^{\circ} 66$, Éditions OCDE, Paris.

Sécurité sociale (2014), Rapport de la commission instituée par l'article L. 176-2 du code de la sécurité sociale, Paris. Spilka, S., O. Le Nézet, M. Ngantcha et F. Beck (2015), «Les drogues à 17 ans : analyse de l'enquête ESCAPAD 2014 », Tendances, $\mathrm{n}^{\circ}$ 100, OFDT.

Véran, O. (2013), Hôpital cherche médecins, coûte que coûte, Rapport parlementaire.

Véran, O. (2016), Mission sur l'évolution du mode de financement des établissements de santérapport d'étape, Rapport parlementaire.

Vergier, N. (2016), « Accessibilité aux professionnels de santé libéraux : des disparités géographiques variables selon les conditions tarifaires », Études et résultats, $n^{\circ}$ 970, Drees. 